\title{
1-nm Resolution Functional Pattern Transfer to an Amorphous Elastomeric Material
}

\author{
Tyson C. Davis, ${ }^{\dagger}$ Jeremiah O. Bechtold ${ }^{\dagger}$ Anni Shi ${ }^{\dagger}$ Erin N. Lang, ${ }^{\dagger}$ Anamika Singh, ${ }^{\dagger}$ Shelley A. \\ Claridge* $*,+\neq$
}

†Department of Chemistry, Purdue University, West Lafayette, IN 47907

*Weldon School of Biomedical Engineering, Purdue University, West Lafayette, IN 47907

*Address correspondence to: claridge@purdue.edu, (phone) 765-494-6070

\begin{abstract}
Decades of work in surface science have established the ability to functionalize clean inorganic surfaces with sub-nm precision, but for many applications, it would be useful to provide similar control over the surface chemistry of amorphous materials such as elastomers. Here, we show that striped monolayers of diyne amphiphiles, assembled on graphite and photopolymerized, can be covalently transferred to polydimethylsiloxane (PDMS), an elastomer common in applications including microfluidics, soft robotics, wearable electronics, and cell culture. This process creates precision polymer films $<1 \mathrm{~nm}$ thick, with 1 -nm-wide functional patterns, that control interfacial wetting, reactivity, and adsorption of flexible, ultranarrow inorganic nanowires. The polydiacetylenes exhibit polarized fluorescence emission, revealing polymer location, orientation, and environment, and resist engulfment, a common problem in PDMS functionalization. These findings illustrate a route for controlling surface chemistry well below the length scale of heterogeneity in an amorphous material.
\end{abstract}

Extensive surface science research has demonstrated molecular- and even atomic-scale control over surface chemistry of metal and other inorganic surfaces, using carefully controlled environments and high-resolution characterization techniques such as scanning tunneling microscopy (STM) ${ }^{1,2}$ This approach has provided a basis for demonstrations of topological insulators and quantum computing, as well as illuminating molecular-scale details of polymerization (e.g. graphene nanoribbons, boronic aciddiols, polythiophenes, polydiacetylenes) ${ }^{3-6}$ and heterogeneously catalyzed reactions (e.g. Ullman coupling). ${ }^{7}$ The extremely high spatial resolution patterning achieved through classic surface science approaches would become even more powerful if it were also possible to leverage on flexible, technologically relevant amorphous materials (e.g. polymers, glasses), which lack the atomically precise lattice that often drives nm-resolution surface functionalization. ${ }^{8}$

Here, we report a straightforward route for 1-nm-resolution chemical patterning of an amorphous elastomeric material with a much larger mesh size (here, $\sim 10 \mathrm{~nm}$ ). This process is based on generation of a functional pattern on a flat, crystalline material, ${ }^{9-11}$ on-surface topochemical polymerization ${ }^{6,12}$ to stabilize the functional pattern, and subsequent covalent transfer to the amorphous material surface. Diacetylenes are convenient building blocks for functional patterning in this regard, since the relatively rigid polydiacetylene (PDA) backbone (persistence length $\sim 16 \mathrm{~nm})^{13,14}$ has the potential to retain significant ordering of pendant functional groups during transfer. Assembly in a striped phase, common in STM-characterized interfacial assembly, both creates nmresolution functional patterns and enables the exposed individual PDA backbones to be utilized in establishing covalent linkages to the amorphous material. Following transfer, optical properties of the PDA (including polarized fluorescence emission) enable us to characterize ordering in the transferred layer, overcoming otherwise significant challenges in characterizing chemical structure in soft, nonconductive, poorly ordered surfaces across the nm-to-mm scales relevant to chemical function.

Striped phases and related noncovalent functionalization chemistries represent an important but underutilized method for patterning interfaces at very high resolution for applications requiring structured adhesion, wetting, or assembly of nano- or microscale components. Molecules with long alkyl chains can lie down on 2D materials such as highly oriented pyrolytic graphite (HOPG), forming ordered molecular stripes ${ }^{9,11}$ stabilized by alkyl- $\pi$ interactions with the substrate and van der Waals interactions within the layer (Fig. 1a). Striped phases of certain functional diacetylenes (e.g. 10,12-pentacosadiynoic acid, PCDA) have been characterized previously by STM,6,15 illustrating alternating 1-nm stripes of headgroups and $\sim 5$-nm-wide stripes of exposed alkyl chains, as well as photopolymerization to form the conjugated PDA (Fig. 1a, right). Although such characterization is typically carried out at very small scales (image edge lengths $c a$. 10-100 nm), we have found previously that ordering can be extended to much larger scales (domains up to $10-100 \mu \mathrm{m}$ edge length), and can be characterized by scanning electron microscopy (SEM). ${ }^{16,17}$ We have also shown that striped phases comprised of nm-resolution patterns can be templated by microcontact printing ( $\mu \mathrm{CP})$, enabling hierarchical interface design with complex headgroup chemistries including carbohydrates. ${ }^{18}$ Moreover, topochemical polymerization of functional diacetylenes in 3D and 2D standing phase crystals ${ }^{19}$ has been extensively studied since the first reports by Wegner in $1969^{20}$, providing a large library of suitable compounds for surface functionalization. ${ }^{19,21}$ The response of functional PDA optical properties to environmental stimuli ( $\mathrm{pH}$, temperature, mechanical perturbations) is well known for standing phases, ${ }^{22}$ pointing to likely broad utility for striped phase PDA films. Chemical patterns embedded in striped phases can also exhibit strong 
directing effects in further assembly at the interface - recently, we have demonstrated that 1-nm-wide patterns of headgroup dipoles in striped phases on HOPG direct assembly of flexible, ultranarrow gold nanowires (AuNWs) with high precision. ${ }^{23}$

In this work, we show that striped PDA films on HOPG can be covalently transferred to polydimethylsiloxane (PDMS), an elastomer common in wearable electronics, ${ }^{24}$ microfluidics, ${ }^{25}$ cell culture, ${ }^{26}$ and soft robotics, ${ }^{27}$ using the hydrosilylation reaction that is the basis for the PDMS curing process. This polydiacetylene-on-amorphous material transfer (PATRN) process creates 1-nm-wide functional patterns on the PDMS surface, which generate spatially resolved interactions with the environment (assembly of long, flexible inorganic nanowires, wetting with solvents, interactions with ions), and resist engulfment, a common problem with surface functionalization of PDMS. 28
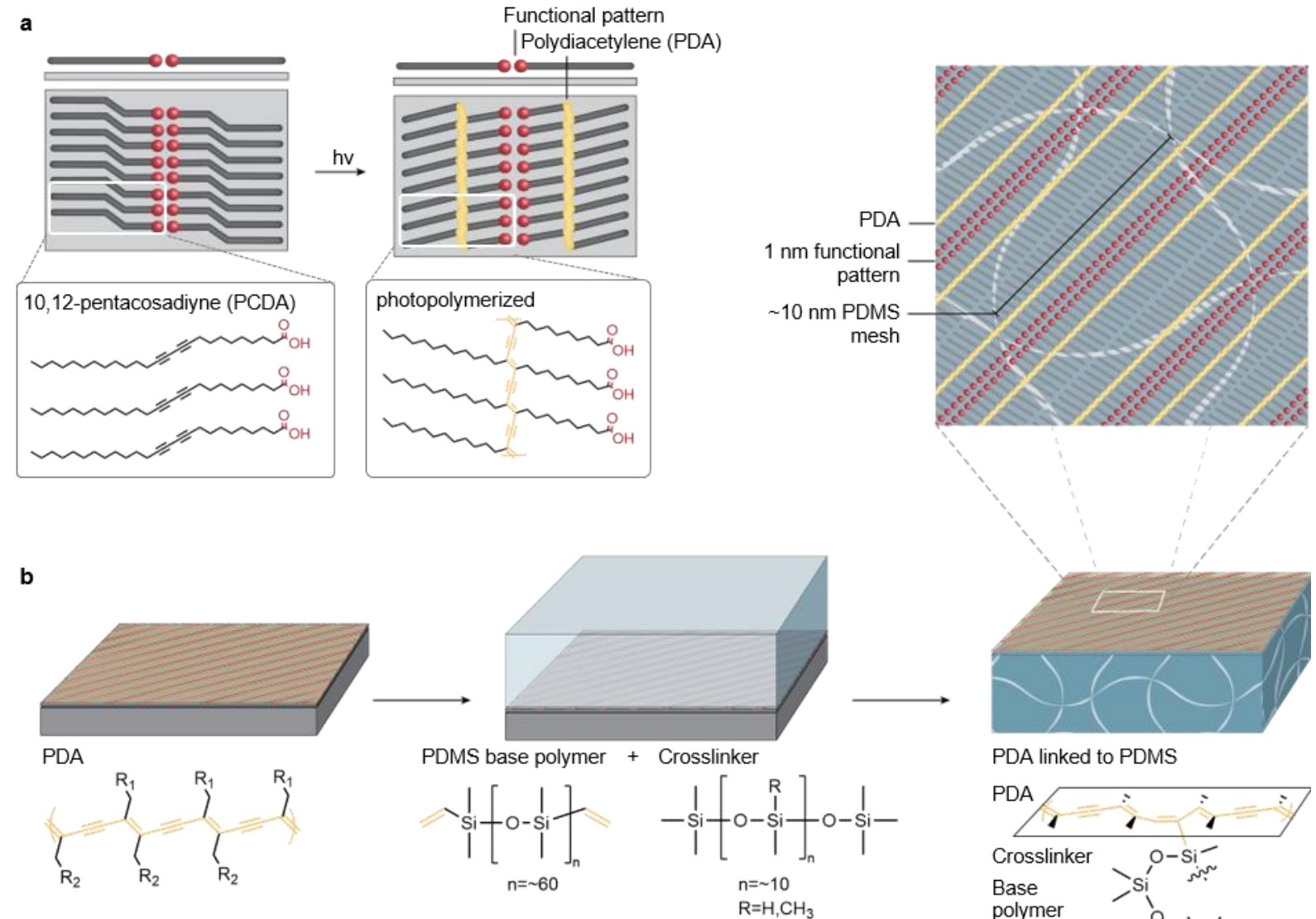

PDA linked to PDMS

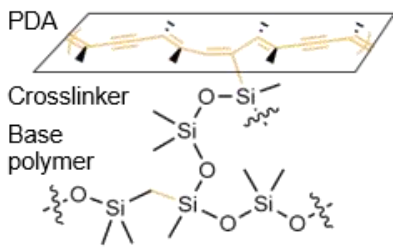

Figure 1. (a) Illustration of diyne amphiphile assembly on HOPG and photopolymerization, forming 1-nm-wide functional patterns. (b) Illustration of hydrosilylation reaction used to exfoliate striped diyne monolayer onto amorphous PDMS elastomer.

\section{RESULTS AND DISCUSSION}

Preparation and transfer of striped monolayers on HOPG. Striped monolayers of 10,12-pentacosadiynoic acid (PCDA) and 1,2-bis(10,12-tricosadiynoyl)-sn-glycero-3-phosphoethanolamine (dPE) were prepared via Langmuir-Schaefer (LS) conversion 16,29 33 or by $\mu \mathrm{CP}$, and polymerized via UV irradiation (Fig. 2). In each monolayer, the lamellar axis extends along the direction of the PDA backbone (highlighted in gold in Fig. 2a). Paired headgroups (COOH for PCDA and phosphoethanolamine for dPE) along the center of each stripe generate functional patterns $\sim 1 \mathrm{~nm}$ wide. In AFM images (Fig. 2b), striped lamellar patterns are visible, with periodicities (6.5 nm for PCDA and $6.1 \mathrm{~nm}$ for dPE) commensurate with those in the models. SEM images of PCDA (Fig. 2c) illustrate lamellar orientation, visible based on cracks formed in the monolayer under the electron beam. ${ }^{34}$ Domains are visible as brighter regions (stronger electron scattering) against a darker background representing unfunctionalized areas of the HOPG substrate. Larger-scale SEM images (Fig. 2c, right, and 2d) illustrate domain structure at scales similar to those in fluorescence images in figures below. For some experiments presented here, we utilized $\mu \mathrm{CP}$ square of striped phases to achieve geometric control over surface patterning (Fig. 2d). In others, monolayers were assembled by LS conversion, which produces longer-range molecular domain ordering. For LS conversion, we often chose conditions that produce large oval vacancies, ${ }^{34,35}$ similar to Fig. $2 c$ (right), providing a convenient internal standard to distinguish optical and other properties of functionalized and unfunctionalized areas of the PDMS. See Supplementary Figs. 1-3 for larger AFM and SEM images of PCDA and dPE. 


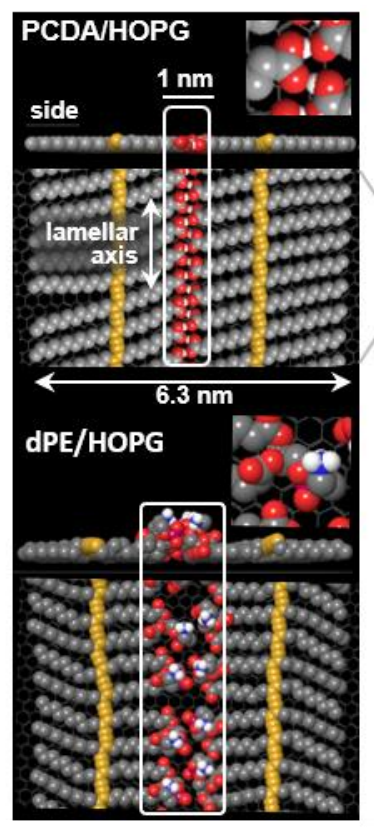

b
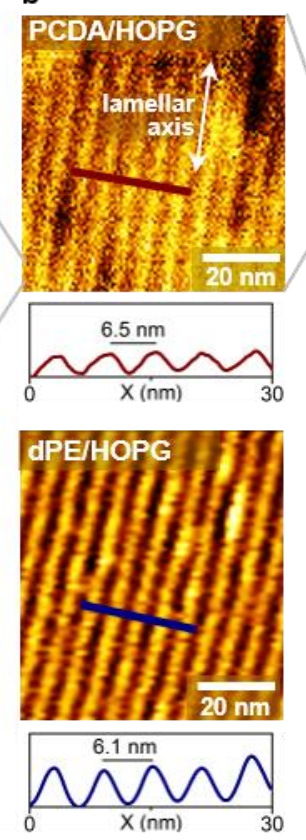

C
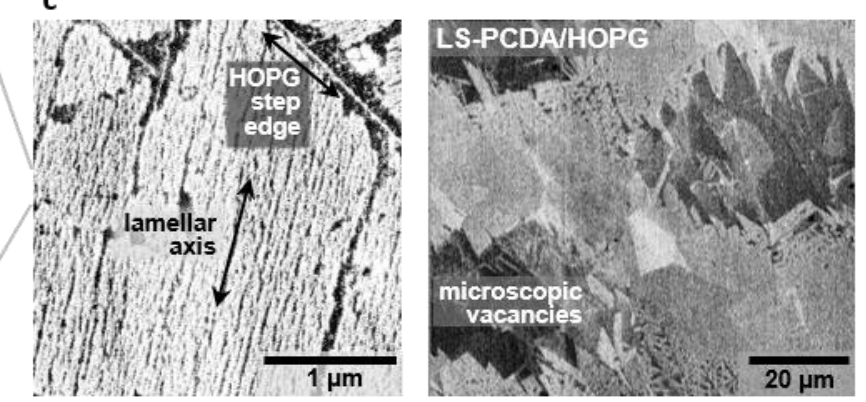

d
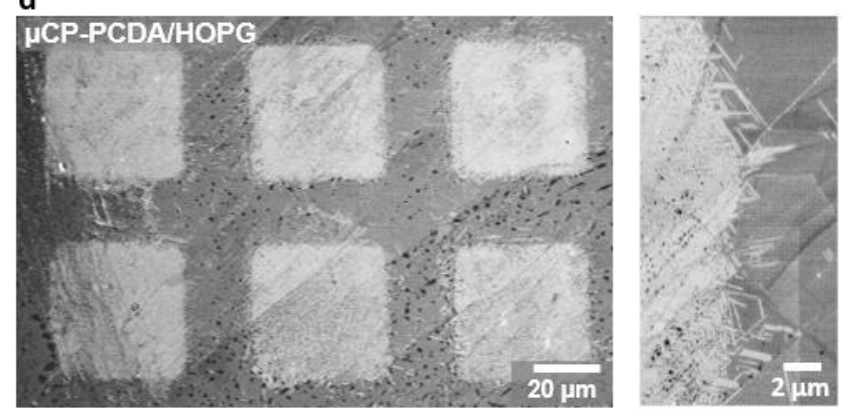

Figure 2. (a) Molecular models of photopolymerized monolayers of PCDA (top) and dPE (bottom) on HOPG, illustrating lamellar structure and periodicity. (b) AFM images of PCDA (top) and dPE (bottom) on HOPG, illustrating lamellar structure. (c) SEM images illustrating domain structure of PCDA at scale at which lamellar axis is still visible (left) and at larger scale (right). (d) SEM images of PCDA patterned on HOPG by microcontact printing $(\mu \mathrm{CP})$. Right image shows an enlargement of the edge of a $\mu \mathrm{CP}$-patterned square. Supplementary Figs. 1 and 2 show large SEM images for both PCDA/HOPG and dPE/HOPG.

Comparisons of molecular film structure after transfer to PDMS. Polydimethylsiloxane (PDMS, commercially available SYLGARD-184) was then cast on the monolayers and cured to allow Pt-catalyzed hydrosilylation to occur between Si-H bonds in PDMS crosslinker and C-C multiple bonds (vinyl groups in PDMS base polymer and PDA backbones in monolayer); literature reports $^{36}$ and reaction of structurally similar model compounds in solution (see Supplementary Fig. 4) support reactivity of conjugated alkynes toward hydrosilylation. Molecular models of dPE striped phases (Fig. $3 \mathrm{~b}$ ) illustrate that formation of new covalent bonds produces torsion along the PDA backbone. Following crosslinking, the PDMS and transferred PDA polymer layer were gently removed from the HOPG substrate. Under the curing conditions utilized here, bare PDMS forms a crosslinked network with a mesh size of $9.9 \pm 4.4 \mathrm{~nm}$ (Fig. 3a, left, and Supplementary Fig. 5). In contrast, PDMS surface-functionalized with PCDA exhibits linear features (>100 nm in Fig. 3a, right), consistent with transferred PDA polymers extended along the surface. Microscopic features are consistent with molecular domain structures observed on HOPG (Fig. 3a, far right, and Supplementary Fig. 6).

Scanning probe characterization of PATRN-generated monolayers on PDMS is substantially more challenging than on the highly structured HOPG substrate; the lack of electronic conductivity of the PDMS additionally precludes monolayer characterization by SEM. However, optical properties of the PDA backbone have been extensively characterized in 3D crystals and standing-phase films, ${ }^{37-39}$ and provide a useful means of characterizing PATRN monolayers of PDAs on PDMS. Typically 3 to 20 standing-phase layers of alkyl PDAs are required for detection based on optical absorbance, preventing observation of red and blue colors commonly used to characterize PDA standing phases in transmission. ${ }^{40}$ However, PATRN films on PDMS exhibit fluorescence emission (Fig. 3c and Supplementary Figs. 7-9). Fluorescence spectra (Fig. 3d) are distinct from those of bare PDMS (Supplementary Fig. 10) and reveal peaks with shapes consistent with those observed previously for PDA emission in standing phases (main excitonic peak + vibrational sidebands offset by $990 \mathrm{~cm}^{-1}$ (C-C stretch), $1480 \mathrm{~cm}^{-1}$ (C=C stretch), and $2100 \mathrm{~cm}^{-1}$ (CEC stretch)), 41,42 though the main excitonic peak is blue-shifted (to $546-549 \mathrm{~nm}$ ) in comparison with bulk red-form PDAs ( $\sim 70 \mathrm{~nm}) .{ }^{39}$ Since previous studies involve standingphase films, in which each PDA is flanked by either PDAs or monomeric diacetylenes that may act to stabilize the excited state, the blue shift observed here could reasonably result from isolation of individual PDAs on the PDMS surface. dPE monolayer fluorescence intensity is approximately 10x that of PCDA (Fig. 3e; insets show representative image regions captured using equivalent microscope settings), possibly consistent with increased PDA torsion in poly-dPE (model, Fig. 2a) due to geometric constraints imposed by the dual-chain molecular architecture. Fluorescence microscopy of PCDA or dPE on HOPG does not produce observable molecular domain features (Supplementary Fig. S10), consistent with quenching of the PDA by the HOPG substrate, and/or formation of the highly ordered, non-fluorescent blue form of the PDA. ${ }^{38,39}$ Previous work has indicated that the PDA exciton delocalizes over $\sim 6$ repeat units $(\sim 3 \mathrm{~nm}),{ }^{43}$ and it has been argued in the context of previous work on standing-phase PDA films ${ }^{44}$ that as long as PDA twisting (in the formation of red or other phases) does not further confine the exciton, the spectrum would remain similar. Here, that would appear to imply that the average distance between PDA/PDMS crosslinks is greater than 6 repeat units, which would be reasonable, given the $\sim 10-\mathrm{nm}$ mesh size of the PDMS. Changes in PDMS base:crosslinker ratio modulated fluorescence intensity, but not peak wavelengths (Supplementary Figs. 11-14). 
Differences in local fluorescence intensity can be utilized to distinguish between chemically different areas in a transferred multicomponent PATRN film. Langmuir films of mixtures of PCDA and dPE produce phase-segregated striped phases on HOPG (Fig. 3f, top); AFM images show clear differences between rows of dPE (bright stripes) and PCDA (dark background). SEM images (Fig. 3f, middle) exhibit contrast between the two molecules at microscopic scales; bright (highly scattering) domains correspond to dPE. After exfoliation onto PDMS, monolayer regions of with differing fluorescence emission intensities are visible (Fig. 3f, bottom, and Supplementary Fig. 15).

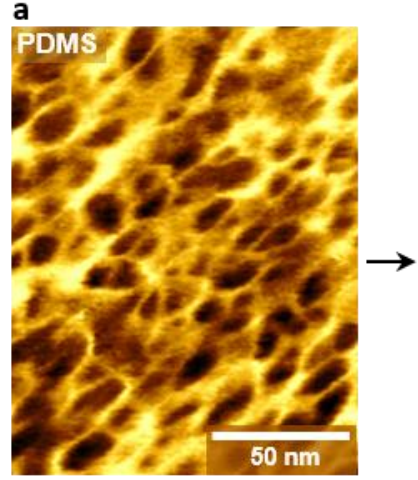

C
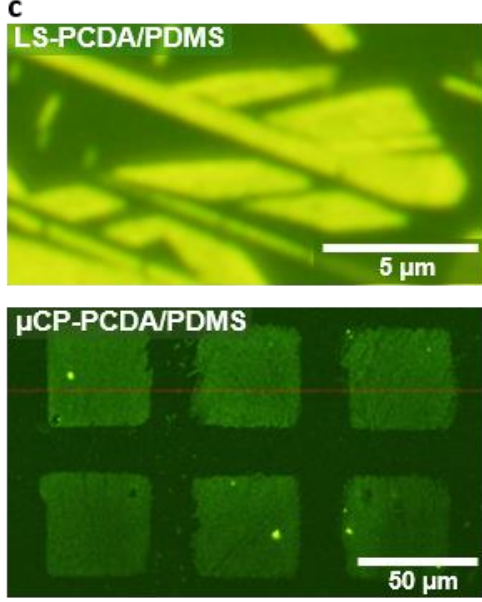

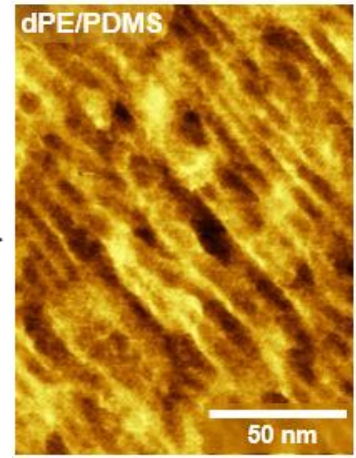

d

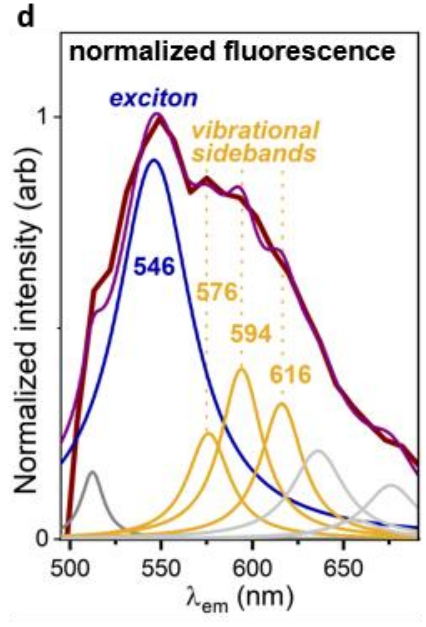

b

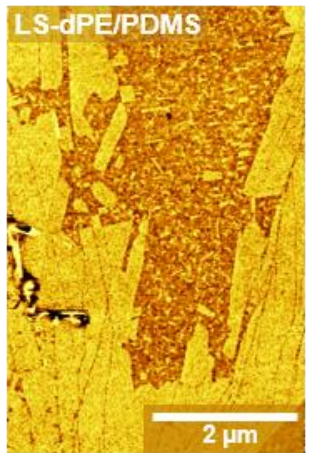

e

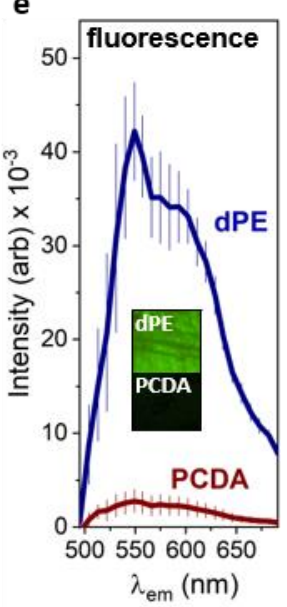

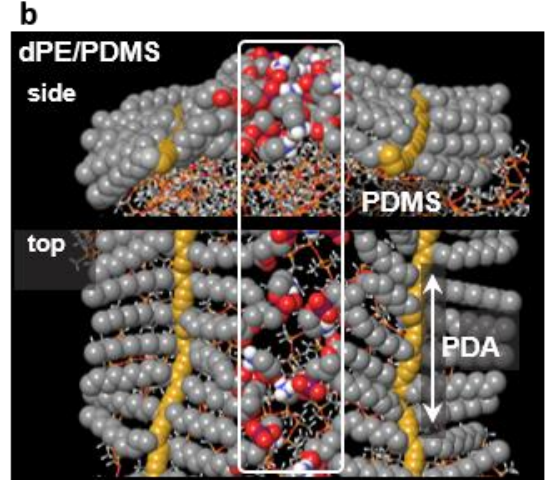

f

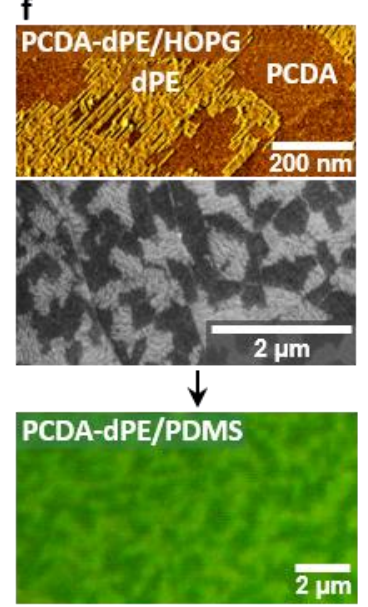

Figure 3. (a) AFM images illustrating lamellar structure in dPE transferred to PDMS. (b) Molecular model illustrating polymerized dPE/PDMS. (c) Fluorescence micrographs of PCDA/PDMS, illustrating large domains with hexagonal symmetry originally assembled on HOPG by LS transfer (top), and $\mu$ CP square patterns (bottom). (d) Normalized fluorescence spectrum for PCDA, with peak fitting for PDA exciton and vibrational sidebands. (e) Fluorescence spectra of PCDA (red trace) and dPE (blue trace) on PDMS, illustrating difference in emission intensity. (f) AFM phase (top) and SEM (middle) images showing a mixed monolayer of PCDA-dPE/HOPG. (f,bottom) Fluorescence micrograph of PCDA-dPE/PDMS, illustrating local variations in fluorescence.

Identifying molecular orientation in transferred molecular films. Identifying the orientation of 1-nm-wide linear features in PATRN films is also possible using polarized fluorescence imaging. Previously, others have demonstrated in highly oriented bulk crystals that PDAs exhibit a polarized free exciton ${ }^{1} \mathrm{~B}_{\mathrm{u}}$ transition, with $\alpha_{\|} / \alpha_{\perp}=600.45$ Here, we illustrate that this property can be utilized to identify the orientation of functional patterns in PATRN films. To maximize visibility, we utilized molecular layers assembled on $\mathrm{MoS}_{2}$, which previously we have reported produces sets of long, narrow domains with lengths of several $\mu$ m. ${ }^{46}$ Here, when transferred to PDMS, these domain structures create linear features in fluorescence images, with well-defined molecular orientation over scales easy to visualize in fluorescence. Fig. 4a (top) shows an unpolarized fluorescence image of dPE/PDMS, with the lamellar axes in two areas highlighted with arrows. When the emission channel is polarized (orientation illustrated by arrow in upper left corner), emission is visible from domains aligned with the polarization direction. For larger SEM images of monolayers prior to transfer, and polarized emission images, see Supplementary Figs. 16-20. 
a
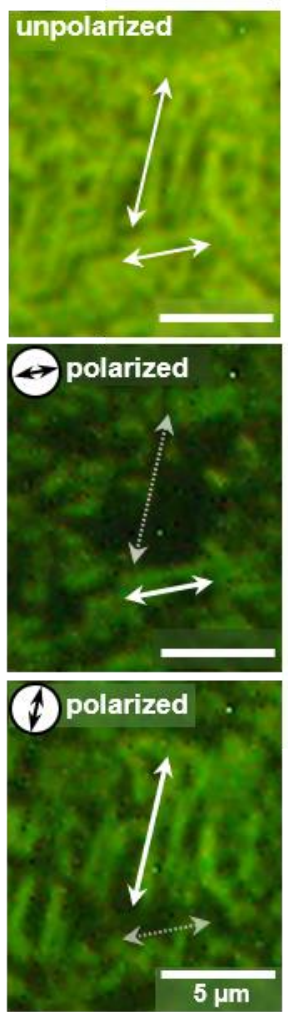

b
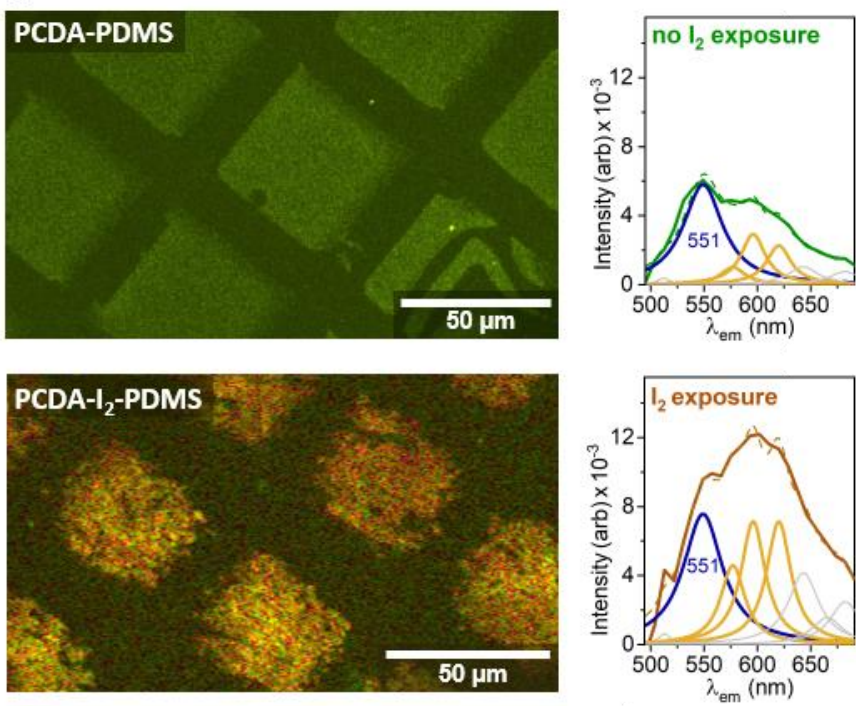

c
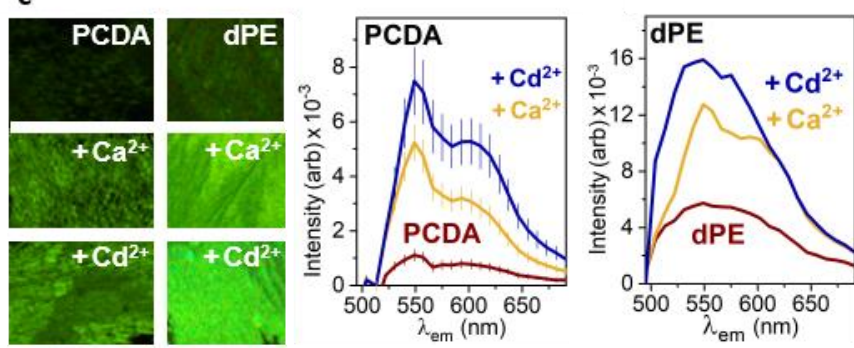

Figure 4. (a) Unpolarized (top) and polarized (middle, bottom) fluorescence images of the same area of dPE/PDMS, with emission channel polarized along direction indicated by arrow in top left corner. A pair of white arrows in center of each image guide the eye to areas with long-range molecular alignment on the two polarization axes. (b) Fluorescence images of PCDA monolayers assembled on HOPG by $\mu \mathrm{CP}$, polymerized, and (top) transferred to PDMS, or (middle) exposed to $\mathrm{I}_{2}$ (aq), and subsequently transferred to PDMS. (right) Fluorescence spectra of unmodified and $\mathrm{I}_{2}$-exposed $\mu \mathrm{CP}$-PCDA/PDMS, with Lorenzian peak fits illustrating fluorescence intensity increase in range of wavelengths associated with vibrational sidebands. (c) Fluorescence images of PCDA (left column) and dPE (right column) on PDMS, as-transferred (top), and in the presence of $100 \mathrm{mM} \mathrm{Ca}^{2+}$ (middle) or Cd2+ (bottom). (right) Fluorescence spectra of PCDA and dPE on PDMS, as-transferred (red trace), and with $100 \mathrm{mM} \mathrm{Ca}^{2+}$ buffer (gold trace) or Cd ${ }^{2+}$ buffer (blue trace) illustrating increase in fluorescence intensity.

Chemical manipulation of PDA backbones and headgroups to alter emission. Previously, it has been demonstrated that exposure of PDA molecular wires to $\mathrm{I}_{2}$ increases conductivity up to 3 orders of magnitude, 47 and can convert blue-form PDA to the red form. 47,48 Thus, I2 exposure provides a potential path to chemically modulate optical properties of PATRN films to distinguish between patterned areas. Fig. $4 \mathrm{~b}$ illustrates the effect of $\mathrm{I}_{2}$ on the fluorescence of PCDA transferred to PDMS. Square patterns of PCDA striped phases deposited on HOPG by microcontact printing, and subsequently transferred to PDMS, appear green in fluorescence images (Fig. 4b, top). Exposure to I2 prior to transfer to PDMS results in a visible shift in fluorescence emission (Fig. 4b, bottom), so that the square pattern appears orange, with a substantial increase in emission throughout the range of wavelengths associated with vibrational sidebands. See Supplementary Figs. 21 and 22 for fluorescence spectrum of iodine in solution, and fluorescence images of LS-PCDA/PDMS+I2.

Previously, others have demonstrated that standing phase molecular layers of polydiacetylenes (e.g. PCDA) can be utilized to sense ions that interact with headgroups..$^{22}$ Typically, such absorbance measurements require at least 3 layers of standing phase. ${ }^{49}$ We observe that interactions with buffers containing divalent ions (here, $100 \mathrm{mM} \mathrm{Ca}^{2+}$ or $\mathrm{Cd}^{2+}$ ) increase fluorescence emission (Fig. 4c, left) for transferred monolayers of PCDA and dPE. Fluorescence emission spectra (Fig. 4c, right) of PCDA/PDMS and dPE/PDMS exposed to divalent ions illustrate 5 -fold and 3-fold increases in overall fluorescence intensity for PCDA and dPE exposed to Ca ${ }^{2+}$ and somewhat greater increases (7-fold and 4-fold, respectively), for exposure to $\mathrm{Cd}^{2+}$. Previous studies of standing phases have associated interactions with divalent ions with a transition to the blue form of PDA, ${ }^{22}$ which is non-emissive; here, the increase in fluorescence intensity may indicate that for striped phases, the combination of covalent bonds to the PDMS elastomer and interactions between headgroups operate together to increase torsion on (and thus emission from) the PDA backbone. See Supplementary Figs. 23-25 for larger fluorescence images. 
Use of surface-patterned PDMS. The 1-nm-wide features in PATRN films can be used to direct assembly of objects including nanowires on the amorphous surface. Recently, we demonstrated that 1-nm-wide dipole arrays created by dPE/HOPG can be used to orient and order ultranarrow gold nanowires (AuNWs) with metal core diameters $1.5-2 \mathrm{~nm}$, and lengths up to $\sim 1 \mu \mathrm{m} .{ }^{23}$ In solution (cyclohexane solvent), the narrow, flexible AuNWs form bundles due to to depletion attraction interactions between their oleylamine (OM) ligand shells. However, when exposed to dPE/HOPG, bundles of wires adsorb, separate, and straighten, which appears to be due to repulsive interactions between AuNWs that undergo transverse polarization when exposed to arrays of dPE headgroup dipoles (see Supplementary Fig. 26 for more detailed discussion).

a

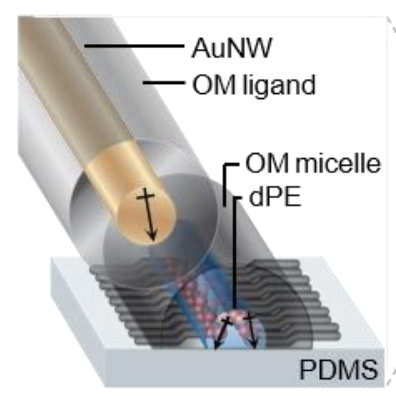

b

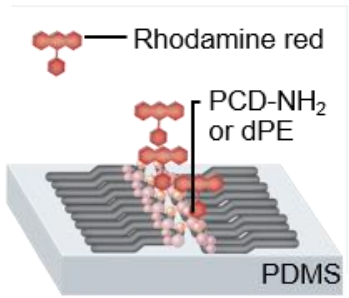

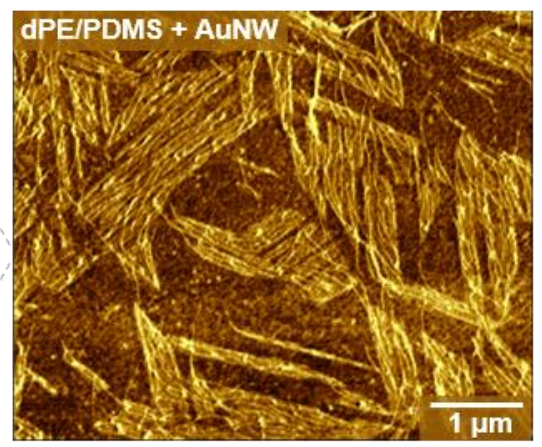

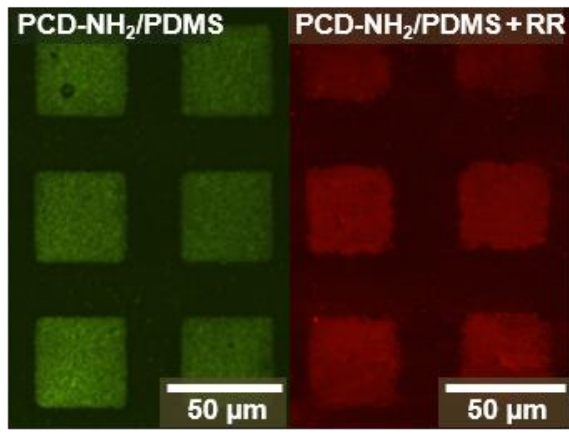

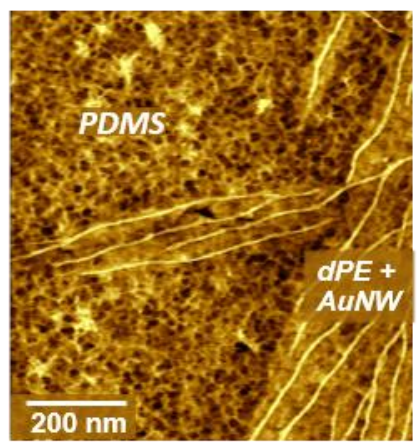

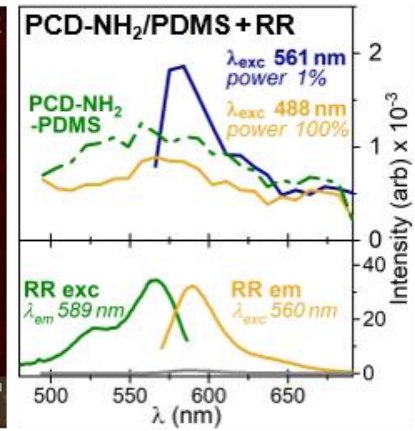

C

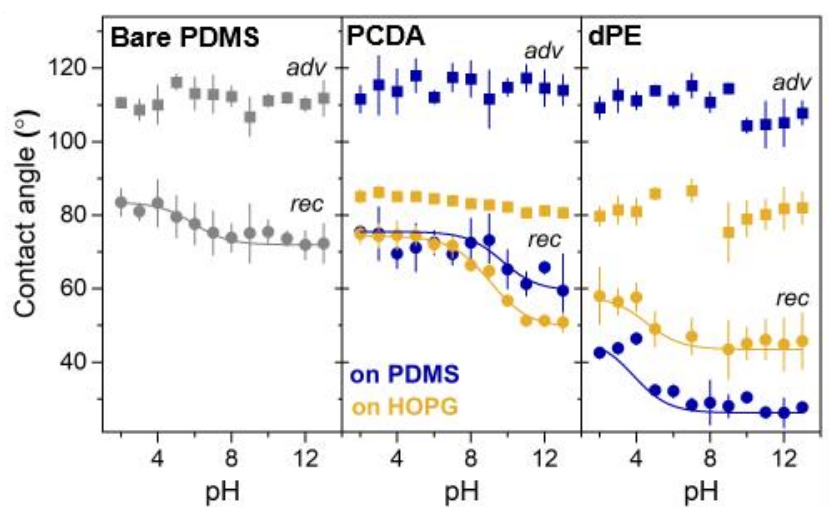

Figure 5. (a) Cartoon of AuNW adsorption to dPE/PDMS, and AFM images illustrating AuNW adsorption on dPE/PDMS in an area with several narrow domains. (b) (left) Cartoon of RR reaction with striped phases displaying primary amines. (center) Fluorescence images of $\mu \mathrm{CP}-\mathrm{PCD}-\mathrm{NH}_{2}$ /PDMS before (left) and after (right) reaction with RR NHS ester and subsequent vigorous washing. (right) Fluorescence spectra of unmodified (green dashed) and RR-modified (blue, orange) PCD-NH2/PDMS films. (c) Contact angle titrations for bare PDMS (left), PCDA (center), and dPE (right). For PCDA and dPE, contact angle titration are shown on HOPG (gold) and on PDMS (blue) for comparison.

To demonstrate that the 1-nm-wide functional patterns on PDMS can also be utilized to control ordering of nanoscale components at the interface, we exposed dPE-PDMS surfaces to similar solutions of AuNWs (Fig. 5a). AuNWs adsorb selectively in regions of the surface functionalized with $\mathrm{dPE}$, align with the molecular domain axis, and separate, much as they do on dPE/HOPG. Average centerto-center distances for parallel AuNWs are $21 \pm 4 \mathrm{~nm}$, slightly less than the Bjerrum length for cyclohexane. See Supplementary Fig. 27 for larger AFM images comparing adsorption on bare HOPG, dPE/HOPG, PDMS, and dPE/PDMS.

Rows of primary amines in $\mathrm{dPE}$ or in simple amines such as 10,12-pentacosadiynamine (PCD- $\mathrm{NH}_{2}$ ) can serve as functional handles for further coupling reactions, enabling the chemistry of PATRN films to be customized based on application (Fig. 5b). To assess headgroup accessibility and reactivity, squares of PCD-NH2 striped phases were prepared on HOPG by $\mu \mathrm{CP}$ and transferred to PDMS 
(see Supplementary Figs. 28-29 for larger fluorescence images and similar experiments conducted with dPE monolayers). Following covalent transfer of the PCD-NH 2 monolayers to PDMS, substrates were immersed in a reaction mixture containing Rhodamine red (RR) NHS ester, and allowed to react for $24 \mathrm{~h}$. To remove physisorbed RR, PCD-NH$/ \mathrm{PDMS}+\mathrm{RR}$ substrates were then subjected to rigorous cleaning (see Experimental Methods). Fig. 5b (center) shows a PCD- $\mathrm{NH}_{2} / \mathrm{PDMS}+\mathrm{RR}$ surface following cleaning, illustrating that fluorescence is localized to areas of PCD- $\mathrm{NH}_{2}$ surface functionalization. Emission spectra of PCD-NH2/PDMS+RR (blue trace), illustrate the presence of a strong peak at the RR emission maximum when the surface is excited at $561 \mathrm{~nm}$, near the RR excitation maximum.

PDMS is frequently utilized for microfluidics, due to its straightforward moldability. However, the unmodified surface is fairly hydrophobic, raising complications in some applications. Further, due to the elastomeric structure, hydrophilic surface modifications (e.g. plasma treatment) are typically engulfed within hours to present a lower energy surface.50 Previously, we have performed contact angle titrations of striped monolayers on HOPG, which reveal that the relatively nonpolar environment at the interface increases $\mathrm{pK}_{1 / 2}$ values for functional groups directly adjacent to the interface (e.g. the $\mathrm{pK}_{1 / 2}$ of the COOH groups in a PCDA monolayer on HOPG is $\sim 9 .{ }^{29}$ In Fig. 5a (center), this is visible as the midpoint of the sigmoidal curve of the receding contact angle (lower yellow trace). After transfer to PDMS, advancing angles of both PCDA/PDMS (center panel, top blue trace) and dPE/PDMS (right panel, top blue trace) are very similar to the advancing contact angles of bare PDMS (left panel, top grey trace), which may suggest that in the absence of a liquid layer at the interface, the hydrophilic headgroups are buried to minimize surface energy. However, receding angles for both PCDA/PDMS and dPE/PDMS (lower blue traces in middle and right panels, respectively) are lower, particularly for $\mathrm{dPE} / \mathrm{PDMS}$ and express pH-dependent differences in wettability similar to those observed for PCDA/HOPG and dPE/HOPG. These observations would be consistent with hydrophilic headgroups contributing to the de-wetting process. Receding contact angles remain at similar levels over up to 46 days (Supplementary Fig. 30), indicating that the functional patterns remain available at the interface for time periods greatly exceeding engulfment timescales for monomeric functionalization chemistries.

\section{CONCLUSIONS}

Here, we have demonstrated the capability to assemble 1-nm-wide functional patterns on a flat, crystalline surface, covalently link structural elements of the pattern through polymerization, and subsequently transfer the patterns to the surface of a porous amorphous material. As a result, surface functionalization can achieve ordering well below the length scale of heterogeneity of the bulk material. Optical properties of the PDA backbone make it reasonably straightforward to assess the location, orientation of elements of the surface pattern, as well as chemical interactions with the environment. We demonstrate that the transferred 1-nmresolution patterns can be utilized to control adsorption and ordering of AuNWs with diameters $<2 \mathrm{~nm}$ and lengths up to $1 \mu \mathrm{m}$, and can undergo further reactions to elaborate the monolayer chemistry. The strategy we describe utilizes members of a broad class of functional building blocks, and would be easily extended to other classes of surface functional patterns, and other amorphous materials capable of undergoing reactions with chemistry available in either the PDA backbone or the functional headgroups.

\section{MATERIALS AND METHODS}

Preparation of striped monolayers on HOPG. Unless otherwise stated, PCDA and other striped monolayers were prepared using a previously reported ${ }^{34}$ LS conversion method, or a previously reported $\mu \mathrm{CP}$ procedure for striped phase assembly. ${ }^{18} \mathrm{~A}$ typical preparation via LS conversion is described briefly here; see Supplementary Information for additional procedures. LS conversion was performed using a MicroTrough XL Langmuir-Blodgett trough (Kibron Inc., Helsinki, Finland). PCDA was assembled at the airwater interface by depositing $30 \mu \mathrm{L}$ of a $0.5 \mathrm{mg} / \mathrm{mL}$ solution of PCDA in chloroform on a subphase of Milli-Q water ( 18 M $\Omega \cdot \mathrm{cm})$ at $30^{\circ} \mathrm{C}$. The chloroform was allowed to evaporate for $15 \mathrm{~min}$ before the trough barriers were slowly moved inward (8.5 $\left.\mathrm{mm} / \mathrm{min}\right)$ until the target mean molecular area of $30 \AA^{2} /$ molecule was reached. A freshly cleaved HOPG substrate was mounted on an automated dipper attachment, ${ }^{16}$ with the HOPG nearly parallel to the air-water interface (cleaved side facing down), then lowered horizontally at a rate of $6 \mathrm{~mm} / \mathrm{min}$. After $2 \mathrm{~min}$ of contact with the interface, the HOPG was gently lifted out of contact with the liquid at the same speed, and blown dry with UHP N2. Monolayers were then polymerized under a hand-held UV lamp ( $254 \mathrm{~nm}, 8 \mathrm{~W}$ ) for 1 $\mathrm{h}$ with $\sim 2 \mathrm{~cm}$ between the lamp and the substrate.

Covalent transfer of polymerized diyne amphiphile striped phases from HOPG to PDMS. Transfer of polymerized diyne amphiphile striped phases was typically performed using SYLGARD 184 silicone elastomer base and curing (crosslinking) agent, at a 10:1 (m/m) ratio. The mixture was stirred for ca. $5 \mathrm{~min}$, then poured onto a functionalized HOPG substrate, and deaerated in a vacuum desiccator until no bubbles remained. The PDMS was cured for $24 \mathrm{~h}$ at $60{ }^{\circ} \mathrm{C}$, also allowing the PDMS to react with the polymerized monolayer. The cured (solid) PDMS was then peeled away from the HOPG substrate, resulting in transfer of the polymerized diyne amphiphile film to the free PDMS.

Wide-field fluorescence microscopy. Wide-field fluorescence micrographs were obtained using an 0lympus BX-51 optical microscope with an Olympus DP71 color camera. Images were acquired using either a 40X (metallographic, plan-fluorite aberration correction, NA $=0.75$, infinity corrected optics), or a 100X (metallographic, apochromatic and flat field correction, NA $=0.95$ ) brightfield objective. A UMWB2 filter cube was utilized with an 460-490 nm excitation band-pass filter, a dichroic filter wavelength of $500 \mathrm{~nm}$ and a long-pass emission filter wavelength of $520 \mathrm{~nm}$. Typical image collection times were $60 \mathrm{~s}$ with a resolution of 1024 $\times 1024$ pixels. For polarized emission images, an Olympus U-AN360P 360-degree rotatable analyzer with a minimum angle of 0.1 degree was placed in the emission path between the filter cube and the camera. 
Confocal luorescence microscopy and spectral imaging. Confocal micrographs and associated spectra were acquired utilizing a Zeiss LSM 880, Axio Examiner upright confocal microscope. Unless otherwise stated in the manuscript, excitation was provided by a $488 \mathrm{~nm}$ Ar laser set to $100 \%$ power. Data were obtained using a 20X objective (plan-apochromatic, dry, NA = 0.80), with a 0.17$\mathrm{mm}$ cover glass placed on the sample, and a 32-channel GaAsP spectral photomultiplier tube detector. Micrographs and corresponding spectra were obtained at a resolution of 2856 pixels per line, a bit depth of 16 bits, a dwell time of $11.75 \mu$ s/pixel (using unidirectional scanning and averaging 16 times per line), and a pinhole set to 1 Airy unit. For detection, 23 of the 32 channels were used to collect data, with bins centered at values from 495-691 nm and a resolution (bin width) of 8.9 nm. Bare PDMS spectra were subtracted from monolayer spectra during processing to minimize contributions from PDMS autofluorescence.

SEM imaging. Molecular layers on HOPG or $\mathrm{MoS}_{2}$ were imaged using a Teneo VS SEM (FEI Company, Hillsboro, OR). Images were acquired at a working distance of $\sim 5 \mathrm{~mm}$ using the segmented in-lens T3 detector. A beam current of $3.2 \mathrm{nA}$ was selected for optimal image resolution, utilizing a 32- $\mu \mathrm{m}$ diameter aperture with an accelerating voltage of $5 \mathrm{kV}$. All substrates were affixed to standard SEM pin stub specimen mounts with double-sided conductive carbon tape and colloidal silver paint.

AFM imaging. All AFM measurements were performed under ambient conditions using a Bruker MultiMode AFM (Bruker Instruments, Billerica, MA) equipped with an E scanner. The setpoint ratio was typically maintained between 0.4 and 0.7 and was rarely decreased below 0.4 to avoid tip sweeping effects.

\section{AUTHOR INFORMATION}

\section{Corresponding Author}

*Address correspondence to: claridge@purdue.edu

Notes

The authors declare no competing financial interests.

\section{ACKNOWLEDGMENT}

SAC acknowledges support through a DARPA Young Faculty Award, N66001-17-1-4046. TCD acknowledges support through a Cagiantas graduate fellowship.

\section{REFERENCES}

1 Eigler, D. M. \& Schweizer, E. K. Positioning single atoms with a scanning tunneling microscope. Nature 344, 56-58 (1990).

2 Stroscio, J. A. \& Eigler, D. M. Atomic and molecular manipulation with the scanning tunneling microscope. Science 254, 1319-1326 (1991).

3 Cai, J. et al. Atomically precise bottom-up fabrication of graphene nanoribbons. Nature 466, 470-473 (2010).

4 Zwaneveld, N. A. A. et al. Organized formation of 2D extended covalent organic frameworks at surfaces. J. Am. Chem. Soc. 130, 66786679 (2008)

5 Sakaguchi, H., Matsumura, H., Gong, H. \& Abouelwafa, A. M. Direct visualization of the formation of single-molecule conjugated polymers. Science 310, 1002-1006 (2005).

6 Okawa, Y. \& Aono, M. Materials science - nanoscale control of chain polymerization. Nature 409, 683-684 (2001).

7 Hla, S. W., Bartels, L., Meyer, G. \& Rieder, K. H. Inducing all steps of a chemical reaction with the scanning tunneling microscope tip: Towards single molecule engineering. Phys. Rev. Lett. 85, 2777-2780 (2000).

8 Love, J. C., Estroff, L. A., Kriebel, J. K., Nuzzo, R. G. \& Whitesides, G. M. Self-assembled monolayers of thiolates on metals as a form of nanotechnology. Chem. Rev. 105, 1103-1169 (2005).

9 Rabe, J. P. \& Buchholz, S. Commensurability and mobility in 2-dimensional molecular patterns on graphite. Science 253, 424-427 (1991).

10 Groszek, A. J. Preferential adsorption of normal hydrocarbons on cast iron. Nature 196, 531-533 (1962).

11 Groszek, A. J. Preferential adsorption of long-chain normal paraffins on $\mathrm{MoS}_{2}$, WS 2 and graphite from n-heptane. Nature 204, 680 (1964).

Grill, L. \& Hecht, S. Covalent on-surface polymerization. Nat. Chem. 12, 115-130 (2020).

Campbell, A. J. \& Davies, C. K. L. Chain-length effects in polydiacetylene yellow solutions. Polymer 35 (1994).

Rawiso, M. et al. Solutions of polydiacetylenes in good and poor solvents: A light and neutron scattering study. J. Phys. 49, 861-880 (1988).

Okawa, Y., Akai-Kasaya, M., Kuwahara, Y., Mandal, S. K. \& Aono, M. Controlled chain polymerisation and chemical soldering for single-molecule electronics. Nanoscale 4, 3013-3028 (2012).

16 Hayes, T. R. et al. Multimicrometer noncovalent monolayer domains on layered materials through thermally controlled LangmuirSchaefer conversion for noncovalent 2D functionalization. ACS Appl. Mater. Interf. 9, 36409-36416 (2017).

17 Davis, T. C., Bang, J. J., Brooks, J. T., McMillan, D. G. \& Claridge, S. A. Hierarchically patterned noncovalent functionalization of 2D materials by controlled Langmuir-Schaefer conversion. Langmuir 34, 1353-1362 (2018).

18 Davis, T. C., Bechtold, J. O., Hayes, T. R., Villarreal, T. A. \& Claridge, S. A. Hierarchically patterned striped phases of phospholipids: Toward controlled presentation of carbohydrates. Faraday Discuss. 219, 229-243 (2019).

Tieke, B., Wegner, G., Naegele, D. \& Ringsdorf, H. Polymerization of tricosa-10,12-diynoic acid in multilayers. Angew. Chem., Int. Ed. Engl. 15, 764-765 (1976).

20

Wegner, G. Topochemical reactions of monomers with conjugated triple bonds .I. Polymerization of 2.4-hexadiyn-1.6-diols derivatives in crystalline state. Z. Naturforsch., B: J. Chem. Sci. B 24, 824-826 (1969). 
Lieser, G., Tieke, B. \& Wegner, G. Structure, phase-transitions and polymerizability of multilayers of some diacetylene monocarboxylic acids. Thin Solid Films 68, 77-90 (1980).

Reppy, M. A. \& Pindzola, B. A. Biosensing with polydiacetylene materials: Structures, optical properties and applications. Chem. Commun. 42, 4317-4338 (2007).

Porter, A. G. et al. 1-nm-wide hydrated dipole arrays regulate AuNW assembly on striped monolayers in nonpolar solvent. Chem 5, 2264-2275 (2019).

24 Heikenfeld, J. et al. Wearable sensors: Modalities, challenges, and prospects. Lab Chip 18, 217-248 (2018).

Zhou, J., Ellis, A. V. \& Voelcker, N. H. Recent developments in PDMS surface modification for microfluidic devices. Electrophoresis 31, 2-16 (2010).

Regehr, K. J. et al. Biological implications of polydimethylsiloxane-based microfluidic cell culture. Lab Chip 9, 2132-2139 (2009). Whitesides, G. M. Soft robotics. Angew. Chem., Int. Ed. 57, 4258-4273 (2018).

Wolf, M. P., Salieb-Beugelaar, G. B. \& Hunziker, P. PDMS with designer functionalities - properties, modificaion strategies, and applications. Prog. Polym. Sci. 83, 97-134 (2018).

Choong, S. W., Russell, S. R., Bang, J. J., Patterson, J. K. \& Claridge, S. A. Sitting phase monolayers of polymerizable phos pholipids create dimensional, molecular-scale wetting control for scalable solution based patterning of layered materials. ACS Appl. Mater. Interf. 9, 19326-19334 (2017).

Miura, A. et al. Light- and STM-tip-induced formation of one-dimensional and two-dimensional organic nanostructures. Langmuir 19, 6474-6482 (2003).

Giridharagopal, R. \& $1580(2008)$ Okawa, Y. \& Aono, M. Linear chain polymerization initiated by a scanning tunneling microscope tip at designated positions. J. Chem. Phys. 115, 2317-2322 (2001). Davis, T. C., Bang, J. J., Brooks, J. T., McMillan, D. G. \& Claridge, S. A. Hierarchical noncovalent functionalization of 2D materials by controlled Langmuir-Schaefer conversion. Langmuir 34, 1353-1362 (2018).

Bang, J. J., Porter, A. G., Davis, T. C., Hayes, T. R. \& Claridge, S. A. Spatially controlled noncovalent functionalization of 2D materials based on molecular architecture Langmuir 34, 5454-5463 (2018). or bis-silylated adducts. J. Org. Chem. 84, 2358-2365 (2019).

Tieke, B., Lieser, G. \& Wegner, G. Polymerization of diacetylenes in multilayers. J. Polym. Sci. A 17, 1631-1644 (1979).

Bubeck, C., Tieke, B. \& Wegner, G. Selfsensitization of the photopolymerization of diacetylenes studied in multilayers. Ber. BunsenGes. 86, 495-498 (1982).

Olmsted, J. \& Strand, M. Fluorescence of polymerized diacetylene bilayer films. J. Phys. Chem. 87, 4790-4792 (1983).

Geiger, E., Hug, P. \& Keller, B. A. Chromatic transitions in polydiacetylene Langmuir-Blodgett films due to molecular recognition at the film surface studied by spectroscopic methods and surface analysis. Macromol. Chem. Phys. 203, 2422-2431 (2002).

Bloor, D. \& Preston, F. H. Optical properties of bis(p-toluene sulphonate) diacetylene. I. Crystal optics of partially and fully polymerized crystals. Phys. Status Solidi A 37, 427-438 (1976).

Bloor, D. \& Preston, F. H. Optical properties of bis(p-toluene sulphonate) diacetylene. II. Low-temperature spectra. Phys. Status Solidi A 39, 607-614 (1977).

Combescot, M. \& Guillet, T. Excitons in quantum wires. Eur. Phys. J. B 34, 9-24 (2003).

Schott, M. The colors of polydiacetylenes: A commentary. J. Phys. Chem. B 110, 15864-15868 (2006).

Lécuiller, R., Berréhar, J., Lapersonne-Meyer, C. \& Schott, M. Dual resonance fluorescence of polydiacetylene chains isolated in their crystalline monomer matrix. Phys. Rev. Lett. 80, 4068-4071 (1998).

Davis, T. C., Russell, S. R. \& Claridge, S. A. Edge-on adsorption of multi-chain functional alkanes stabilizes noncovalent monolayers on MoS. Chem. Commun. 54, 11709-11712 (2018).

Takami, K. et al. Significant increase in conductivity of polydiacetylene thin film induced by iodine doping. Surf. Sci. 591, L273L279 (2005).

Yuan, C. W., Lu, C. L., Liang, Y., Gu, N. \& Wei, Y. Colour transition of polydiacetylene multilayers caused by their interaction with several kinds of gases. Solid State Commun. 80, 493-495 (1991).

Gaboriaud, F., Volinsky, R., Berman, A. \& Jelinek, R. Temperature dependence of the organization and molecular interactions within phospholipid/diacetylene Langmuir films. J. Colloid Interface Sci. 287, 191-197 (2005).

Lin, M. H., Chen, C. F., Shiu, H. W., Chen, C. H. \& Gwo, S. Multilength-scale chemical patterning of self-assembled monolayers by spatially controlled plasma exposure: Nanometer to centimeter range. J. Am. Chem. Soc. 131, 10984-10991 (2009). 


\title{
Supporting Information
}

\section{1-nm Resolution Functional Pattern Transfer to an Amorphous Elastomeric Material}

\author{
Tyson C. Davis,${ }^{\dagger}$ Jeremiah O. Bechtold,${ }^{\dagger}$ Anni Shi, ${ }^{\dagger}$ Erin N. Lang,${ }^{\dagger}$ Anamika Singh, ${ }^{\dagger}$ \\ Shelley A. Claridge,,,$+ \neq$ \\ †Department of Chemistry, Purdue University, West Lafayette, IN 47907 \\ ¥Weldon School of Biomedical Engineering, Purdue University, West Lafayette, IN 47907 \\ *Address correspondence to: claridge@purdue.edu, (phone) 765-494-6070
}

\section{MATERIALS AND METHODS}

Materials. Chloroform ( $\geq 99.5 \%$ purity), platinum(IV) oxide (surface area $\geq 75 \mathrm{~m}^{2} / \mathrm{g}$ ), xylenes ( $\geq 98.5 \%$ ), iodine (99.8 \%) triisopropylsilane (TIPS) (98\% purity), oleylamine (technical grade, $70 \%$ purity), and molecular sieves (4 $\AA$ ) were purchased from MilliporeSigma (St. Louis, MO) and used as received. Absolute ethanol (100\% purity) was purchased from Decon Laboratories, Inc. (King of Prussia, PA) and used as received. Methanol ( $\geq 99.8 \%)$, hexanes ( $\geq 98.5 \%$ ), dimethyl sulfoxide (DMSO, $\geq 99.9 \%$ purity), isopropanol (IPA, $\geq 99.5 \%$ purity), calcium chloride, cadmium chloride, and Rhodamine Red-X succinimidyl ester were all purchased from Fisher Scientific (Hampton, NH) and used as received. Triethylsilane (98.0\% purity) was purchased from Gelest (Morrisville, PA) and used as received. 1,2-Bis(10,12-tricosadiynoyl)-sn-glycero-3phosphoethanolamine (dPE, >99.0 \% purity) was purchased from Avanti Polar Lipids (Alabaster, AL) and used as received. 10,12-Pentacosadiynoic acid (PCDA, $\geq 97.0 \%$ purity) and 10,12-tricosadiynoic acid (TCDA, $\geq 98.0 \%$ purity) were purchased from Sigma-Aldrich (St. Louis, MO), dissolved in chloroform, and filtered using a 13-mm syringe filter with a PTFE membrane and $0.2-\mu \mathrm{m}$ pores (VWR, Radnor, PA). For transfer to polydimethylsiloxane (PDMS), SYLGARD 184 silicone elastomer kits containing base and curing (crosslinking) agent were purchased from Dow Chemical Company (Midland, MI). Cyclohexane (99.5\% purity, extra dry) and tetrachloroauric(III) acid trihydrate (ACS reagent grade, $\geq 49.0 \%$ Au purity) were purchased from ACROS Organics (Fair Lawn, NJ) and used as received. Milli-Q water ( $\geq 18.2 \mathrm{M} \Omega \cdot \mathrm{cm}$ resistivity) was used whenever water was required in an experiment. Ultrahigh purity nitrogen (UHP $\mathrm{N}_{2}, 99.999 \%$ purity) was purchased from Indiana Oxygen Company (Indianapolis, IN). Lipids were deposited on either $1 \mathrm{~cm} \times 1 \mathrm{~cm}$ highly oriented pyrolytic graphite (HOPG, MicroMasch, Watsonville, CA) substrates or $\sim 1 \mathrm{~cm}^{2}$ molybdenum disulfide (MoS ${ }_{2}$, SPI Supplies, West Chester, PA) substrates; substrates were cleaved immediately prior to sample deposition. All initial steps in the transfer process were carried out under UV-filtered light to prevent unwanted polymerization. PELCO conductive liquid silver paint, standard SEM pin stub mounts, AFM specimen discs (alloy 430), PELCO Formvar/carbon 400 mesh TEM grid, and double-coated carbon conductive tape were purchased from Ted Pella, Inc. (Redding, CA).

Preparation of PCDA monolayers on HOPG. Unless otherwise stated in the manuscript, PCDA monolayers were prepared using a previously reported ${ }^{1}$ Langmuir-Schaefer (LS) conversion method, under conditions that induce microscopic vacancies in the film. The microscopic vacancies provide enhanced fluorescence contrast after transfer to PDMS, and allowing individual domains within the vacancies to be resolved. LS conversion was performed using a MicroTrough XL Langmuir-Blodgett trough (Kibron Inc., Helsinki, Finland). A PCDA film was assembled at the air-water interface by depositing $30 \mu \mathrm{L}$ of a $0.5 \mathrm{mg} / \mathrm{mL}$ solution of PCDA in chloroform on a subphase of Milli-Q water $(\sim 18 \mathrm{M} \Omega \cdot \mathrm{cm})$ at $30^{\circ} \mathrm{C}$. The chloroform was allowed to evaporate for $15 \mathrm{~min}$ before the trough barriers were slowly moved inward $(8.5 \mathrm{~mm} / \mathrm{min}$ barrier motion) until the target mean molecular area of $30 \AA^{2} /$ molecule was reached. A freshly cleaved HOPG substrate was mounted on an automated dipper attachment, with the HOPG oriented nearly parallel to the air-water interface (cleaved side facing down), then lowered horizontally at a rate of $6 \mathrm{~mm} / \mathrm{min}$. After $2 \mathrm{~min}$ of contact with the interface, the HOPG was gently lifted out of contact with the liquid at the 
same speed. After contact with the interface was broken, the substrate was blown dry with UHP $\mathrm{N}_{2}$. Finally, unless otherwise stated, the substrate was placed under a hand-held UV lamp $(254 \mathrm{~nm}, 8 \mathrm{~W})$ for $1 \mathrm{~h}$ with $\sim 2 \mathrm{~cm}$ between the lamp and the substrate, to induce diyne photopolymerization, stabilizing the assembled molecular layer.

Preparation of PDMS stamps for microcontact printing ( $\mu \mathrm{CP})$. PDMS stamps were prepared using a modification of a previously published lithography-free method. ${ }^{2}$ Formvar-and-carbon-coated copper 400 mesh TEM grids (approximate grid hole size $42 \mu \mathrm{m}$ ) were placed Formvar side down in a glass Petri dish. Stamps were prepared by mixing SYLGARD 184 silicone elastomer base and curing (crosslinking) agent at a $10: 1(\mathrm{~m} / \mathrm{m}$ ) ratio. After the components were thoroughly mixed (ca. $5 \mathrm{~min}$ ), the mixture was poured onto the TEM grids. The mixture was then deaerated in a vacuum desiccator until no bubbles remained. The PDMS was cured for $2 \mathrm{~h}$ at $80^{\circ} \mathrm{C}$; the TEM grids were then gently peeled from the PDMS, generating a micropatterned surface. The resulting stamps were cleaned by sonication in a 1:1:1 $(\mathrm{v} / \mathrm{V} / \mathrm{V})$ mixture of ethanol, methanol, and Milli-Q water for $60 \mathrm{~min}$. Following sonication, stamps were placed in an oven for $1 \mathrm{~h}$ at $60^{\circ} \mathrm{C}$ to allow residual polar solvent mixture to evaporate. Stamps were then soaked in hexanes for $6 \mathrm{~h}$, replacing the hexanes every $2 \mathrm{~h}$. Finally, the stamps were dried for $24 \mathrm{~h}$ at $60^{\circ} \mathrm{C}$ and placed, pattern side up, in a covered Petri dish prior to use.

$\mu \mathrm{CP}$ of PCDA or other striped monolayers. Inking and printing steps were performed following a previously published procedure, ${ }^{3}$ described briefly here. The molecular ink solution was prepared by diluting a $2.5 \mathrm{mg} / \mathrm{mL}$ solution of PCDA in $3: 2$ hexanes:IPA (v/v) with ethanol to a final PCDA concentration of $0.4 \mathrm{mg} / \mathrm{mL}$. The PDMS stamp was briefly rinsed with ethanol and blown dry with UHP $\mathrm{N}_{2}$ prior to immersing the stamp in the ink solution for $30 \mathrm{~s}$. The stamp was then removed, blown dry with UHP $\mathrm{N}_{2}$, and placed pattern side up at room temperature for $1 \mathrm{~h}$. Subsequently, the patterned (inked) side of the PDMS stamp was brought into contact with a freshly cleaved HOPG substrate for $30 \mathrm{~s}$ and then carefully lifted from the surface. Finally, the printed HOPG substrate was placed under a hand-held UV lamp (254 nm, $8 \mathrm{~W})$ for $1 \mathrm{~h}$ with $\sim 2 \mathrm{~cm}$ between the lamp and the substrate to induce diyne photopolymerization. A similar process was followed for $\mu \mathrm{CP}$ of other amphiphiles used in the manuscript.

Preparation of dPE monolayers on HOPG and $\mathrm{MoS}_{2}$. Unless otherwise stated in the manuscript, dPE films were prepared using temperature-controlled LS conversion to achieve complete coverage. The temperature-controlled LS conversion process utilizes a temperature-controlled transfer stage that was reported previously, ${ }^{4}$ in conjunction with a MicroTrough $\mathrm{XL}$ Langmuir-Blodgett trough. Samples were mounted on the stage with standard 12-mm diameter stainless-steel AFM specimen discs that mount on a magnet recessed in the body of the stage. To maximize temperature uniformity across the substrate surface, conductive carbon tape was used to affix the back of the substrate to the specimen disc surface. The temperature of the substrate was measured using a thermocouple prior to dipping.

The dPE film was created at the air-water interface by depositing $30 \mu \mathrm{L}$ of $0.50 \mathrm{mg} / \mathrm{mL} \mathrm{dPE}$ in chloroform on a subphase of Milli-Q water at $30{ }^{\circ} \mathrm{C}$. The chloroform was allowed $15 \mathrm{~min}$ to evaporate before the trough barriers were slowly moved inward $\left(8.5 \mathrm{~mm} / \mathrm{min}\right.$ barrier motion) until the target surface pressure of $30 \mathrm{mN} / \mathrm{m}$ was reached. Freshly cleaved $\mathrm{HOPG}_{\text {or }}$ MoS $_{2}$ was heated to $50{ }^{\circ} \mathrm{C}$ using the thermally controlled dipping attachment, then lowered into contact with the subphase at $6 \mathrm{~mm} / \mathrm{min}$. The substrate was left in contact with the interface for $2 \mathrm{~min}$ and then withdrawn from the interface using the automated dipper at the same speed. After contact with the interface was broken, the substrate was blown dry with UHP $\mathrm{N}_{2}$. Finally, unless otherwise stated, the substrate was placed under a hand-held UV lamp ( $254 \mathrm{~nm}, 8 \mathrm{~W}$ ) for $1 \mathrm{~h}$ with $\sim 2 \mathrm{~cm}$ between the lamp and the substrate, to induce diyne photopolymerization, stabilizing the assembled molecular layer.

Preparation of the PCDA-dPE mixed monolayer film. Mixed monolayers of PCDA and dPE on HOPG were prepared with a temperature-controlled transfer and a MicroTrough XL Langmuir-Blodgett trough. A 1:1 mixture of PCDA:dPE was prepared by mixing equal volumes of a $0.5 \mathrm{mg} / \mathrm{mL}$ solution of PCDA in chloroform with a $0.5 \mathrm{mg} / \mathrm{mL}$ solution of dPE in chloroform to yield a solution with a concentration of $0.25 \mathrm{mg} / \mathrm{mL}$ of each amphiphile in chloroform. The PCDA-dPE film was then assembled at the air-water interface by spreading $18 \mu \mathrm{L}$ of the solution on a subphase of Milli-Q water at $30{ }^{\circ} \mathrm{C}$. The chloroform was allowed to evaporate for $60 \mathrm{~min}$ before the trough barriers were slowly moved inward $(6.23 \mathrm{~mm} / \mathrm{min}$ barrier motion) until the target surface pressure of $8 \mathrm{mN} / \mathrm{m}$ was reached. A freshly cleaved HOPG substrate was mounted on an automated dipper attachment, with the HOPG oriented nearly parallel to the air-water interface (cleaved side facing down), then lowered horizontally at a rate of $6 \mathrm{~mm} / \mathrm{min}$. After $4 \mathrm{~min}$ of contact with the interface, the HOPG was gently lifted out of contact with the liquid at the same speed. After contact with the interface was broken, the substrate was blown dry with UHP $\mathrm{N}_{2}$. Finally, the substrate was placed under a hand-held UV lamp $(254 \mathrm{~nm}, 8 \mathrm{~W})$ for $1 \mathrm{~h}$ with $2 \mathrm{~cm}$ between the lamp and the substrate, to induce diyne photopolymerization.

Hydrosilylation reactions of dPE and TCDA with triethylsilane. To establish whether single- and dual-chain diacetylenes were susceptible to hydrosilylation, reactions were performed using a modification of published procedures. ${ }^{5-8}$ Reactions were carried out under an $\mathrm{N}_{2}$ atmosphere using standard Schlenk line techniques. A fresh stir bar and fresh glassware were used in all reactions to avoid contamination. $\mathrm{PtO}_{2}(0.05 \mathrm{mmol})$ and the amphiphile $(1 \mathrm{mmol} \mathrm{dPE}$ or TCDA) were added to a $20-\mathrm{mL}$ 
round-bottom flask. The flask was then purged with $\mathrm{N}_{2}$ for $2 \mathrm{~h}$; subsequently, enough xylene $(\sim 2 \mathrm{~mL})$ was added via syringe to dissolve the amphiphile. Triethylsilane was then introduced via syringe. The flask was then placed in an oil bath at $60{ }^{\circ} \mathrm{C}$ and the reaction mixture was stirred for $1 \mathrm{~h}$. $\mathrm{PtO}_{2}$ was filtered out of the reaction mixture using a 13-mm syringe filter with a PTFE membrane and $0.2-\mu \mathrm{m}$ pores. The filtrate was further diluted with xylenes $(\sim 10 \mathrm{~mL})$ prior to removal of an aliquot for analysis of reaction products by mass spectrometry.

Covalent transfer of polymerized diyne amphiphile striped phases from HOPG to PDMS. Unless otherwise stated, transfer of polymerized diyne amphiphile striped phases was performed using the following procedure. SYLGARD 184 silicone elastomer base and curing (crosslinking) agent were mixed at a 10:1 (m/m) ratio. After the components were thoroughly mixed (ca. $5 \mathrm{~min}$ ), the mixture was poured onto the HOPG substrate functionalized with the polymerized diyne amphiphile film. The mixture was then deaerated in a vacuum desiccator until no bubbles remained. The PDMS was cured for $24 \mathrm{~h}$ at $60{ }^{\circ} \mathrm{C}$, also allowing the PDMS to react with the polymerized monolayer. The cured (solid) PDMS was then peeled away from the HOPG substrate, resulting in transfer of the polymerized diyne amphiphile film to the free PDMS.

lodine exposure to PCDA films. PCDA films on HOPG were placed in a sealed chamber saturated with $\mathrm{I}_{2}$ vapor for 5 min. The substrates were then placed under vacuum for $24 \mathrm{~h}$ to sublime any physisorbed iodine. Finally, $\mathrm{I}_{2}$-exposed PCDA films were transferred to PDMS using the procedure described above.

AuNW synthesis and adsorption onto PDMS. AuNWs were prepared by modifying a previously reported synthetic protocol. ${ }^{9-}$ 12 Briefly, to a vial containing molecular sieves, $2.5 \mathrm{mg}$ of $\mathrm{HAuCl}_{4} \cdot 3 \mathrm{H}_{2} \mathrm{O}$ and $2.2 \mathrm{~mL}$ of cyclohexane were added. Next, oleylamine $(84 \mu \mathrm{L})$ was added and the reaction was agitated using a vortex mixer. Triisopropylsilane $(120 \mu \mathrm{L})$ was added, and the mixture was immediately vortexed until the $\mathrm{HAuCl}_{4} \cdot 3 \mathrm{H}_{2} \mathrm{O}$ was dissolved. The reaction was moved into a humidity-controlled environment and aged for $12 \mathrm{~h}$. After aging, an aliquot of AuNW growth solution was diluted by a factor of 10 with dry cyclohexane. A dPE/PDMS substrate was lowered into contact with the dilute AuNW solution for 2 min, rinsed with $1 \mathrm{~mL}$ of dry cyclohexane, and dried under a stream of $\mathrm{N}_{2}$.

Synthesis of 10,12-pentacosadiynamine (PCD- $\mathrm{NH}_{2}$ ). Synthesis was carried out using a modification of previously reported literature procedures, described briefly here. ${ }^{13-15}$ First, 10,12-Pentacosadynoic acid (PCDA) (1 eq, 500 mg) was dissolved in anhydrous DCM (10 mL) under $\mathrm{N}_{2}$ atmosphere. Oxalyl chloride $(1.3 \mathrm{eq}, 0.17 \mathrm{~mL}$ ) and DMF (N, N-dimethylformamide) (2 drops) were added to the solution. The reaction mixture was stirred at room temperature overnight and concentrated under reduced pressure to obtain 10,12-pentacosadiynoyl chloride (PCD-Cl) as yellow oil which was used for the next step without further purification. Next, in a round-bottom flask, 28-30\% aqueous ammonium hydroxide (1.3 eq, $32 \mathrm{~mL}$ ) was added. PCD$\mathrm{Cl}(1 \mathrm{eq}, 500 \mathrm{mg}$ ) was dissolved in THF $(10 \mathrm{~mL})$ and the resulting solution was added to the ammonium hydroxide solution at $0{ }^{\circ} \mathrm{C}$. The reaction mixture was stirred at room temperature for $6 \mathrm{~h}$. The product was extracted with $\mathrm{DCM}(3 \times 50 \mathrm{~mL})$ and the combined organic extract was dried over anhydrous $\mathrm{Na}_{2} \mathrm{SO}_{4}$. The DCM was evaporated under reduced pressure to yield 10,12-pentacosadiynoyl amide (308 mg, 65\%) as white solid. 10,12-Pentacosadiynoyl amide (1 eq, $200 \mathrm{mg}$ ) was placed in a round-bottom flask. Anhydrous diethyl ether $(20 \mathrm{~mL})$ was added to the flask under $\mathrm{N}_{2}$ atmosphere, yielding a white suspension. Subsequently, $\mathrm{LiAlH}_{4}(10 \mathrm{eq}, 203 \mathrm{mg})$ was added to the suspension at $0{ }^{\circ} \mathrm{C}$ and the reaction mixture was stirred at room temperature for $20 \mathrm{~h}$. After the reaction was complete, the mixture was cooled to $0{ }^{\circ} \mathrm{C}$ and treated with sequential dropwise addition of water $(0.2 \mathrm{~mL})$, aqueous $\mathrm{NaOH}(15 \% \mathrm{w} / \mathrm{w}, 0.2 \mathrm{~mL})$ and water $(0.6 \mathrm{~mL})$. The mixture was filtered to remove inorganic impurities. Finally, the filtrate was dried over anhydrous $\mathrm{Na}_{2} \mathrm{SO}_{4}$ to yield $\mathrm{PCD}-\mathrm{NH}_{2}(175 \mathrm{mg}, 91 \%)$ as white solid.

Reaction of dPE/PDMS or PCD-NH $\mathrm{NHDMS}_{2}$ with RR succinimidyl ester. The reaction to link RR to the primary amines of $\mathrm{dPE} / \mathrm{PDMS}$ or PCD-NH $\mathrm{N}_{2} / \mathrm{PDMS}$ substrates was performed by first dissolving $2.3 \mathrm{mg}$ of RR succinimidyl ester in $1.5 \mathrm{~mL}$ dimethylformamide (DMF). The solution was placed in a v-vial containing a dPE/PDMS or $\mathrm{PCD}-\mathrm{NH}_{2} / \mathrm{PDMS}$ substrate under a $\mathrm{N}_{2}$ environment. The vial was placed in a Falcon tube and slowly vortexed overnight. Substrates were then rigorously washed to minimize physisorbed RR retention on the PDMS surface or in pores; washing steps were based on protocols commonly employed to remove unreacted PDMS oligomers from PDMS stamps prepared for $\mu \mathrm{CP} .{ }^{16}$ Functionalized PDMS substrates were rinsed thoroughly with ethanol and then with Milli-Q water. Subsequently, the PDMS substrates were placed in a Falcon tube and sonicated in DMF for $2 \mathrm{~h}$, replacing the DMF every hour. Finally, PDMS substrates were sonicated in a mixture of 1:1:1:1:1 (v/v/v/v/v) methanol, ethanol, isopropanol, Milli-Q water, and DMSO and sonicated for $2 \mathrm{~h}$, then dried for $6 \mathrm{~h}$ in an oven at $60^{\circ} \mathrm{C}$ prior to imaging.

Contact angle titrations. Contact angle titrations were performed using an Attension Theta optical tensiometer (Biolin Scientific, Espoo, Finland). Buffers with $20 \mathrm{mM}$ buffering capacity at $\mathrm{pH}$ values from 2 to 13 were utilized. For each measurement, a 3- $\mu \mathrm{L}$ droplet of a buffer solution was applied to the functionalized PDMS substrate. The tensiometer recorded images at video rates as each droplet was applied to the surface (for the advancing angle measurement) and as liquid was slowly withdrawn from the droplet using a 32-gauge needle until the solvent front receded (for the receding angle measurement). Images were then analyzed using the OneAttension software package, in sessile droplet mode, based on the 
Young-Laplace equation. Each contact angle graphed in the manuscript represents the average of 9-27 data points (typically three points measured on each of at least three different samples). Typically, it was possible to acquire nine measurements in a grid across each $1 \mathrm{~cm}^{2}$ sample.

Wide-field fluorescence microscopy. Wide-field fluorescence micrographs were obtained using an Olympus BX-51 optical microscope with an Olympus DP71 color camera. Images were acquired using either a 40X (metallographic, plan-fluorite aberration correction, $\mathrm{NA}=0.75$, infinity corrected optics), or a 100X (metallographic, apochromatic and flat field correction, $\mathrm{NA}=0.95$ ) brightfield objective. A UMWB2 filter cube was utilized with an 460-490 nm excitation band-pass filter, a dichroic filter wavelength of $500 \mathrm{~nm}$ and a long-pass emission filter wavelength of $520 \mathrm{~nm}$. Typical image collection times were $60 \mathrm{~s}$ with a resolution of $1024 \times 1024$ pixels. For polarized emission images, an Olympus U-AN360P 360-degree rotatable analyzer with a minimum angle of 0.1 degree was placed in the emission path between the filter cube and the camera.

Confocal fluorescence microscopy and spectral imaging. Confocal micrographs and associated spectra were acquired utilizing a Zeiss LSM 880, Axio Examiner upright confocal microscope. Unless otherwise stated in the manuscript, excitation was provided by a $488 \mathrm{~nm}$ Ar laser set to $100 \%$ power. Data were obtained using a 20X objective (plan-apochromatic, dry, $\mathrm{NA}=0.80$ ), with a $0.17-\mathrm{mm}$ cover glass placed on the sample, and a 32-channel GaAsP spectral photomultiplier tube detector. Micrographs and corresponding spectra were obtained at a resolution of 2856 pixels per line, a bit depth of 16 bits, a dwell time of $11.75 \mu \mathrm{s} / \mathrm{pixel}$ (using unidirectional scanning and averaging 16 times per line), and a pinhole set to 1 Airy unit. For detection, 23 of the 32 channels were used to collect data, with bins centered at values from 495-691 nm and a resolution (bin width) of $8.9 \mathrm{~nm}$. Bare PDMS spectra were subtracted from monolayer spectra during processing to minimize contributions from PDMS autofluorescence.

Exposure of PCDA/PDMS and dPE/PDMS monolayers to divalent cations. To determine the effect of divalent cations on monolayer fluorescence, as-prepared PCDA/PDMS and dPE/PDMS monolayers were first imaged, then a 150- $\mu \mathrm{L}$ droplet of the divalent ion solution (100 $\mathrm{mM} \mathrm{CaCl}_{2}$ or $\mathrm{CdCl}_{2}$ in Milli-Q water) was placed on the surface. Imaging was carried out as described above, with the exception that for immersive imaging, a 20X objective (W-plan-apochromatic, water dipping, $N A=1.00$ ) was dipped into the droplet; no cover slip was used for these measurements.

SEM imaging. Molecular layers on HOPG or $\mathrm{MoS}_{2}$ were imaged using a Teneo VS SEM (FEI Company, Hillsboro, OR). Images were acquired at a working distance of $\sim 5 \mathrm{~mm}$ using the segmented in-lens T3 detector. A beam current of $3.2 \mathrm{nA}$ was selected for optimal image resolution, utilizing a $32-\mu \mathrm{m}$ diameter aperture with an accelerating voltage of $5 \mathrm{kV}$. All substrates were affixed to standard SEM pin stub specimen mounts with double-sided conductive carbon tape. To further enhance substrate-mount conductivity, a small amount of colloidal silver paint was applied along the perimeter of the substrate, providing electrical contact with the underlying pin stub.

AFM imaging. All AFM measurements were performed under ambient conditions using a Bruker MultiMode AFM (Bruker Instruments, Billerica, MA) equipped with an E scanner. The cantilever oscillation phase shift was carefully monitored to ensure the tip was engaged in the attractive mode to improve imaging of lamellar domains. The setpoint ratio was typically maintained between 0.4 and 0.7 and was rarely decreased below 0.4 to avoid tip sweeping effects.

Image analysis. AFM and SEM micrographs were processed using Gwyddion scanning probe microscopy data visualization and analysis software. ${ }^{17}$ Confocal fluorescence micrographs and spectra were processed in ZEN (blue) software.

Energy minimization. Software packages Maestro and Macromodel (Schrödinger, Cambridge MA) were used, respectively, to visualize molecular structures and to perform force field minimizations. Models were minimized using the OPLS_2005 force field, with extended cutoffs for Van der Waals, electrostatic, and hydrogen bonding interactions. Explicit water was used in the simulations and the dielectric constant of the simulation was set to 80.1. Minimizations were performed using the Polak-Ribiere conjugate gradient (PRCG) algorithm and gradient method with 50000 runs and a convergence threshold of 0.05 . 


\section{Larger AFM and SEM images illustrating PCDA and dPE assembly on HOPG}

In the main manuscript, Fig. 2 shows AFM and SEM images illustrating lamellar structure and domain structure for PCDA and dPE on HOPG. Here, we show larger AFM and SEM images (Supplementary Figs. S1 and S2) to highlight the resolution with which it is possible to observe striped phase domain structure on HOPG via SEM and the length scale of ordering that is possible to achieve using this assembly strategy. Supplementary Fig. 1a shows an SEM image of PCDA striped phase assembled on HOPG (PCDA/HOPG). Throughout the image, long linear features generally oriented from upper right to lower left correspond to HOPG step edges. Small topographic asperities in the HOPG substrate (see lower right corner) are also in some cases visible.

Rows of PCDA assemble along three crystallographically equivalent $<11 \underline{20}>$ axes of the HOPG and are visible as more highly scattering areas of the micrograph. Darker areas in the micrograph correspond to vacancies in the monolayer. For some experiments in this manuscript, we utilized striped phase PCDA monolayers assembled on HOPG under conditions that result in rounded or oval microscopic vacancies in the monolayer, providing a means for comparing functionalized and unfunctionalized areas of the PDMS surface following transfer. Within microscopic vacancies (for example, in the upper right corner of Supplementary Fig. 1a), long, narrow molecular domains are often visible, which also provides a means for assessing fidelity of nanoscale domain transfer to PDMS. An AFM image showing the edge of a molecular domain (Supplementary Fig. 1b) contains an inset showing the measured topographic height difference at the domain edge (typically $0.3-0.4 \mathrm{~nm}$ ).

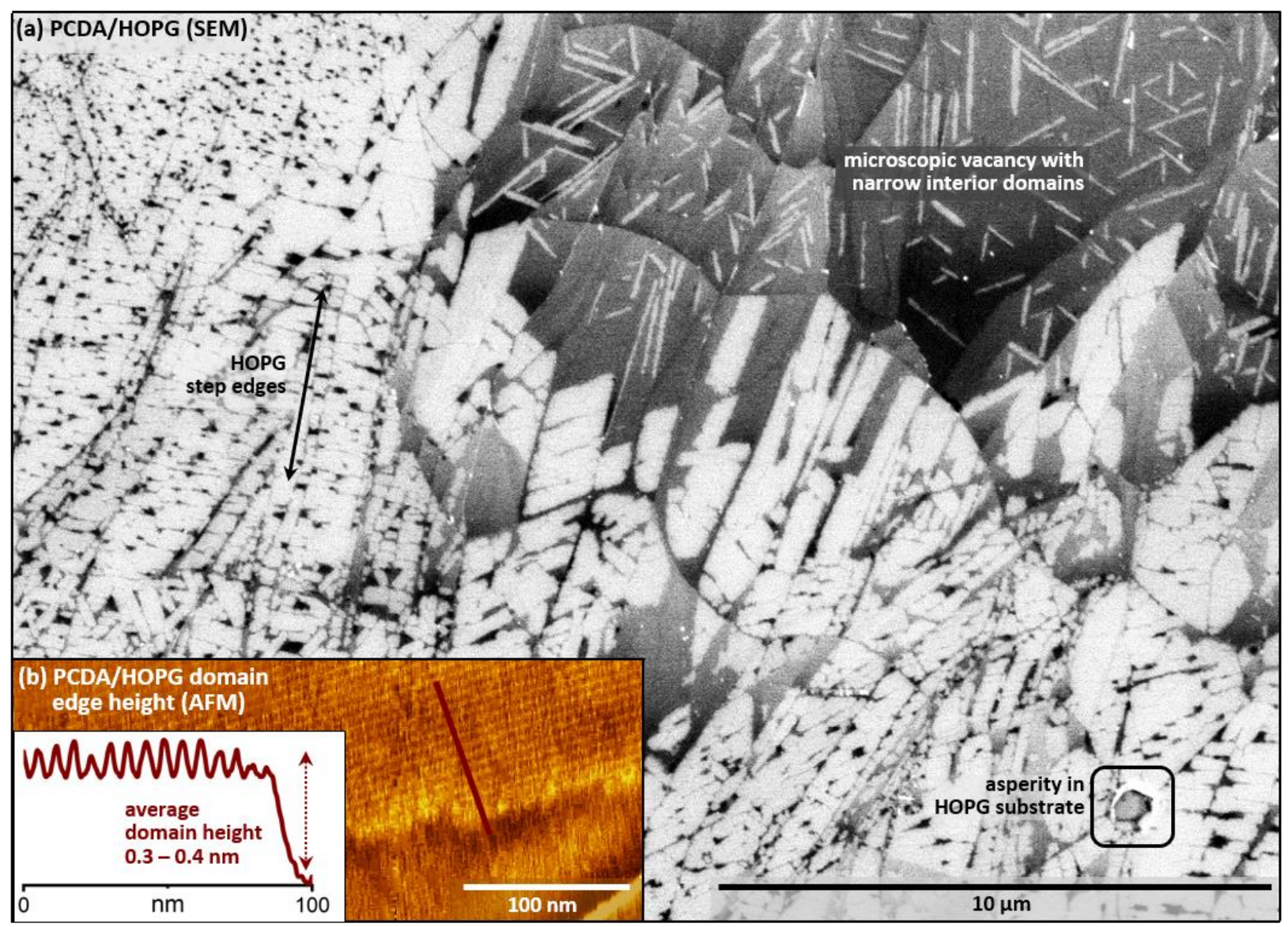

Figure 1. SEM image of PCDA/HOPG, highlighting commonly observed features arising from monolayer structure and from substrate structure. 
Supplementary Fig. 2a shows an SEM image of dPE assembled on HOPG (dPE/HOPG). Here, HOPG step edges run predominantly from upper left to lower right. Surface coverage is more complete than in the PCDA/HOPG monolayer in the previous figure, with molecular row orientation visible in large domains based on cracking defects that occur in the monolayer during polymerization. In some areas (such as the lower right corner of the image), small rounded standing phase domains are visible. Supplementary Fig. $2 b$ shows an AFM image of molecular ordering in a dPE striped phase domain, with the topographic height difference (typically $0.4-0.5 \mathrm{~nm}$ ) measured adjacent to a vacancy in the monolayer.

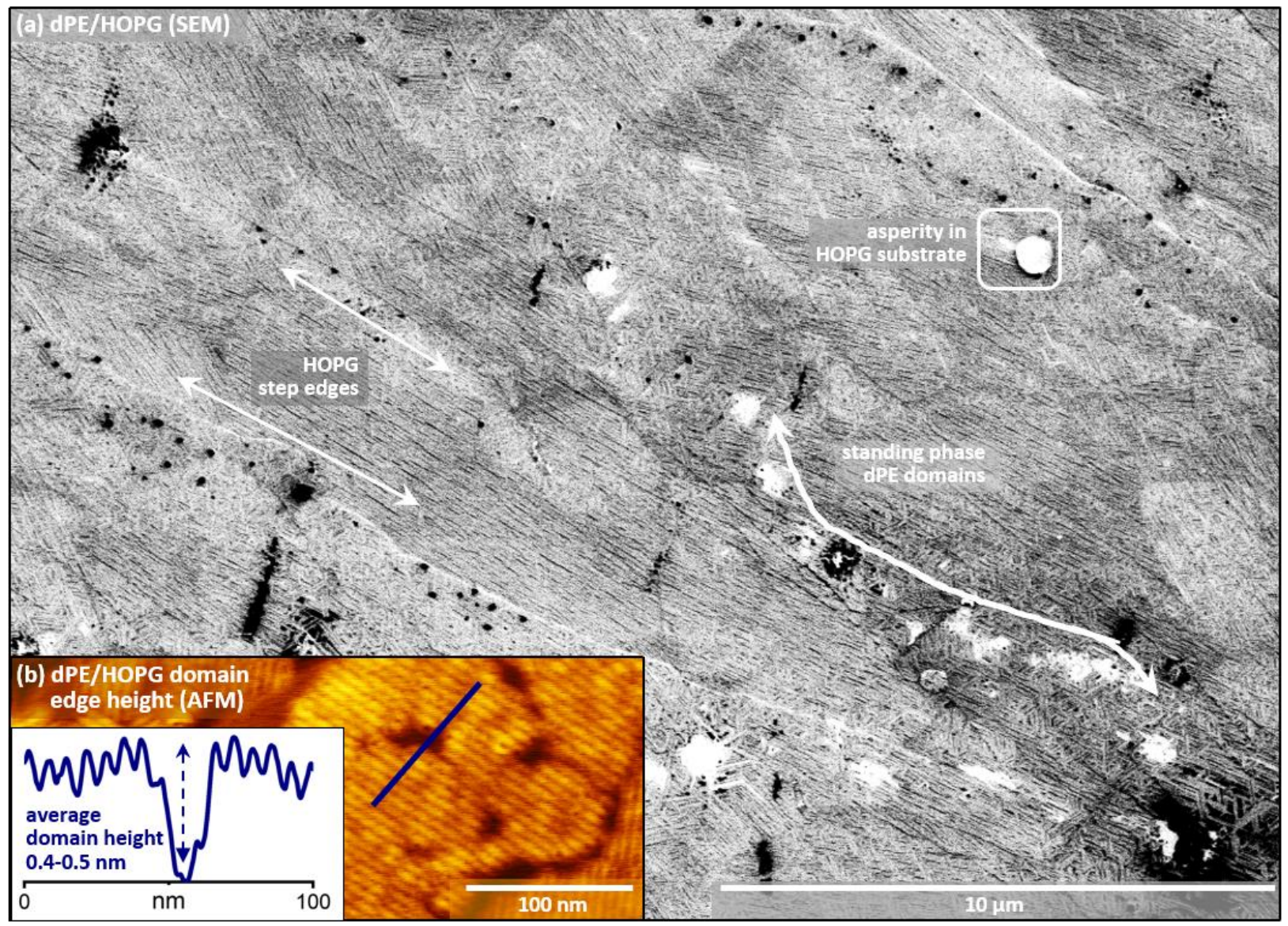

Figure 2. SEM image of dPE/HOPG, highlighting commonly observed features arising from monolayer structure and from substrate structure.

\section{Larger SEM images of PCDA/HOPG assembled through microcontact printing ( $\mu$ CP-PCDA/HOPG), and larger fluorescence image of $\mu \mathrm{CP}$-patterned striped phase patterns transferred to PDMS}

In the main manuscript, we utilize square patterns of PCDA and other striped phases on HOPG as substrates for a number of experiments. Here, we show two SEM images of PCDA striped phases on HOPG assembled by microcontact printing to provide more details of domain structure achieved using this assembly method. Supplementary Fig. 3a shows a pattern of $\sim 46-\mu \mathrm{m}$ squares of PCDA assembled on HOPG. Supplementary Fig. 3b shows an enlarged image of the edge of one square, in which narrow linear domains are visible, protruding from the edge of the square pattern. In both images, several HOPG step edges are visible, extending from upper right to lower left in the view area. Also visible are several small dark areas in the micrographs. Previous experiments have suggested that these are PDMS oligomers deposited during the microcontact printing process.

We note that in the initial deposition step (in which a solution of amphiphiles is deposited as an 'ink' from a square-patterned PDMS stamp onto the HOPG), to the best of our knowledge the amphiphiles do not form a striped phase directly on the PDMS stamp. Rather, subsequent application to the HOPG surfaces causes molecules to be converted into the ordered striped phases, generating the 1-nm-wide functional patterns that are then transferred to a new PDMS substrate generated by curing freshly mixed PDMS base and crosslinker on the striped phase on HOPG. 


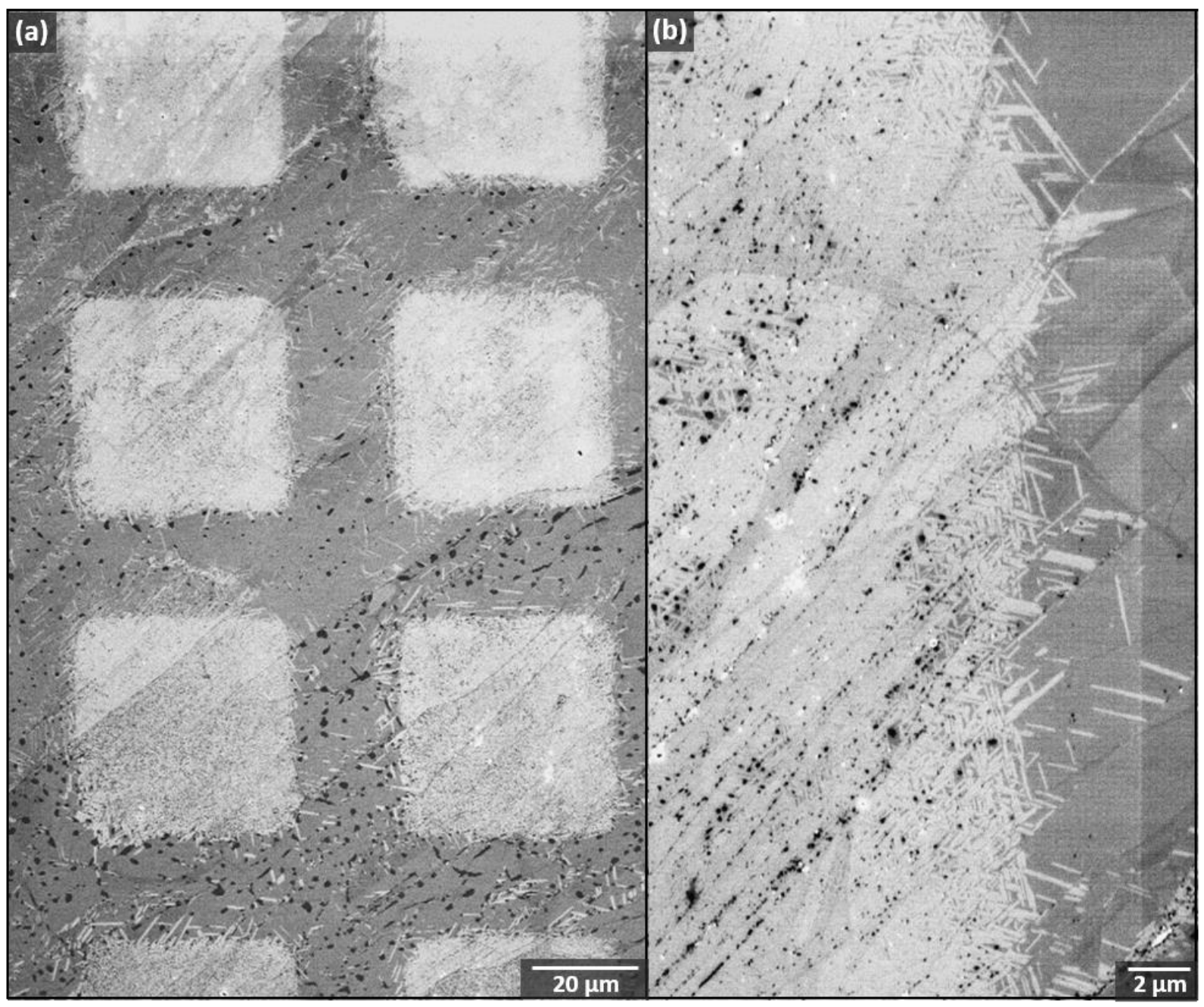

Figure 3. SEM images of (a) $\mu \mathrm{CP}-\mathrm{PCDA} / \mathrm{HOPG}$ and (b) the edge of one square, illustrating striped phase domain structure.

\section{Discussion of postulated transfer mechanism}

Several experiments support the hypothesis that a hydrosilylation reaction between $\mathrm{Si}-\mathrm{H}$ bonds in the PDMS crosslinker and the PDA polymer backbones in the monolayer are responsible for the structured transfer of the striped PDA monolayer to the PDMS surface. Supplementary Figs. 4a,b illustrate the postulated structure of (a) PCDA and (b) dPE monolayers following transfer to PDMS via formation of such bonds. The inset in Supplementary Fig. 4a illustrates the torsion on the polymer backbone that would be created via local rehybridization at points where covalent linkages form, which may contribute to the observable fluorescence following transfer.

Solution-phase reactions were carried out utilizing dPE, and using 10,12-tricosadiynoic acid (TCDA), a 23-carbon analog of PCDA (25 carbons) that was available in the larger quantities required for the solution-phase reaction. Combining tricosadiynoic acid (TCDA) (Supplementary Fig. 4c) or dPE (Supplementary Fig. 4d) with triethylsilane (see Experimental Methods for details of reaction conditions) leads to formation of primarily disubstituted product for the TCDA reaction, and a mixture of mono- and di-substituted product for dPE, in addition to unreacted monomer. The very limited formation of trisubstituted product for the reaction with TCDA may be consistent with a higher degree of reactivity associated with alkynes in comparison with alkenes for this reaction. 


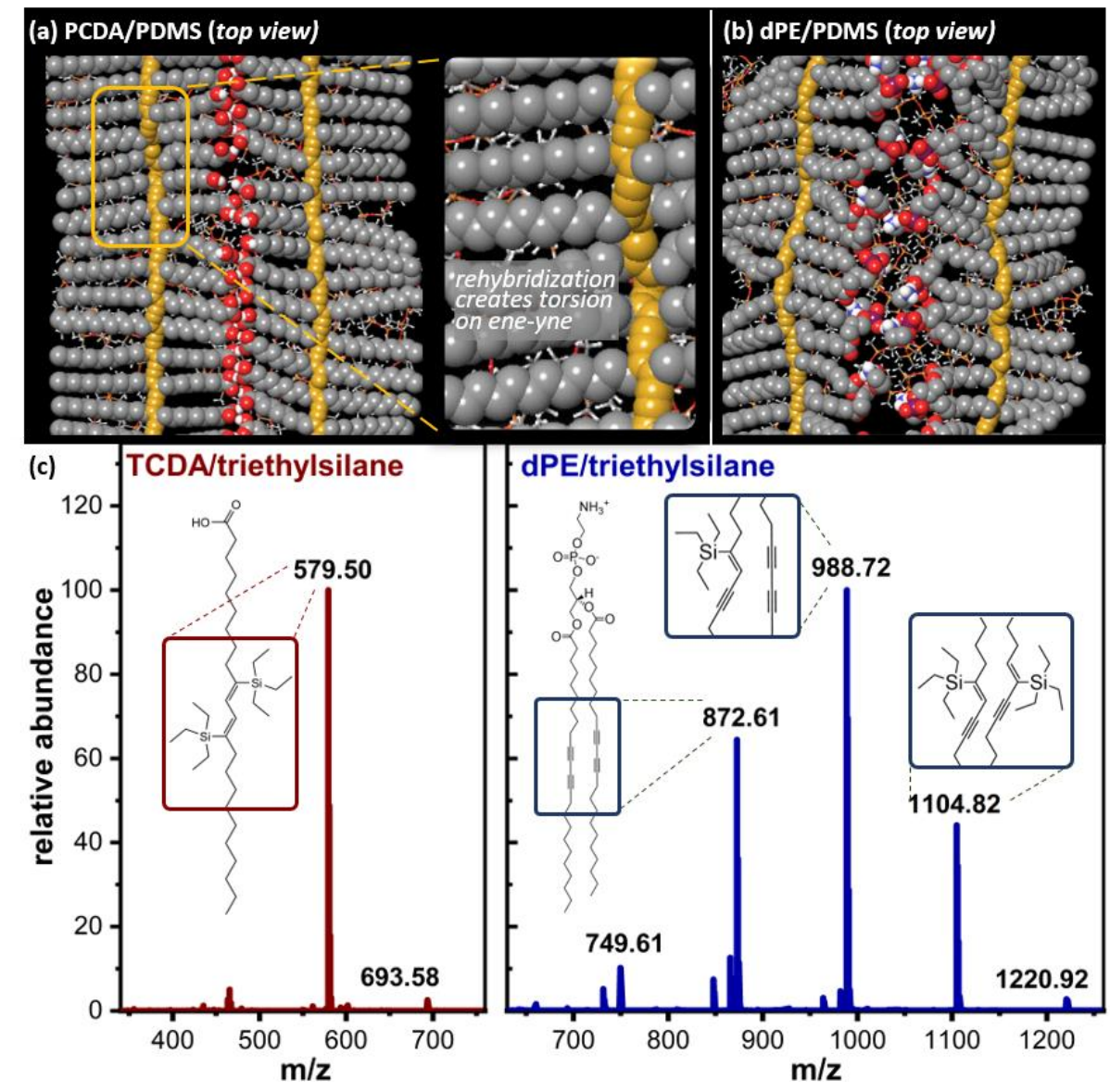

Figure 4. Molecular models of (a) PCDA/PDMS and (b) dPE/PDMS, illustrating torsion on the polymer backbone induced by the formation of covalent bonds to the PDMS. (c) Mass spectra of TCDA (left) and dPE (right) after exposure to triethylsilane, illustrating formation of primarily disubstituted reaction product for TCDA, and predominantly mono- and di-substituted product for dPE

\section{AFM images illustrating PDMS surface after transfer of striped phase films}

Although fluorescence imaging is the primary characterization modality employed to establish transferred monolayer properties in the main manuscript, the diffraction-limited spot size $(\sim 200 \mathrm{~nm})$ is large relative to the scale of individual polymers in the monolayer, so AFM images provide important nanoscale characterization of the smallest-scale transferred features.

When PDMS with a 10:1 base:crosslinker ratio is cured in contact with a bare HOPG surface and exfoliated (to provide the closest possible control for surfaces with monolayers transferred from HOPG), a network of pores is visible on the surface (Supplementary Fig. 5a,b). The measured average mesh size under these preparation conditions is $9.9 \pm 4.4 \mathrm{~nm}$, slightly smaller than previously reported persistence lengths for polydiacetylenes ( 16 nm). When the opposite face of the PDMS (which was exposed to air during the curing process) is imaged (Supplementary Fig. 5c), the pore network is less visible, though heterogeneity in the surface is visible on a similar length scale. 


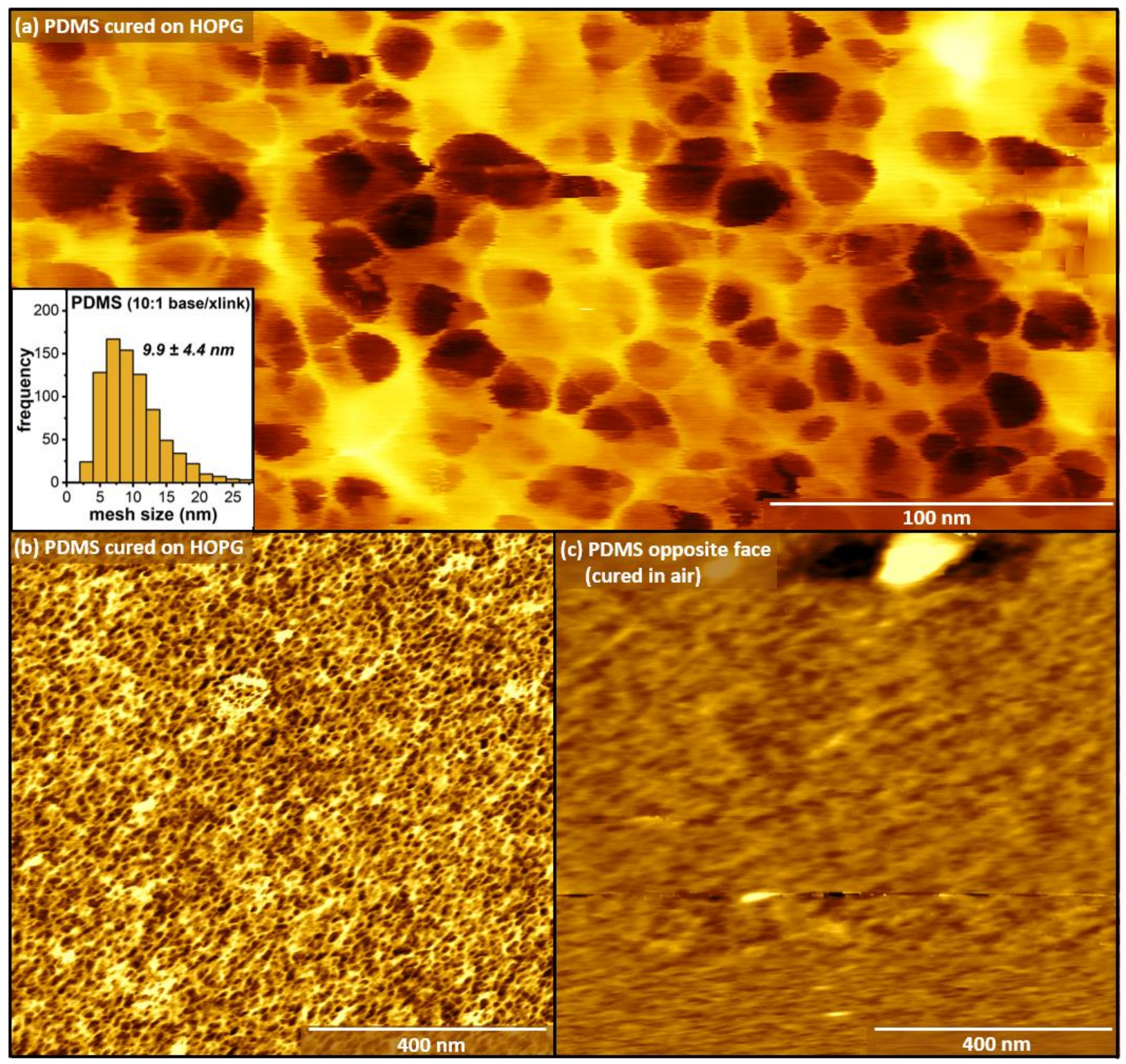

Figure 5. AFM images of bare PDMS surface (10:1 base:crosslinker ratio), illustrating surface pore structure. (a) and (b) show PDMS face cured in contact with HOPG; (c) shows opposing PDMS face cured in air. Inset in (a) shows histogram of mesh size distribution.

Following curing of PDMS on an HOPG surface functionalized with a striped monolayer and exfoliation of the PDMS from the HOPG surface, AFM images (Supplementary Fig. 6) reveal phase contrast differences consistent with structures commonly observed in the monolayers prior to transfer (such as Supplementary Figs. 1 and 2). In the AFM image below, acquired from a PDMS surface cured on a dPE monolayer, oval regions are morphologically consistent with vacancies observed in dPE monolayers on HOPG prior to transfer. Long, nearly parallel linear features throughout the image are qualitatively consistent with HOPG step edges commonly observed at this scale in AFM and SEM images prior to transfer.

AFM image quality is somewhat reduced in comparison with images of similar monolayers on harder HOPG surfaces, and we note that the monolayers are substantially more challenging to image than those on HOPG. Typical topographic features in the monolayer on HOPG are either stripes of headgroups ( 0.2 nm for dPE), molecular domain edges (0.3-0.6 nm, depending on domain structure), or HOPG step edges $(0.3 \mathrm{~nm})$. On the softer, amorphous PDMS surface, it is not always straightforward to resolve individual lamellae (as shown in Fig. 2 in the main manuscript). In cases in which lamellar resolution is possible, image features would be consistent with extended PDA backbones, pulled taut by periodic covalent linkages along the PDMS surface. 
Resolving monolayer structure at the domain (microscopic) scale is more straightforward. In the AFM phase image shown in Supplementary Fig. 6, below, edges of domains still appear consistent with domain morphologies prior to transfer, and surface coverage is qualitatively similar with that observed prior to transfer.

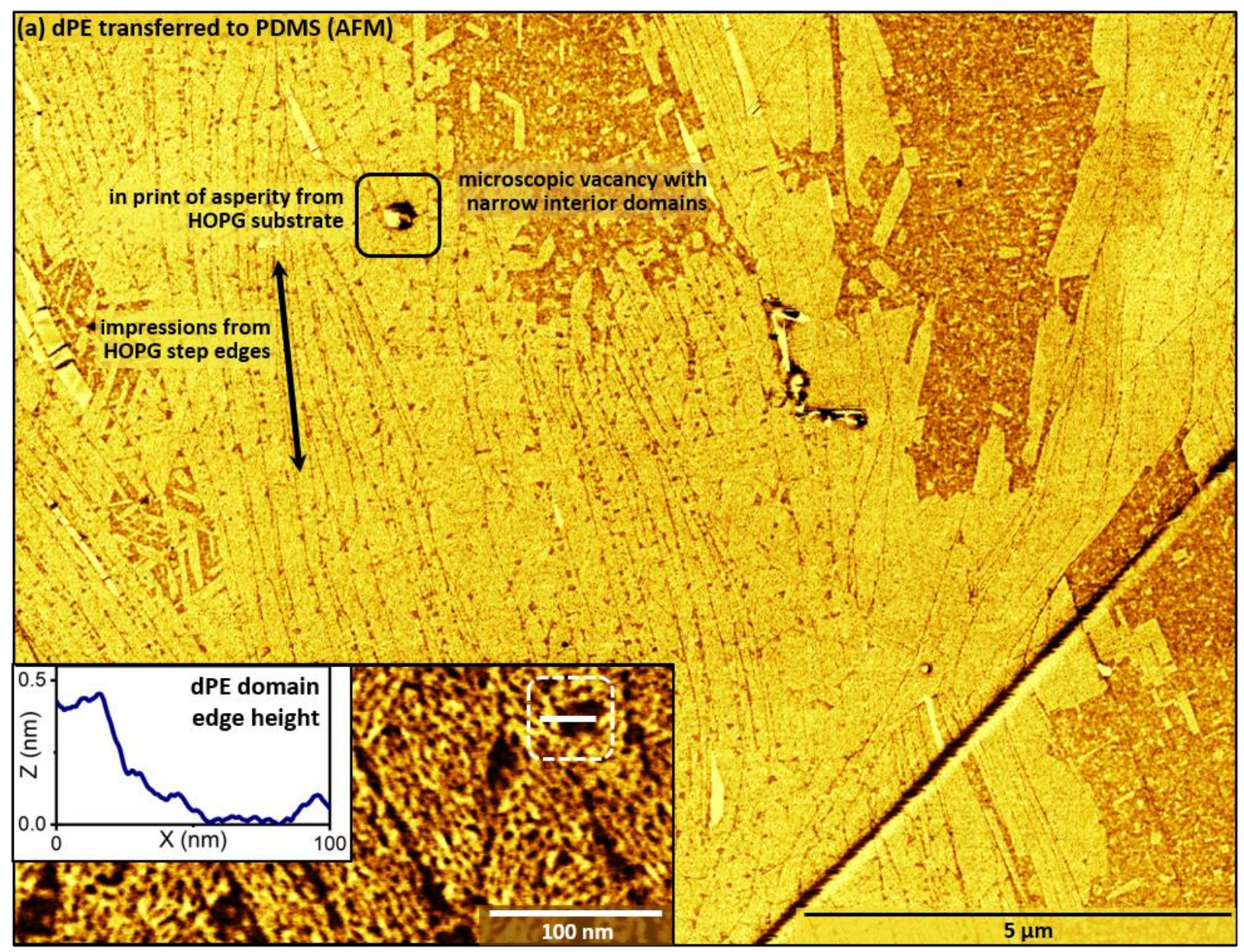

Figure 6. AFM image of dPE/PDMS, highlighting commonly observed features arising from monolayer and from substrate structure. Inset shows higher-resolution image of an area of the surface mostly covered with dPE domain. Line scan was acquired in the upper right corner of the image (highlighted with a white bar, enclosed with a box for emphasis), next to a small vacancy in the dPE domain, in order to illustrate measured topographic height difference between domain and bare PDMS.

\section{Fluorescence imaging of polydiacetylene monolayers on HOPG, and of HOPG on PDMS}

When striped PDA monolayers (such as that shown in Supplementary Fig. 7a,c,d) are transferred to PDMS and imaged by fluorescence (Supplementary Fig. 7b,e), sharp domain edges and morphologies are consistent with those observed in similar areas of films prior to transfer. In general, we do not perform fluorescence imaging in areas that have been imaged prior to transfer by SEM, since the SEM electron beam damages the monolayer during imaging, but image other areas of the surface. The widths of the smallest domains visible in Supplementary Fig. 7e $(\sim 200 \mathrm{~nm})$ are similar to domain structures observed on HOPG in Supplementary Fig. 7d, although exact domain widths at this scale would be difficult to resolve due to the diffractionlimited spot size. Fluorescence images shown in Supplementary Fig. 7 were collected using an Olympus BX-51 epifluorescence microscope with an $\mathrm{Hg}$ arc lamp source and a filter cube with an excitation band-pass filter from 460-490 $\mathrm{nm}$, a dichroic filter wavelength of $500 \mathrm{~nm}$ and a long-pass emission filter wavelength of $520 \mathrm{~nm}$. These imaging conditions produce a slightly more yellow tone in the detected image, in comparison with images collected with the confocal microscope. 


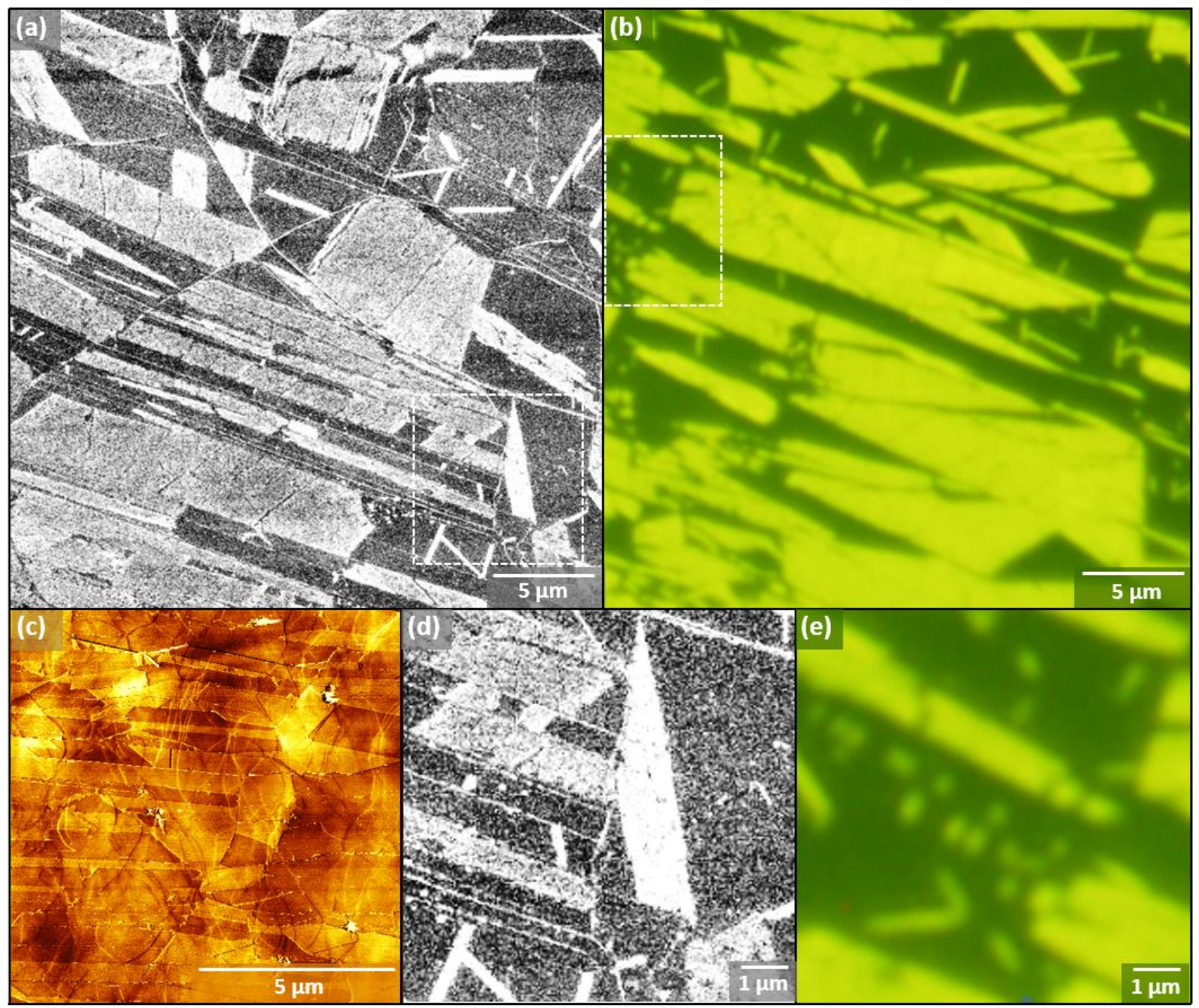

Figure 7. SEM and fluorescence images illustrating congruence of domain structure (in different areas of similarly structured surfaces), before and after transfer to PDMS. (a) SEM image of PCDA/HOPG and (b) fluorescence image of PCDA/PDMS. Different areas of surfaces exhibiting similar domain structures are utilized, since SEM imaging damages the monolayer structure. (c) AFM image of PCDA/HOPG, in an area also exhibiting similar domain structure; even at this scale, step edges and other topographic variations in the HOPG surface make it challenging to resolve monolayer domain structure. (d,e) Enlargements of region of $(a, b)$ highlighted with white boxes, showing domain structure at a similar scale to AFM image in (c). Smallest visible domains in both cases have widths $100-200 \mathrm{~nm}$.

Several images shown throughout the manuscript are collected utilizing a Zeiss upright confocal microscope with a 488-nm Ar laser for excitation, and a spectral detector with 8.9-nm bins, collecting with bin center values ranging from 495 to $691 \mathrm{~nm}$. Thus, images representing the full collected bandwidth (such as those in Supplementary Fig. 6) can be decomposed into a set of 23 images representing features that emit in each wavelength range. In Supplementary Fig. 8, for instance, a small graphite flake is visible as a bright feature in the 495-nm emission image, while contrast from features in the monolayer is maximized at wavelengths from 540 to $593 \mathrm{~nm}$. 


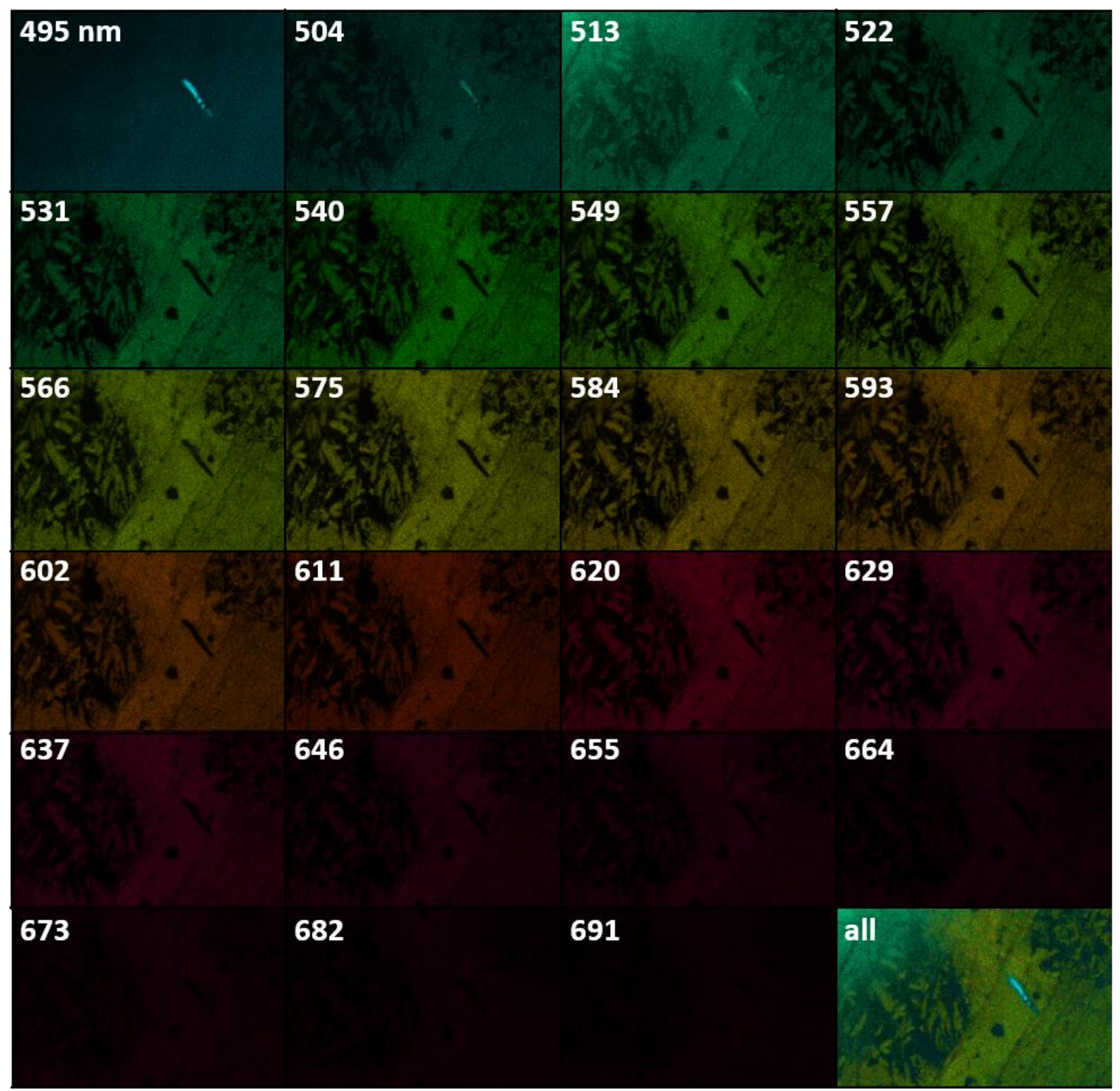

Figure 8. Images of the same region captured by the spectral detector in each wavelength range, followed by a composite image incorporating input from all bins.

Overall, micrographs of both dPE (Supplementary Fig. 9a) and PCDA (Supplementary Fig. 9b) transferred to PDMS reveal features congruent with those observed in SEM images of the monolayers on HOPG prior to transfer. In addition to the large regional contrast differences corresponding to vacancies in the monolayers, more subtle differences in local emission are observed within monolayers, in well-defined patterns, such as the microscopic stripes observed in the images of dPE in Supplementary Fig. 9a. Based on the length scale, morphology and the consistency of the bright/dark striped pattern, we suggest these are likely to arise from differences in local ordering of the alkyl chains in the dPE monolayer that impact polymerization and therefore fluorescence emission. 


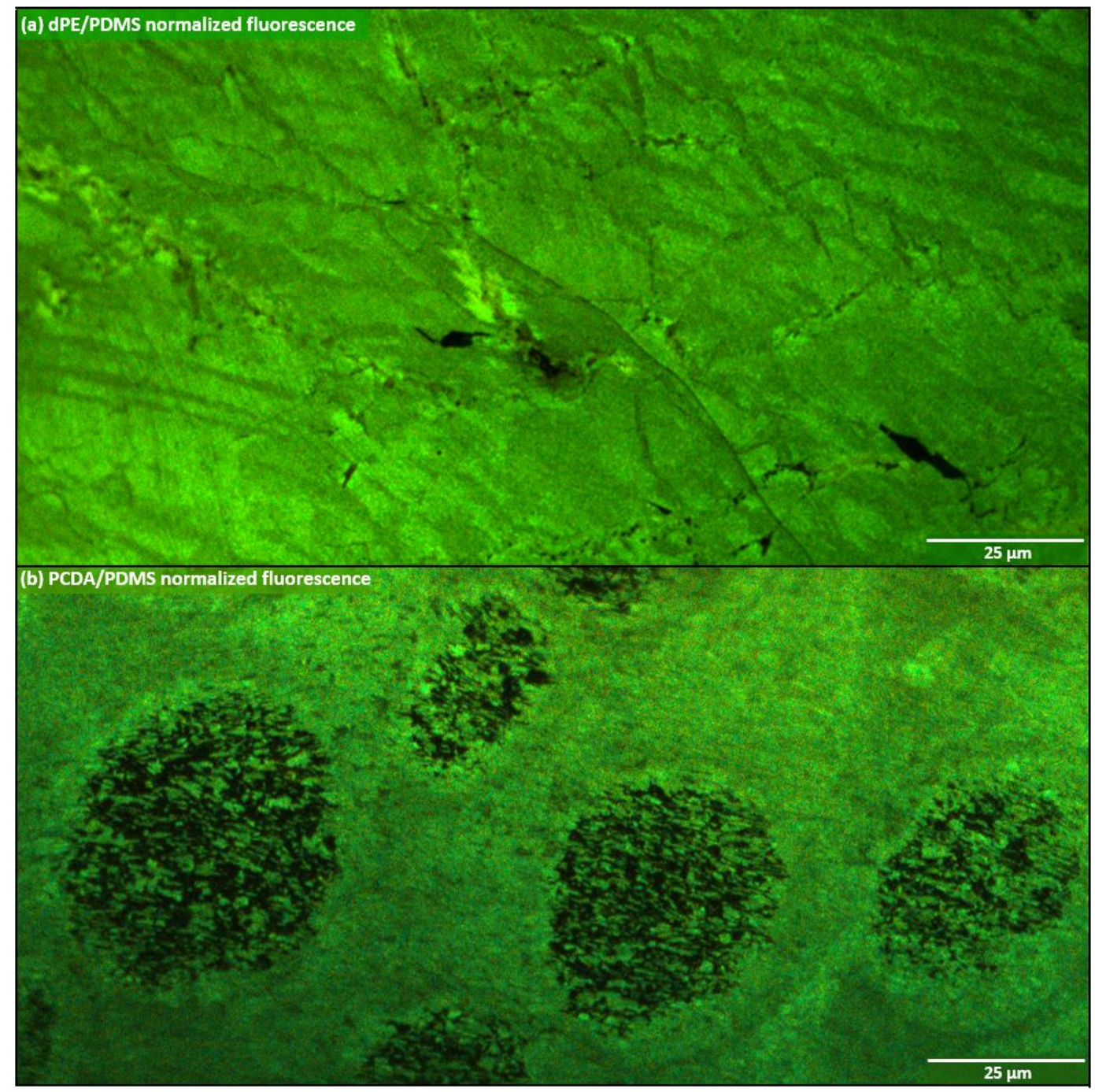

Figure 9. Fluorescence micrographs of (a) dPE/PDMS and (b) PCDA/PDMS, with imaging parameters selected to maximize contrast for each sample. PCDA monolayer in (b) was deposited on HOPG under conditions leading to large oval vacancies similar to those shown in Supplementary Fig. 1. dPE monolayer in (a) was deposited on HOPG under conditions that lead to nearly full coverage of striped phase.

To evaluate whether fluorescence imaging could also be utilized to characterize monolayer structure on HOPG prior to transfer (Supplementary Fig. 10a), we performed microscopy on dPE/HOPG (Supplementary Fig. 10b). Images were acquired using $\lambda_{\mathrm{exc}}=488 \mathrm{~nm}$ and a spectral detector collecting with bin center values from 495-691 nm with 8.9-nm bins (see Experimental Methods). In the image in Supplementary Fig. 10b, we observe small numbers of linear features that likely correspond to multi-layer step edges in the HOPG, as well as circular figures similar in appearance to topographical asperities in HOPG substrates (such as those in Supplementary Fig. 1). Spectra compiled based on specifying a region of interest and summing detected intensity in each wavelength range reveals a strong emission peak at $\sim 515 \mathrm{~nm}$ (Supplementary Fig. 10c). The inset in Supplementary Fig. 10c shows detected intensity in the wavelength range corresponding to dPE emission, to illustrate the absence of low-intensity peaks.

Supplementary Fig. 10d shows the emission image collected by the spectral detector in the bin centered at $549 \mathrm{~nm}$ (the emission maximum for dPE and PCDA in images on PDMS), which is featureless. The inset in Supplementary Fig. 10d shows the image collected under the parameters utilized to collect images of $\mathrm{PPE} / \mathrm{PDMS}$; the main images shows a larger area of the same sample at higher gain to illustrate the lack of obvious monolayer features in the collected emission. Overall, the lack of observed emission could reasonably result from formation of the non-emissive blue form of the PDA when polymerization is carried out on HOPG, or due to quenching of PDAs in contact with the HOPG substrate. 


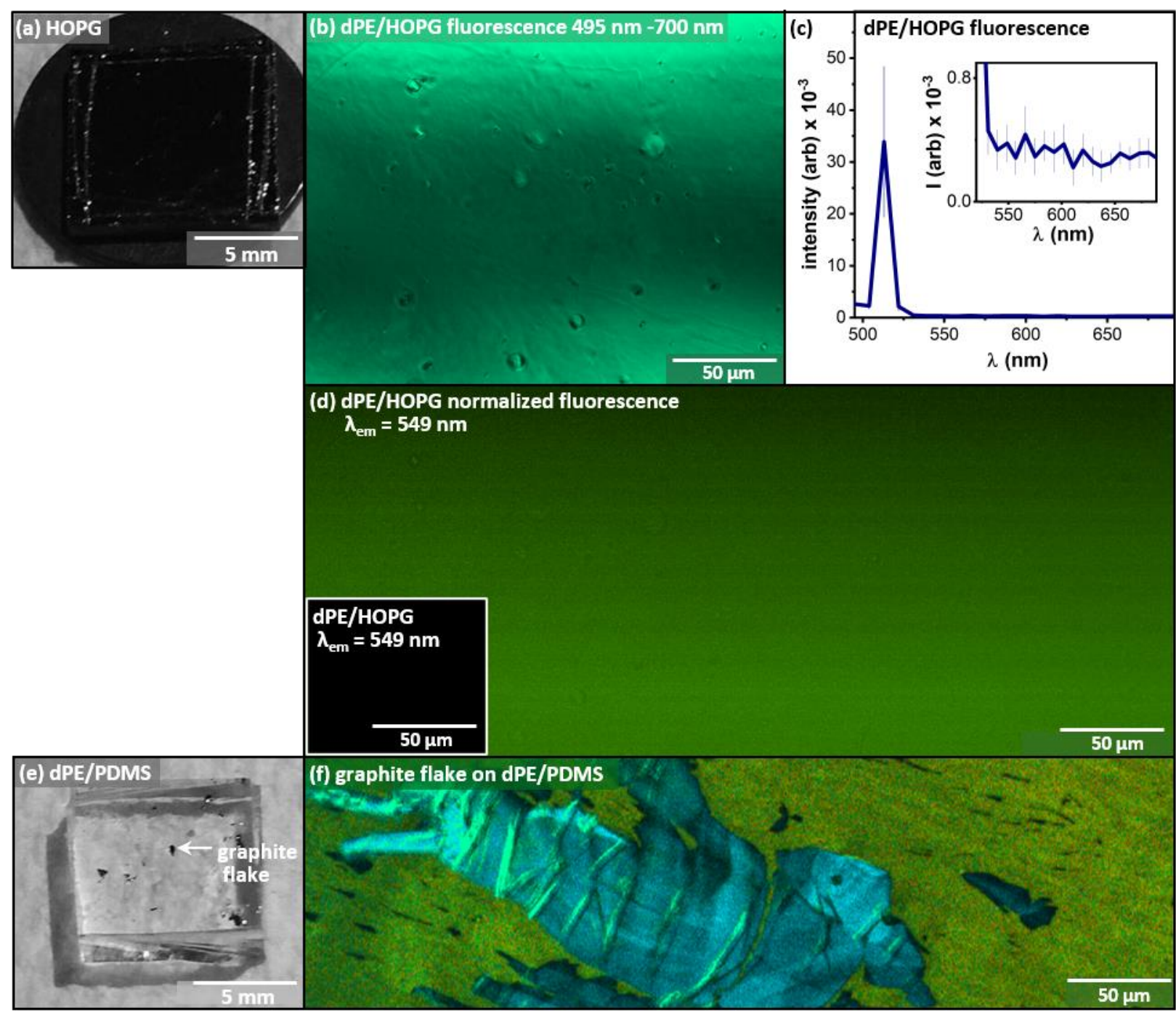

Figure 10. (a) dPE/HOPG, mounted on standard SEM puck. (b) Fluorescence micrograph of dPE/HOPG, with emission detected from 495-700 $\mathrm{nm}$. (c) Fluorescence spectrum of dPE/HOPG, illustrating primary peak at $\sim 515 \mathrm{~nm}$. Inset shows an enlargement of spectrum from 530-675 $\mathrm{nm}$, illustrating absence of polydiacetylene peaks. (d) Fluorescence micrograph of dPE/HOPG, with emission detected at $549 \mathrm{~nm}$ (emission maximum for dPE/PDMS). Inset shows detected emission utilizing settings used for collection of dPE images throughout manuscript; main image in (d) is normalized to illustrate lack of observable features. (d) Photograph of dPE/PDMS, illustrating presence of occasional small graphite flakes. (f) Fluorescence micrograph of dPE/PDMS, with graphite flake visible, showing difference in fluorescence emission in areas with graphite present.

Bare PDMS does not produce image or spectral features similar to those observed in transferred monolayers. Fluorescence spectra and images acquired on bare PDMS prepared in a process similar to that used for monolayer exfoliation (cured on bare HOPG then exfoliated, see Experimental Methods for detailed description) reveal a single peak at $\sim 515 \mathrm{~nm}$. Supplementary Fig. 11a shows spectra acquired over PDMS surfaces prepared using base:crosslinker ratios from 1:1 to 50:1 (the standard SYLGARD 184 preparation uses a 10:1 ratio). The peak increases in prominence with the amount of crosslinker present in the mixture. The inset in Supplementary Fig. 11a illustrates the absence of peaks in the region associated with features in polydiacetylene monolayers. Supplementary Fig. $11 \mathrm{~b}$ shows a fluorescence image including detected emission in the range from $495-700 \mathrm{~nm}$. Supplementary Fig. 11c shows only emission detected at $549 \mathrm{~nm}$, the emission maximum of the polydiacetylene monolayers. 

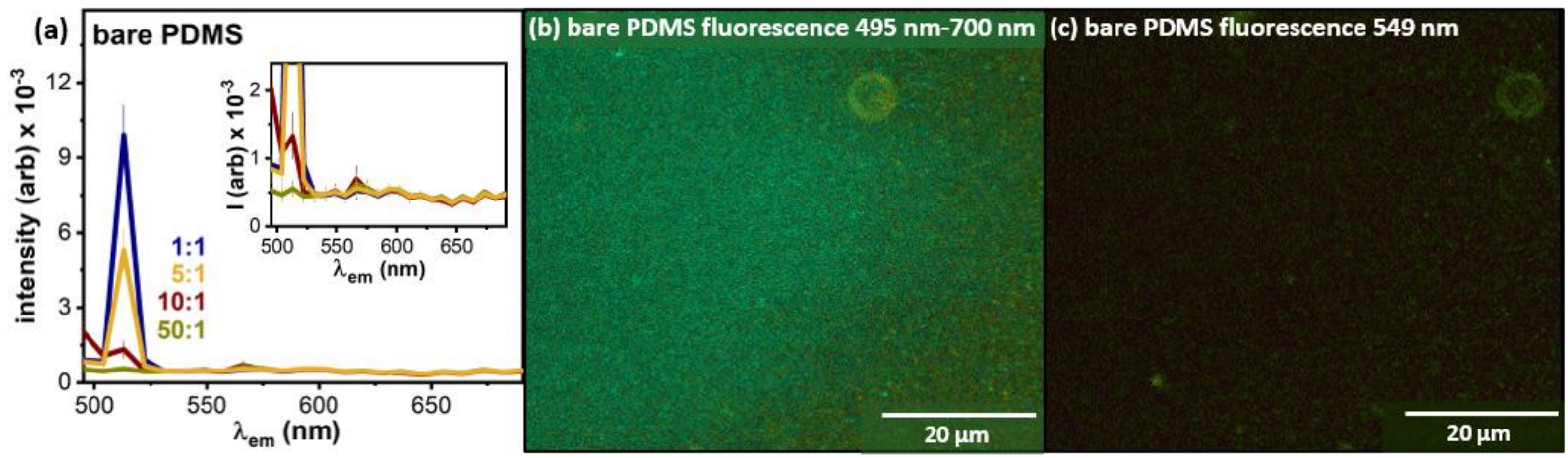

Figure 11. (a) Fluorescence emission spectrum for bare PDMS, compiled from pixels in a region of interest selected from a spectral imaging set. (b) Composite image of fluorescence emission from bare PDMS in the wavelength range from 495-700 nm. (c) Fluorescence emission of bare PDMS in the wavelength range corresponding to peak emission intensity for the PDA monolayers shown throughout the manuscript.

For $\mathrm{dPE} / \mathrm{PDMS}$ and PCDA/PDMS, fluorescence intensity in the PDA region of the spectrum also varies with the ratio of base:crosslinker in the PDMS. Supplementary Fig. 12 shows spectra for dPE (Supplementary Fig. 12a) and PCDA (Supplementary Fig. 12b) transferred to PDMS with base:crosslinker ratios from 1:1 to 50:1. Across the range of mixing ratios tested, monolayer fluorescence emission is greatest for the 10:1 base:crosslinker ratio (the same ratio recommended for standard use in the commercial SYLGARD 184 blend). Fluorescence micrographs from each series in Supplementary Fig. $12 a$ and $b$ are normalized against the fluorescence intensity of the monolayer transferred to 10:1 PDMS.

Fluorescence (and thus monolayer transfer) appears to be maximized at the base:crosslinker ratio designed to optimize network formation for the SYLGARD 184 PDMS blend. This finding would be consistent with the postulated transfer mechanism, in which one $\mathrm{Si}-\mathrm{H}$ bond in the crosslinker reacts with a polydiacetylene in the monolayer, and another $\mathrm{Si}-\mathrm{H}$ bond in the same crosslinker reacts with a vinyl group in the PDMS network. While mixing ratios other than 10:1 still cure the elastomer to produce a solid, it is reasonable that the use of a significant excess of crosslinker produces reactions with the monolayer that are unproductive for exfoliation (that is, the crosslinker reacts with the polydiacetylene, but does not also react with a vinyl group in a PDMS base polymer). Similarly, it is reasonable that a significant excess of vinyl groups from the PDMS base polymer would limit the number of crosslinker Si-H groups that form bonds to the polydiacetylene.

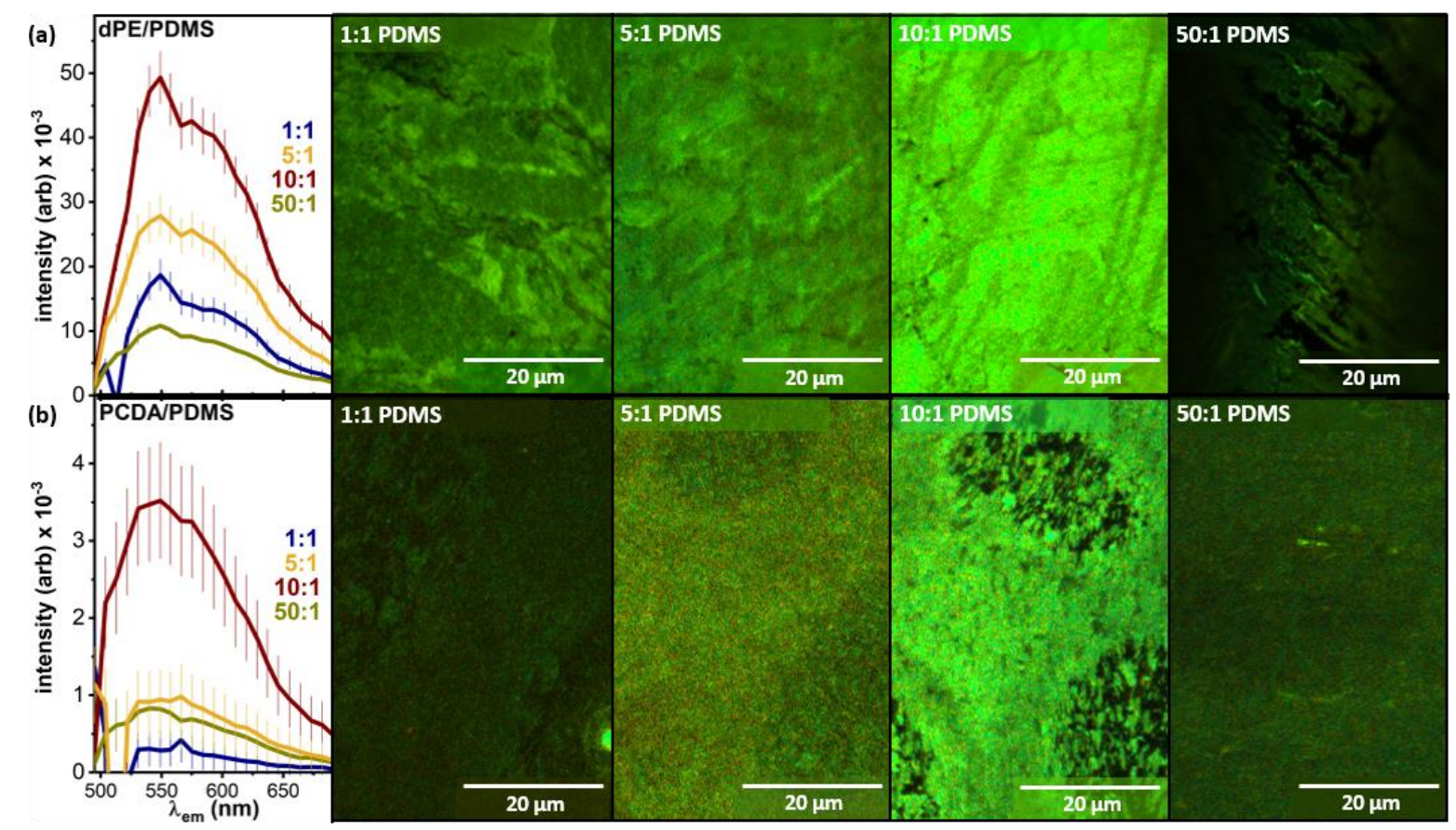

Figure 12. (a) Fluorescence emission spectrum and fluorescence micrographs for dPE/PDMS, with PDMS base:crosslinker ratios of 1:1, 5:1, 10:1, and 50:1. (b) Fluorescence emission spectrum and fluorescence micrographs for PCDA/PDMS, with PDMS base:crosslinker ratios of 1:1, $5: 1,10: 1$, and 50:1. Note difference in y axis maximum for the two sets of spectra. In each set of images, intensities are normalized against that of the 10:1 image for each series. 
We also examined whether it is necessary for the striped phase film to be polymerized prior to exposure to PDMS, in order for transfer to occur. Supplementary Fig. 13 compares spectra and images of PCDA/PDMS and dPE/PDMS films which have not been exposed to UV radiation (Supplementary Fig. 13a-e) with those that have been polymerized through UV irradiation (Supplementary Fig. 13f-h) prior to transfer. Fluorescence emission is approximately an order of magnitude lower for unpolymerized films (Supplementary Fig. 13a) in comparison with polymerized films (Supplementary Fig. 13f). Overall, images of PCDA/PDMS (Supplementary Fig. 13b,c) do not reveal morphological features characteristic of domain structures (such as Supplementary Fig. 13g). Images of dPE/PDMS (Supplementary Fig. 13d,e) reveal subtle intensity variations that are similar to those observed in polymerized dPE films after transfer (Supplementary Fig. 13h).

We rationalize this observed difference as follows. To create the domain structures used in the manuscript to illustrate transfer, PCDA is transferred near room temperature, while dPE is transferred using a heated transfer stage (set point temperature $70^{\circ} \mathrm{C}$ ). Diacetylenes are known to undergo thermal polymerization, ${ }^{18}$ and we believe that the observed $\mathrm{dPE}$ fluorescence in the film that was not subjected to UV irradiation prior to transfer may indicate a small amount of thermal polymerization (perhaps $10 \%$ of the final amount of polymerization) occurs during transfer.

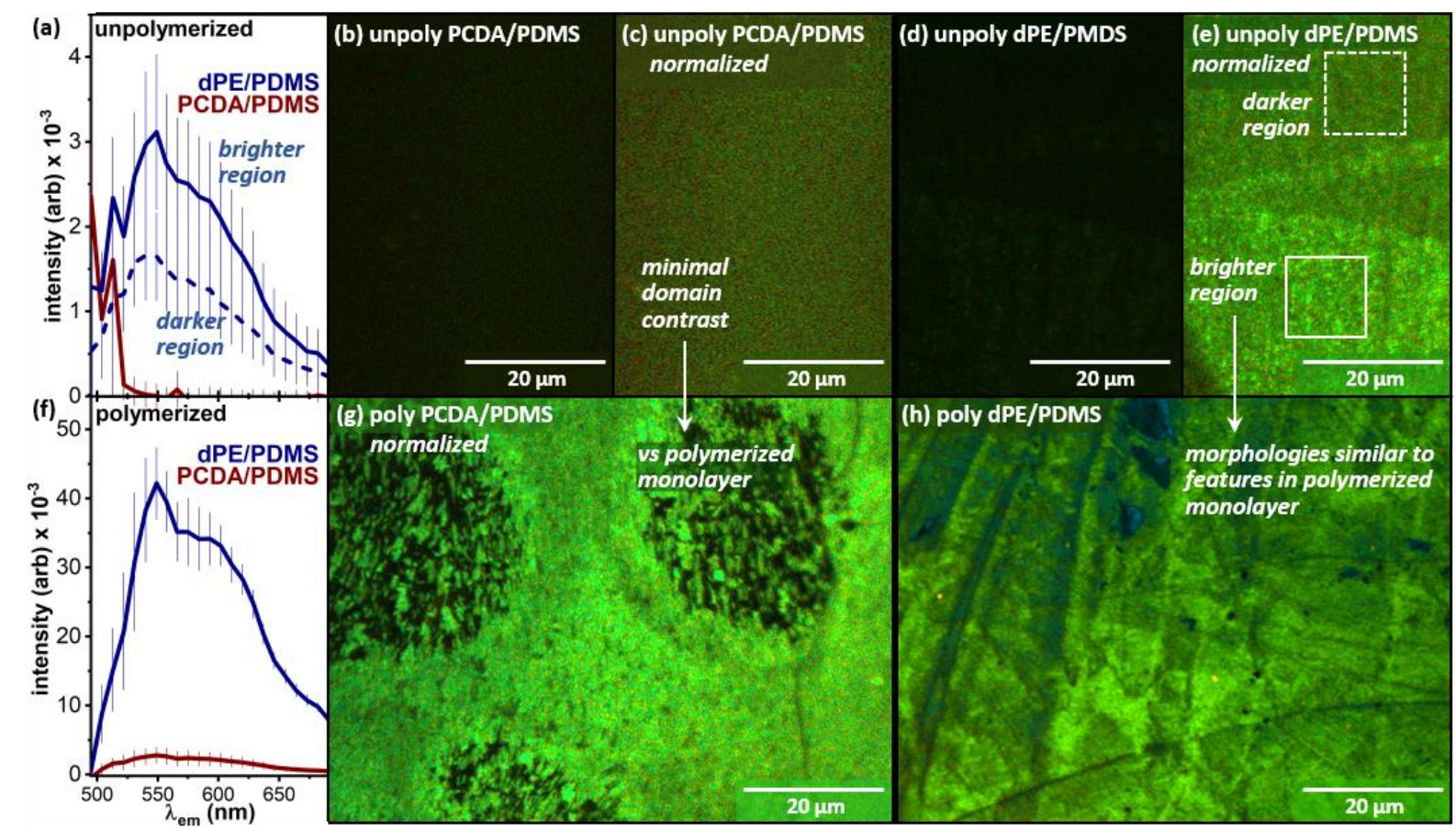

Figure 13. (a) Fluorescence emission spectra for unpolymerized dPE and PCDA transferred to PDMS. (b-e) Fluorescence emission images of (b,c) unpolymerized PCDA/PDMS, and (d,e) unpolymerized dPE/PDMS. Contrast for normalized images is selected to illustrate whether domain structure is visible within each image. ( $f-h)$ Fluorescence emission spectra $(f)$ and images (g,h) of polymerized dPE and PCDA for comparison with (a-e) above. Note difference in maximum values for two sets of spectra presented in (a) and (f).

Due to background fluorescence of the PDMS substrate, variations in local topography can result in variations in the balance of photon energies at the detector. Supplementary Fig. 14a shows a fluorescence image captured from a PCDA monolayer transferred to PDMS from an HOPG substrate with relatively tall step edges. Supplementary Fig. 14b illustrates a 3D AFM topography map (bottom) and a cartoon (top) relating the local surface height across the dashed line in Supplementary Fig. $14 \mathrm{a}$ with the height of the focal plane. In Supplementary Fig. 14c, an image of the same area of the surface is shown with the bin centered at $515 \mathrm{~nm}$ (associated with PDMS fluorescence) subtracted from the image. Supplementary Fig. 14d shows spectra acquired from the three areas highlighted with white boxes in Supplementary Fig. 14a. 


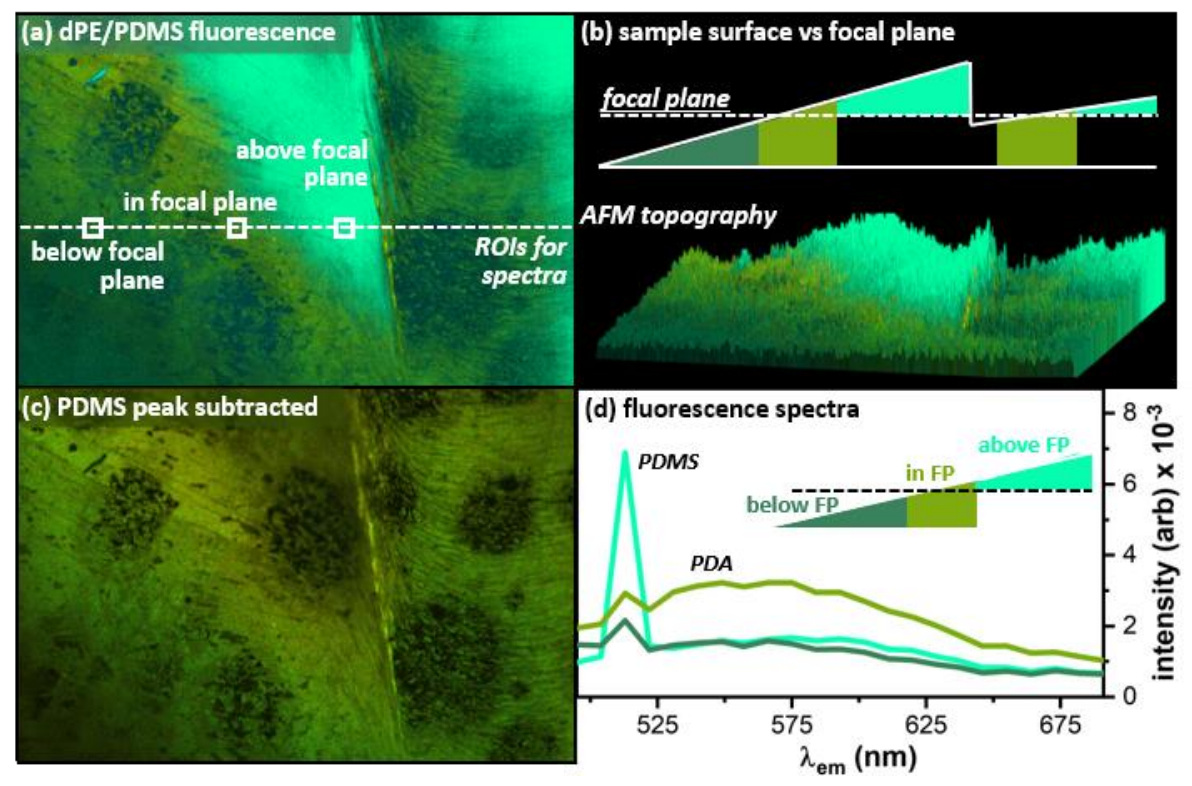

Figure 14. (a) Fluorescence image of dPE/PDMS with a stepped surface due to step edges in HOPG substrate used for transfer. (b) Cartoon (top) and AFM topography (bottom) illustrating height at points across the surface. Schematic illustrates topography across profile shown with dotted line in panel (a), and highlights color changes as surface height changes vs focal plane. (c) Composite fluorescence image of area shown in (a) with $515 \mathrm{~nm}$ image channel subtracted. (d) fluorescence spectra from three areas in (a) highlighted with white boxes.

\section{Fluorescence imaging of mixed monolayer}

In the main manuscript, Fig. 3, a small region of a fluorescence image of a mixed monolayer of PCDA and dPE on PDMS is shown, to illustrate that differences in fluorescence intensity associated with the two molecules can be used to distinguish areas of the surface functionalized with the two chemistries. In Supplementary Fig. 15, we show a larger region of the surface, for visual inspection.

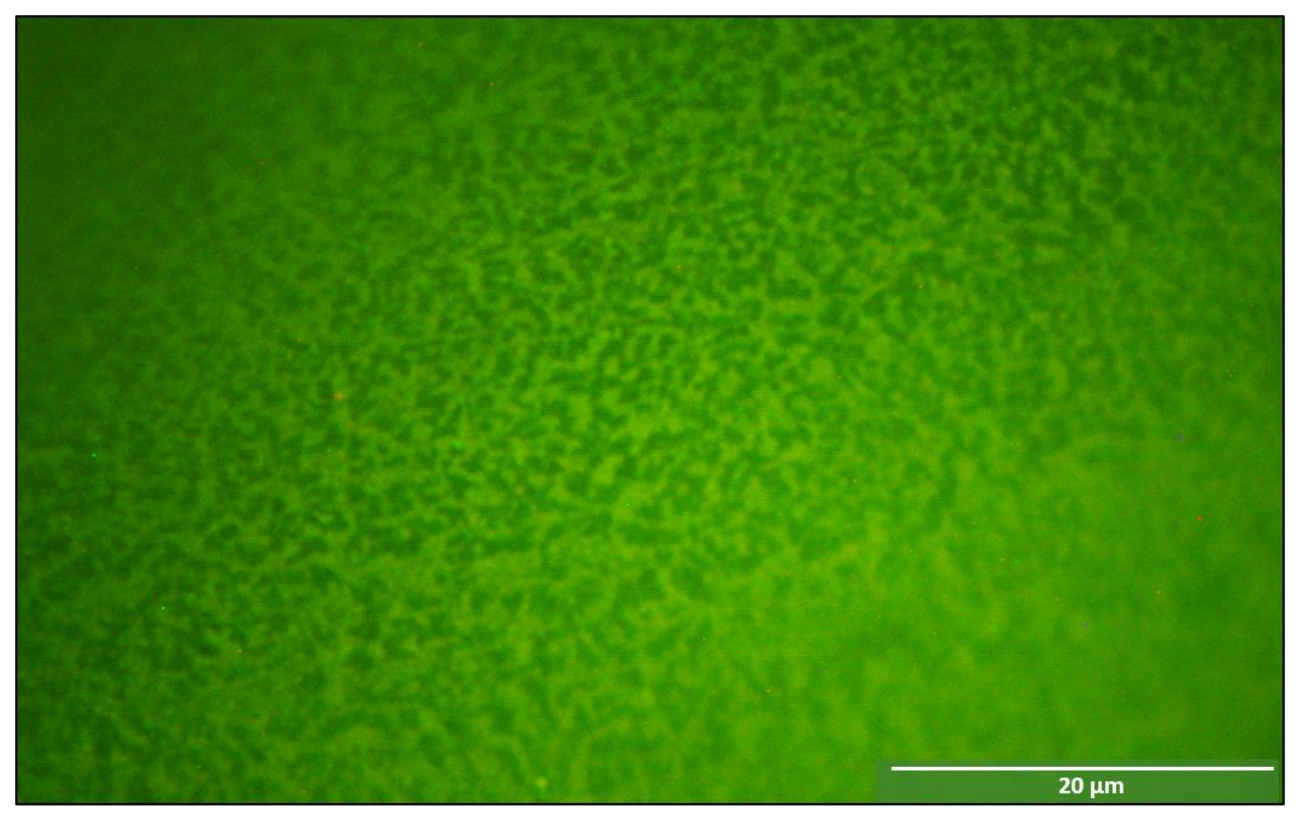

Figure 15. Fluorescence micrograph of a mixed monolayer of dPE and PCDA transferred to PDMS. 


\section{Polarized fluorescence imaging of dPE/PDMS}

As described in the main manuscript, polydiacetylene fluorescence emission is known to be strongly polarized along the polymer axis; we utilized this property to provide information about local polymer orientation in the transferred films, as shown in the main manuscript, Fig. 4. For these measurements, we assembled dPE on $\mathrm{MoS}_{2}$, which we have observed previously can produce very long, narrow domains ${ }^{19}$ that in this case were anticipated to produced narrow bands of highly polarized emission alternating with darker areas (vacancies) between the domains. The main manuscript shows a small area of such an image, first with unpolarized emission, and then two images with the emission channel polarized along one of two lamellar alignment directions.

Supplementary Figs. 16 and 17 show SEM images of this dPE assembly morphology on $\mathrm{MoS}_{2}$, with narrow domains with lengths up to $\sim 10 \mu \mathrm{m}$ oriented along crystallographically equivalent directions of the MoS 2 substrate. Supplementary Fig. 18 shows a larger area of the dPE/PDMS surface from which images in Fig. 4 were extracted. Supplementary Fig. 18a shows an unpolarized image, while the remaining subpanels show a series of polarized images of the same area of the surface (polarization axis indicated with a white arrow); the polarizer is rotated $15^{\circ}$ after acquisition of each image. For scale, the SEM image in Supplemental Fig. 16 is similar in area to that shown in each subpanel of Supplementary Fig. 18, while the edge length in Supplementary Fig. 17 is similar to that of the scale bar in each subpanel of Supplementary Fig. 18.

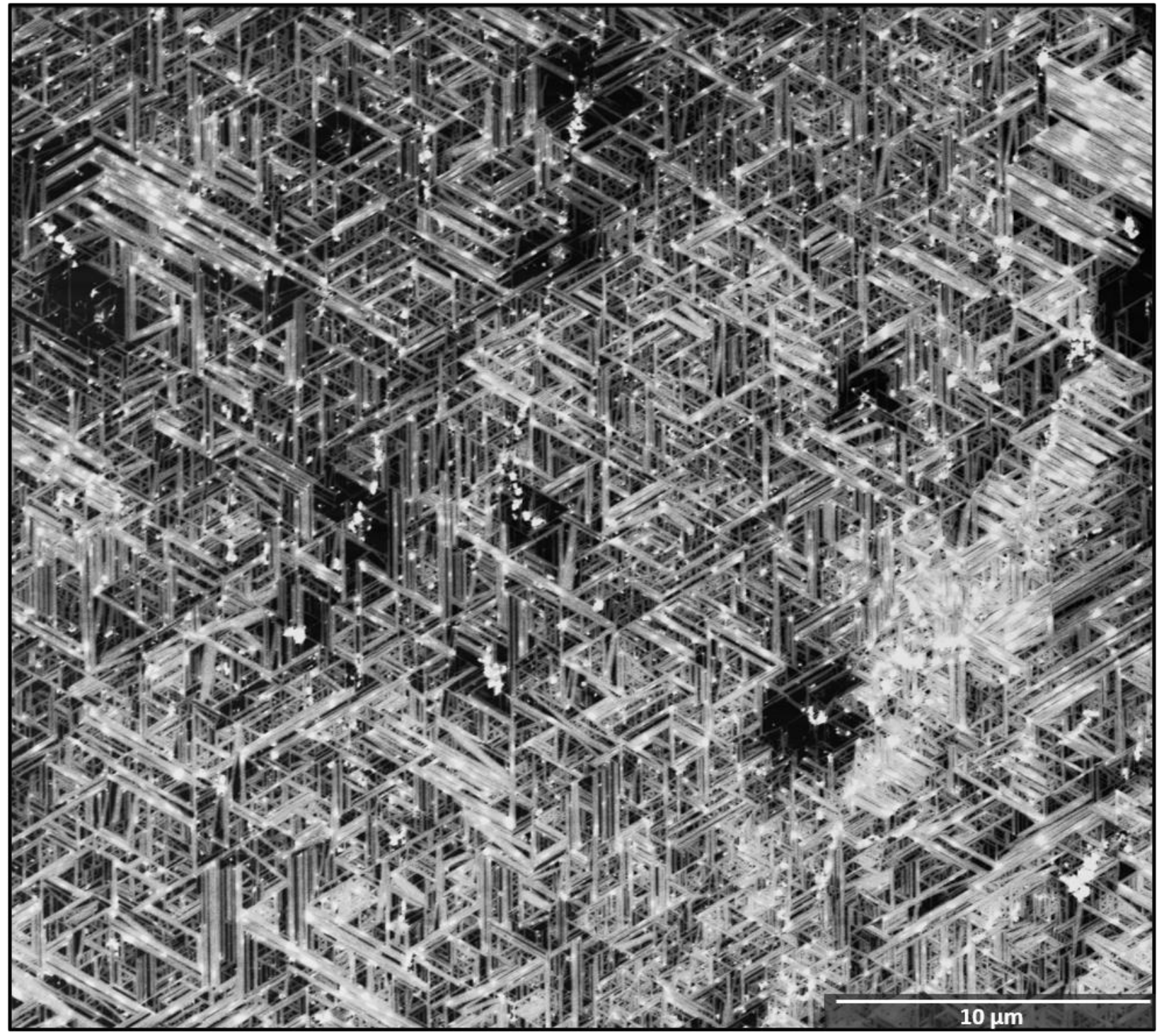

Figure 16. SEM image of dPE assembled on $\mathrm{MoS}_{2}$ via LS conversion, producing long ordered molecular domains suitable for polarized fluorescence imaging. Here, closely spaced narrow domains are oriented along all equivalent crystallographic axes of the $\mathrm{MoS}_{2}$ substrate. Image area is similar in size to image area in each subpanel of Supplementary Fig. 18. 


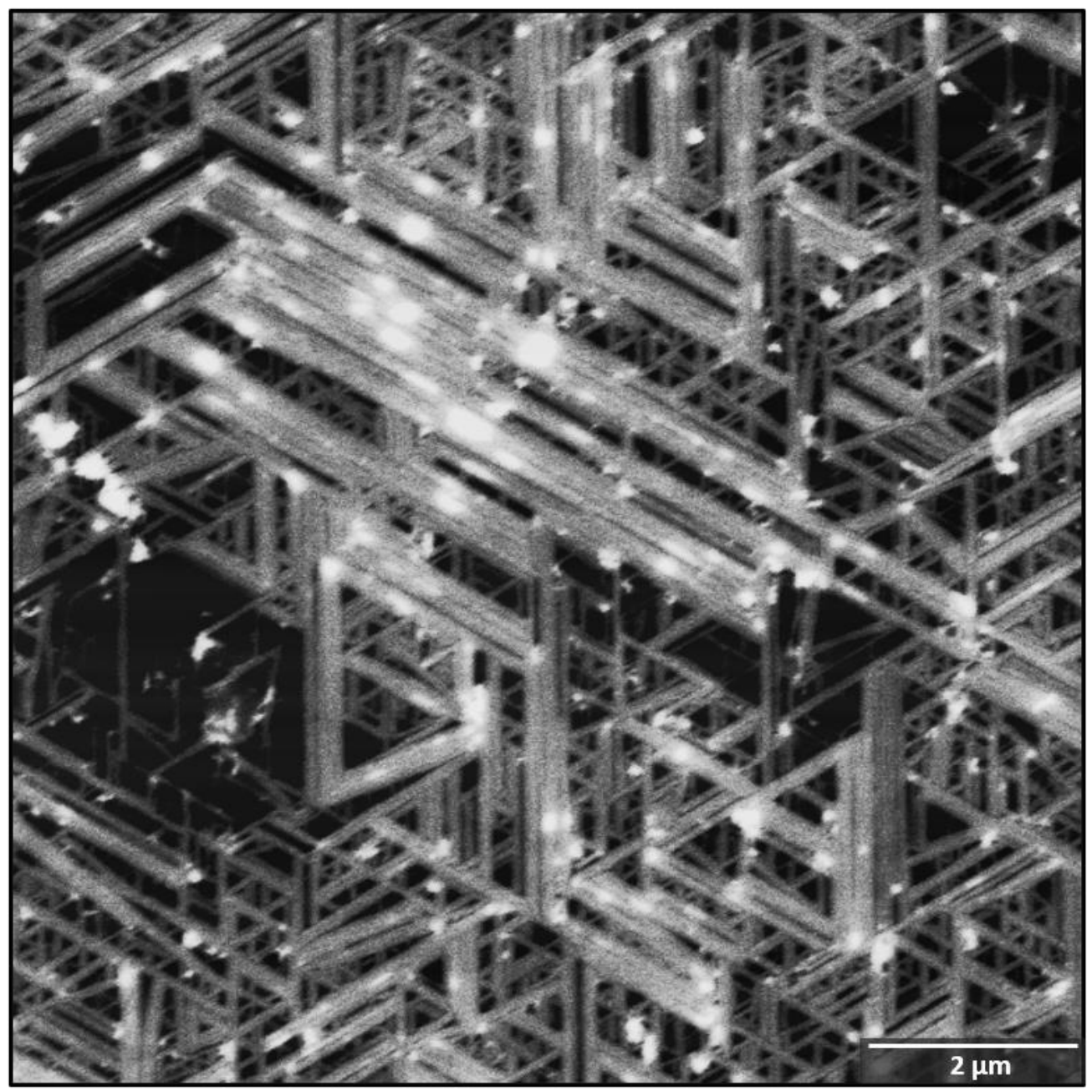

Figure 17. Enlargement of a small area of the SEM image in Supplementary Fig. 16, illustrating more detail of domain orientation. Image edge length here is similar to scale bar length in subpanels of Supplementary Fig. 18. 


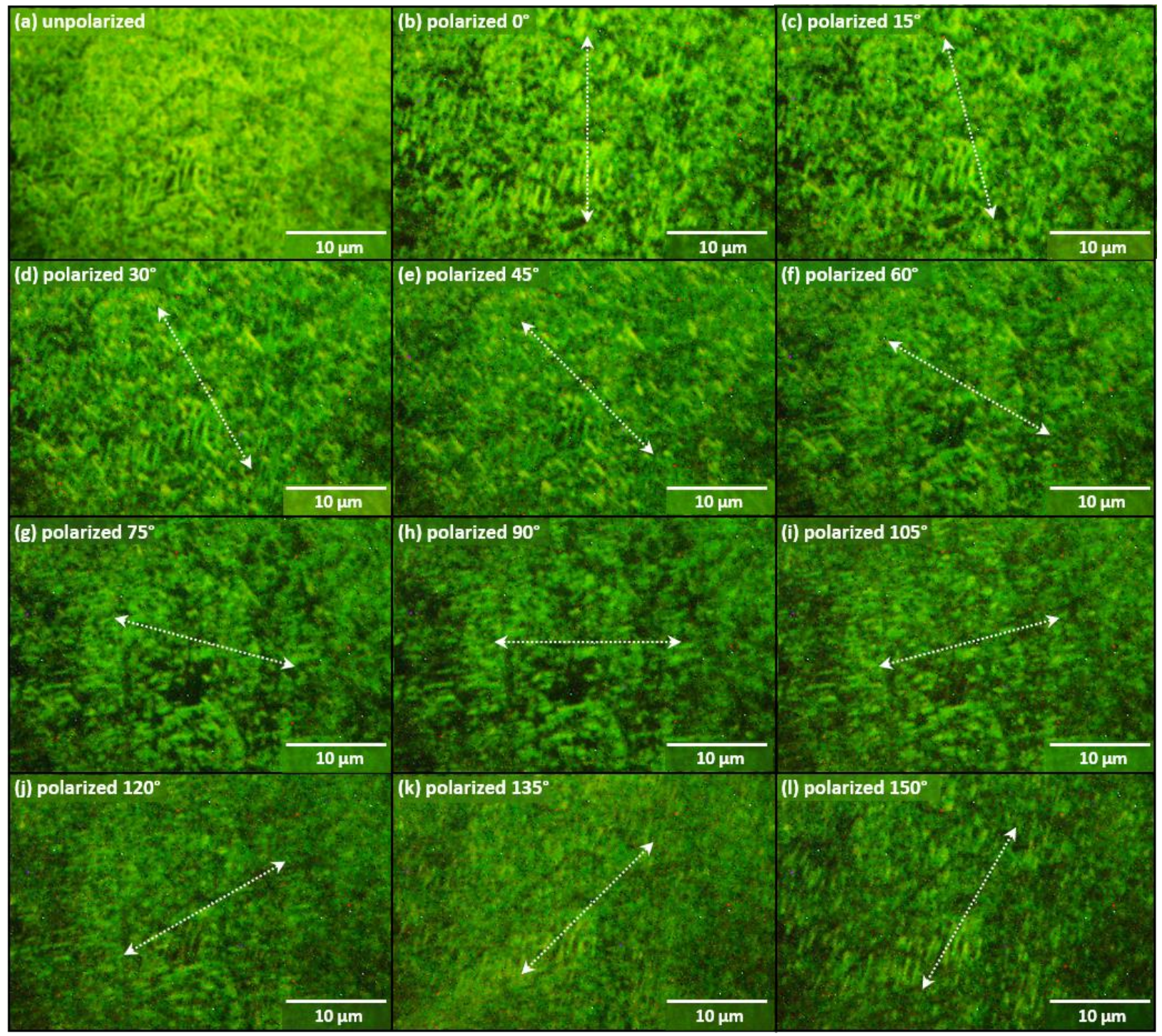

Figure 18. Fluorescence micrographs of dPE/PDMS, in an area with lamellae (polydiacetylenes) oriented along the three crystallographically equivalent axes of the original assembly substrate $\left(\mathrm{MoS}_{2}\right)$. The series of images were captured in the same area, sequentially: (a) with unpolarized emission, and (b-l) with emission polarized along the direction shown in the arrow. 
Assembly of dPE on $\mathrm{MoS}_{2}$ can also produce areas with a primary alignment direction such as those shown in Supplementary Figs. 19 (SEM) and 20 (fluorescence). Supplementary Fig. 20 shows a large area of a dPE surface with a primary alignment direction (again, the unpolarized image is shown in Supplementary Fig. 20a), followed by a series of images with the polarizer rotated $15^{\circ}$ prior to each sequential image acquisition. The images with the highest degree of alignment between the lamellar (polydiacetylene) axis and the polarizer are intentionally collected last in the sequence, to illustrate that the polarized contrast is still visible after several images are collected in the same region.

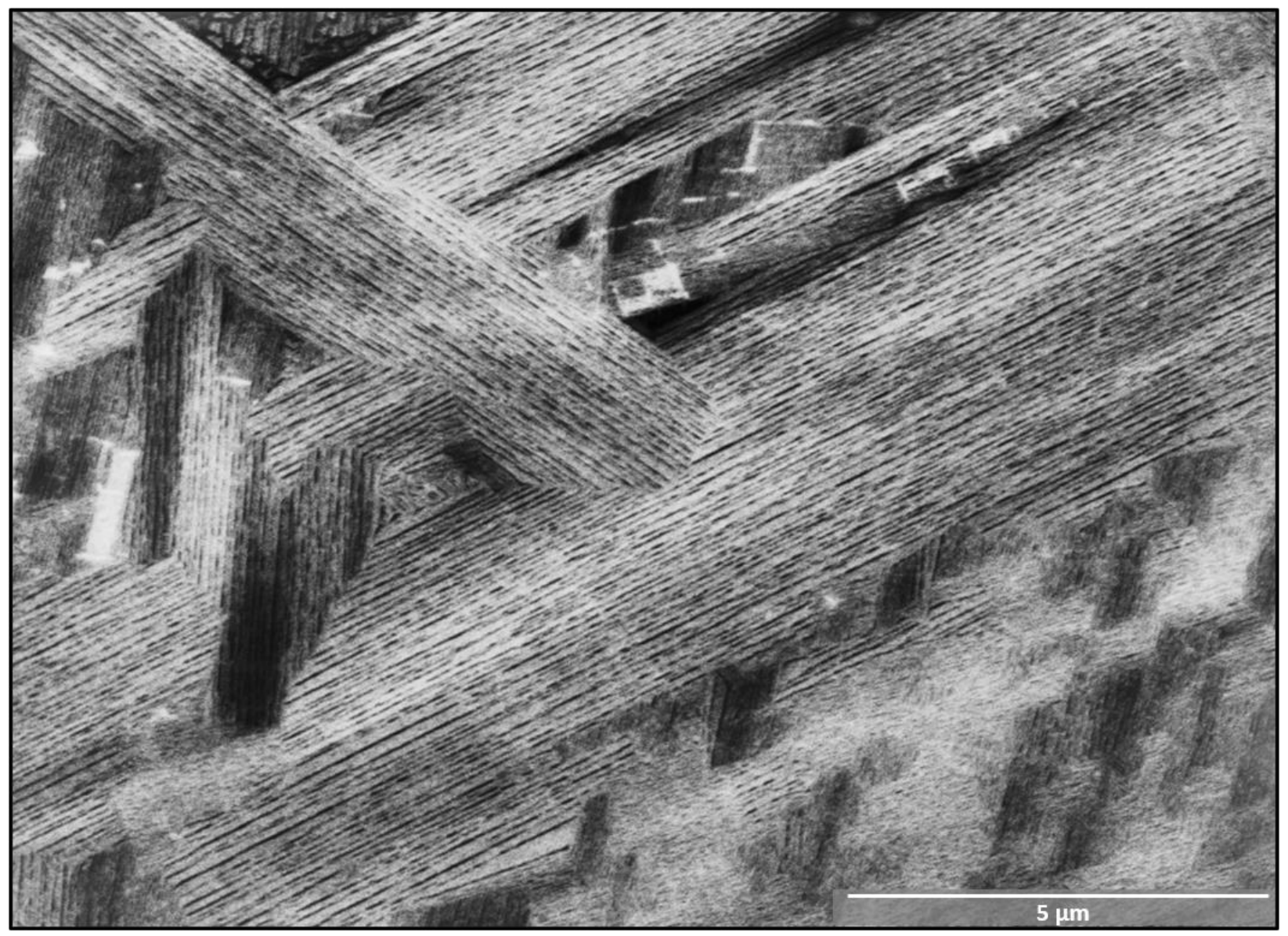

Figure 19. SEM image of dPE assembled on $\mathrm{MoS}_{2}$ via LS conversion, producing long ordered molecular domains suitable for polarized fluorescence imaging. 


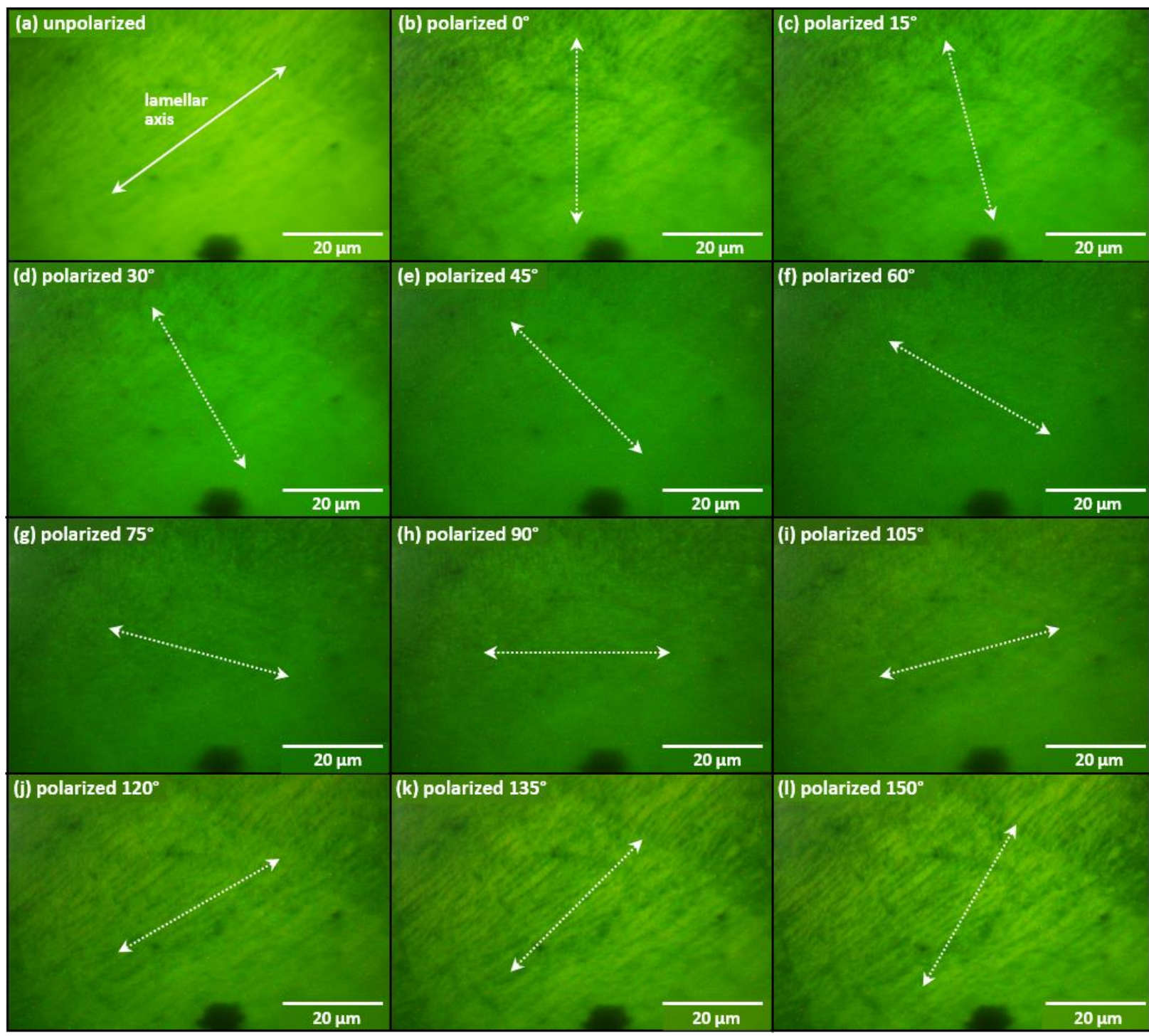

Figure 20. Fluorescence micrographs of dPE/PDMS, in an area with one predominant lamellar (polymer) orientation. The series of images were captured in the same area, sequentially: (a) with unpolarized emission, and (b-l) with nominal emission polarization axis highlighted by the arrow. Arrow in (a) highlights the main direction of the lamellar axes in the area shown, to illustrate domain contrast emerges when the polarizer axis is nearly parallel to the lamellar axis. 


\section{Fluorescence emission spectrum of $I_{2}$}

In the main manuscript, we expose polydiacetylene monolayers to $I_{2}$, which has been used previously as an approach modify the electronic structure of the conjugated backbone in standing phases of polydiacetylenes. Here (Supplementary Fig. 21 ), we show the fluorescence emission spectrum of $I_{2}$ for comparison with spectra of $I_{2}$-treated monolayers presented in the main manuscript (Fig. 4). Here, in Supplementary Fig. 22, we also show unmodified (Supplementary Fig. 22a) and $\mathrm{I}_{2}$-exposed (Supplementary Fig. 22b) PCDA monolayers prepared using LS conversion.

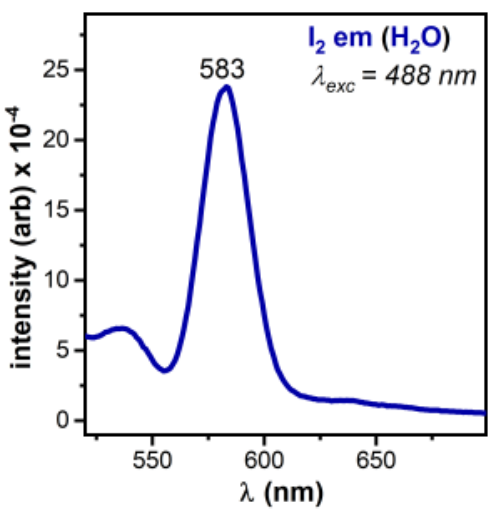

Figure 21. Fluorescence emission spectrum of $\mathrm{I}_{2}$ in $\mathrm{H}_{2} \mathrm{O}$, with $\lambda_{\mathrm{exc}}=488 \mathrm{~nm}$ (excitation wavelength used for PCDA/dPE imaging in manuscript).

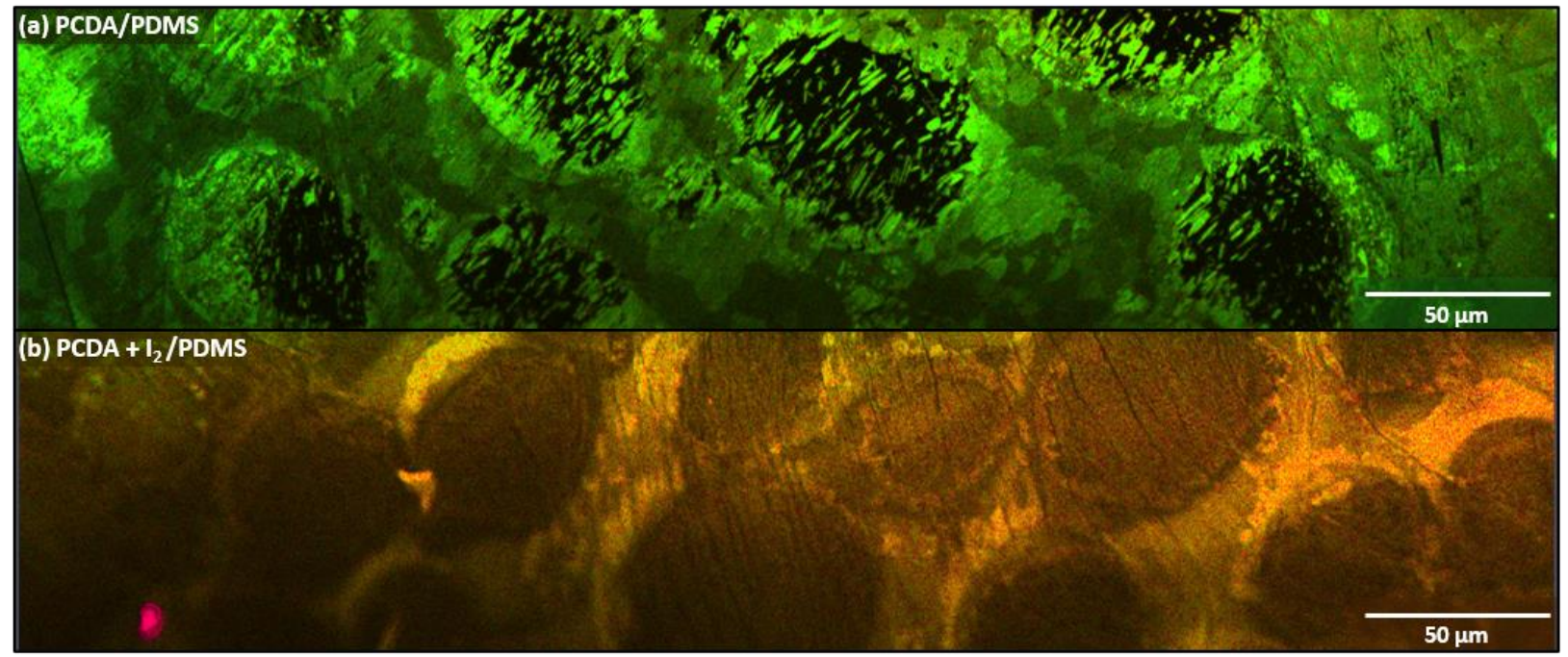

Figure 22. Fluorescence images of PCDA/PDMS (a) without, and (b) with exposure to $I_{2}$ prior to transfer. 


\section{Fluorescence images of PCDA/PDMS and dPE/PDMS before and after exposure to divalent ions}

In the main manuscript we expose PCDA/PDMS and dPE/PDMS to buffers containing divalent ions and monitor increases in fluorescence intensity. Here (Supplementary Figs. 23-25), we show images before and after exposure to $\mathrm{CaCl}_{2}$ or $\mathrm{CdCl}_{2}$ with normalized intensity, to illustrate that although fluorescence intensity changes, there are not substantial structural changes to microscopic monolayer structure.

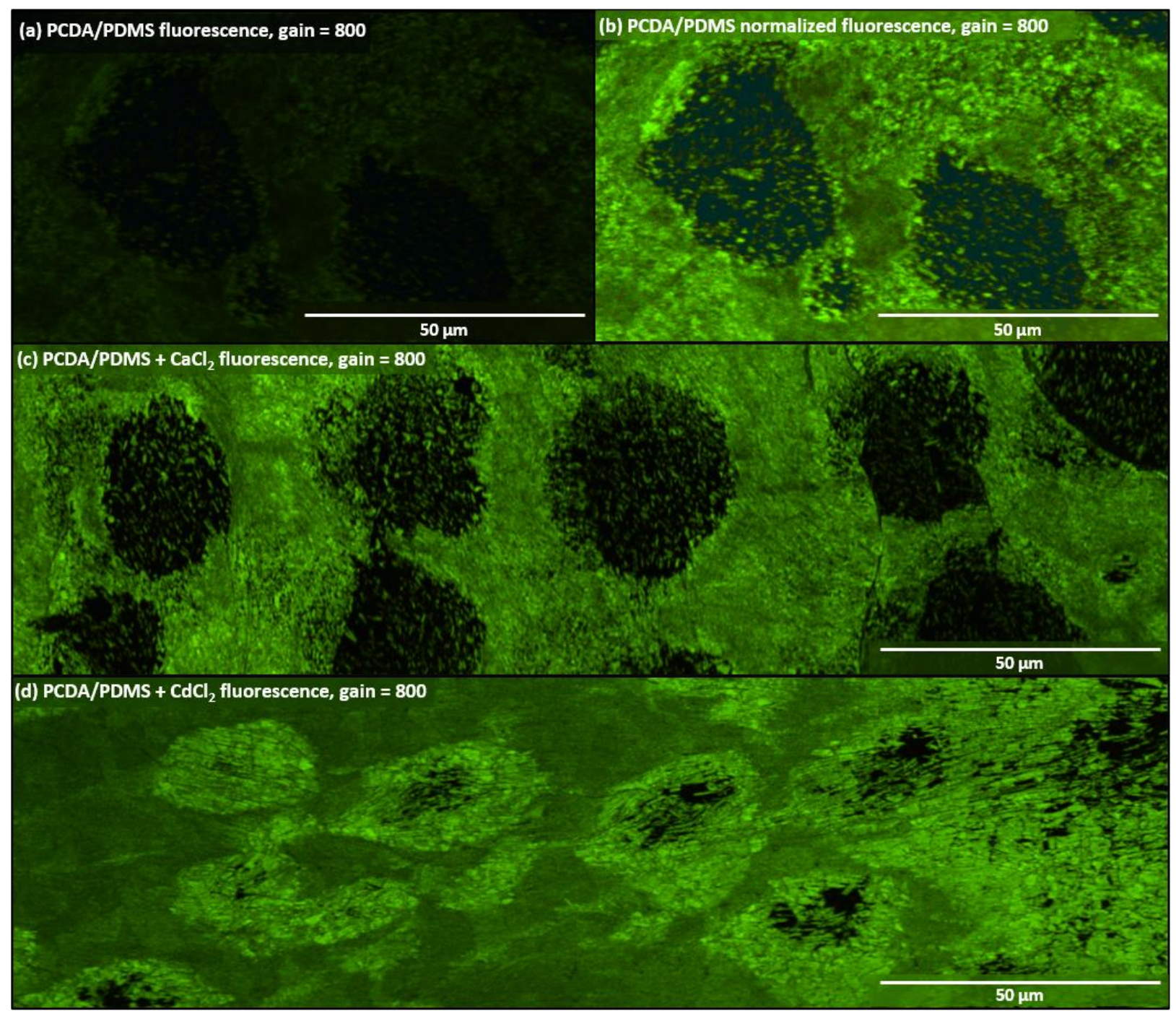

Figure 23. Fluorescence micrographs of PCDA/PDMS (a) under standard imaging conditions, (b) same image with contrast adjusted so that monolayer features are visible, and after exposure to (c) $100 \mathrm{mM} \mathrm{CaCl}_{2}$ buffer or (d) $100 \mathrm{mM} \mathrm{CdCl}_{2}$ buffer. 


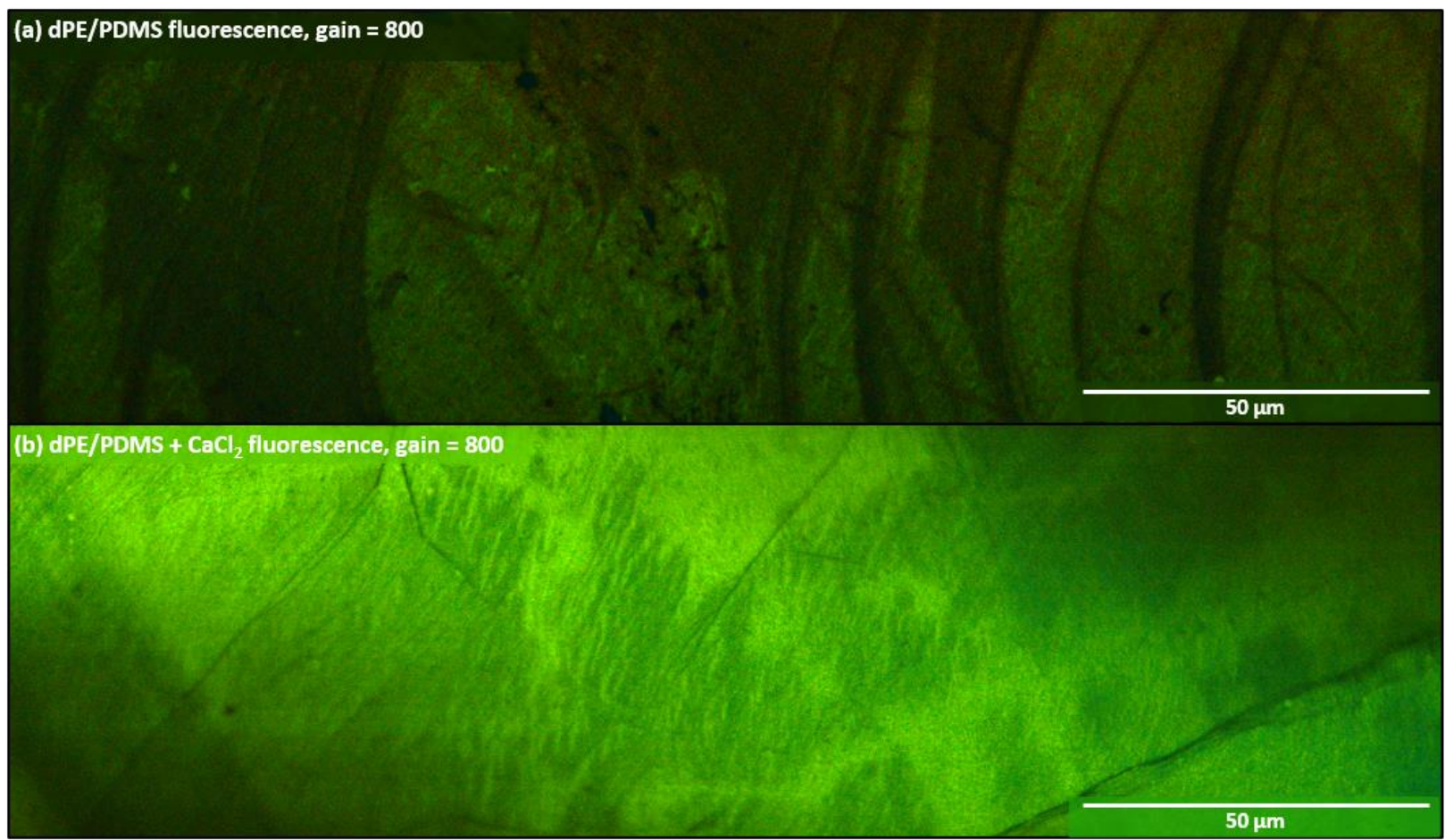

Figure 24. Fluorescence micrographs of dPE/PDMS (a) under standard imaging conditions, and (b) after exposure to $100 \mathrm{mM} \mathrm{CaCl}_{2}$ buffer.

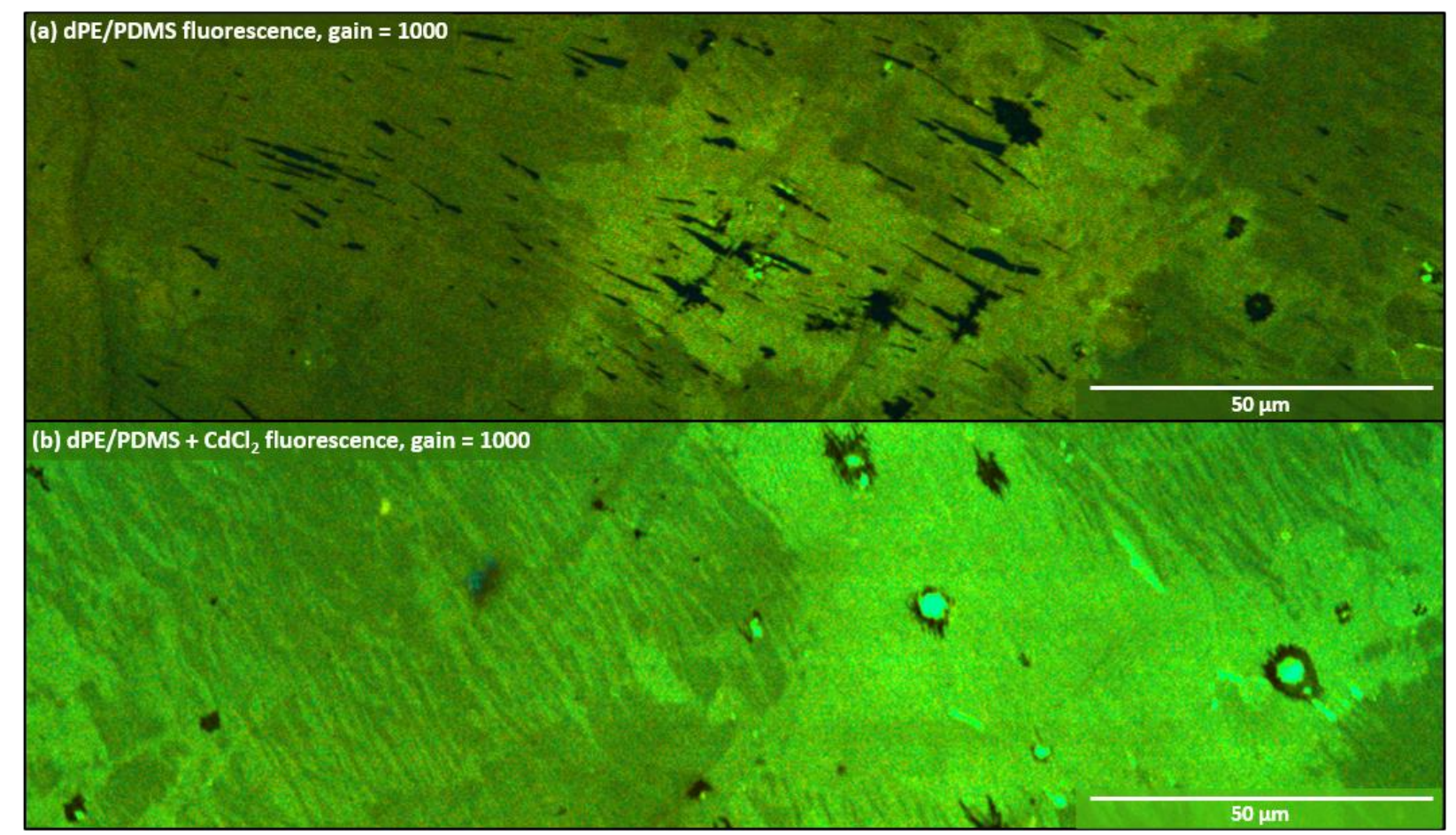

Figure 25. Fluorescence micrographs of dPE/PDMS (a) under standard imaging conditions, and (b) after exposure to $100 \mathrm{mM} \mathrm{CdCl}_{2}$ buffer. 


\section{Ultranarrow gold nanowire (AuNW) assembly on dPE.}

Previously, we have observed that striped phases of dPE on HOPG induce adsorption and ordering of ultranarrow gold nanowires (AuNWs). ${ }^{20}$ The nanowires we utilize in these experiments are grown using a modification of previously reported literature protocols, ${ }^{9-12}$ described in more detail in the Experimental Methods section. Briefly, the wires are grown in cyclohexane with a significant excess of oleylamine ligand, producing wires with Au core diameters from 1.5-2 nm, and lengths that can be controlled based on synthetic conditions in a range of $<100 \mathrm{~nm}$ to $>1 \mu \mathrm{m}$. In solution, the AuNWs form bundles due to depletion attraction interactions.

Our previous experiments, ${ }^{20}$ summarized in cartoon form in Supplementary Fig. 26, indicate that, when AuNW growth solution (containing excess oleylamine, and ions that are byproducts of reduction of $\mathrm{HAuCl}_{4}$ ), is exposed to dPE striped phases on HOPG, a complex assembly process occurs. Hemicylindrical micelles of OM form around rows of environmentally hydrated $\mathrm{dPE}$ headgroups, through adsorption of excess OM from the AuNW growth solution. These micelles protect the water adsorbed around the headgroups, forming an $\sim 1-\mathrm{nm}$-wide channel in which dPE headgroup dipoles can collectively reorient. Molecular dynamics simulations suggest a substantial dipole component normal to the surface. When polarizable AuNWs are exposed to these dipole arrays, it appears that the nanowires initially undergo transverse polarization, creating an attractive interaction between the wire and the dipole array on the HOPG, as well as repulsive interactions between adjacent wires on the surface, inducing separation and straightening of the wires. Assembly occurs in cyclohexane $(\varepsilon \sim 2)$, a low dielectric solvent for which the Bjerrum length (distance through which two elementary charges interact with a magnitude equal to kT) is $\sim 28$ $\mathrm{nm} .{ }^{21}$ Thus, repulsive interactions between wires are possible over substantial distances.

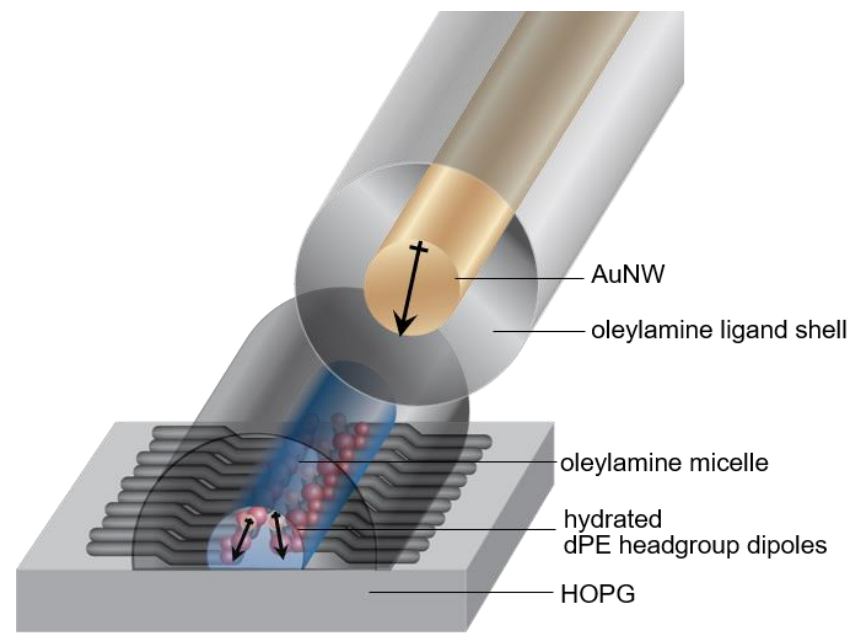

normal transverse polarization -> repulsion between AuNWs

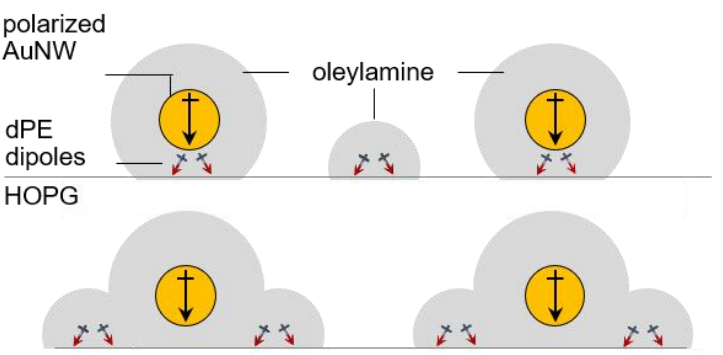

Figure 26. (left) Cartoon representation of polarization of AuNW near dPE headgroup dipole array on HOPG. (right) Cartoon representation of transverse polarization of multiple AuNWs leading to repulsion between wires.

Supplementary Fig. 27a illustrates adsorption of bundled AuNWs on bare HOPG, driven by alkyl-m interactions between oleylamine ligands and the HOPG basal plane; panel (b) in the same figure illustrates the adsorption and orientation behavior observed on striped phases of dPE on HOPG. When AuNW growth solution is exposed to bare PDMS, we observe minimal AuNW adsorption (Supplementary Fig. 27c), which is reasonable given the more heterogeneous surface structure and lack of possible alkyl- $\pi$ interactions with the substrate. In contrast, dPE/PDMS produces adsorption and separation of AuNWs (Supplementary Fig. 27d), consistent with elements of the behavior we observe for AuNWs on dPE/HOPG. We note that AuNWs on dPE/PDMS are somewhat less straight than those on dPE/HOPG, possibly consistent with relaxation of the striped phase surface on PDMS as shown in Fig. $3 a$, or with defects in the dPE layer structure induced by transfer to the PDMS. 

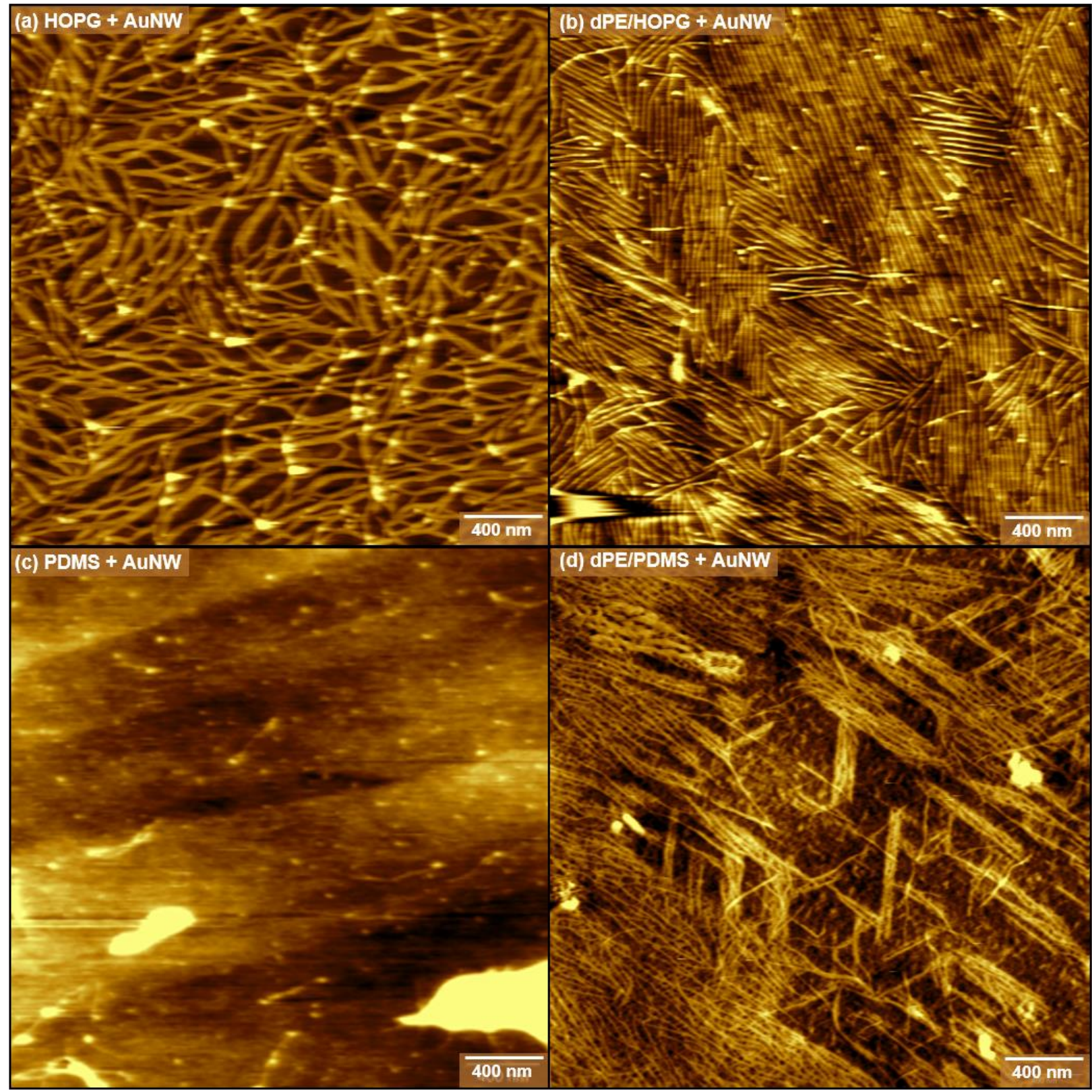

Figure 27. AuNW growth solution exposed to: (a) bare HOPG, producing bundled AuNW adsorption; (b) dPE/HOPG, producing adsorption, separation, and straightening, to form arrays of straight AuNWs; (c) bare PDMS, producing limited adsorption; and (d) dPE/PDMS, producing arrays of aligned, predominantly straight wires oriented with hexagonal symmetry.

\section{Functionalization of $\mathrm{dPE} / \mathrm{PDMS}$ and PCD-NH$/$ /PDMS with Rhodamine Red}

In the main manuscript, we use $\mu \mathrm{CP}-\mathrm{PCD}-\mathrm{NH}_{2}$ as a starting point for transfer of square patterns of striped phases to PDMS. Supplementary Fig. 28 shows a larger area of PCD- $\mathrm{NH}_{2} / \mathrm{PDMS}$ and was acquired in an area that illustrates that, while both patterning steps are relatively robust to the presence of individual step edges in the HOPG substrate, areas in which HOPG flakes away from the main substrate can impact transfer. 


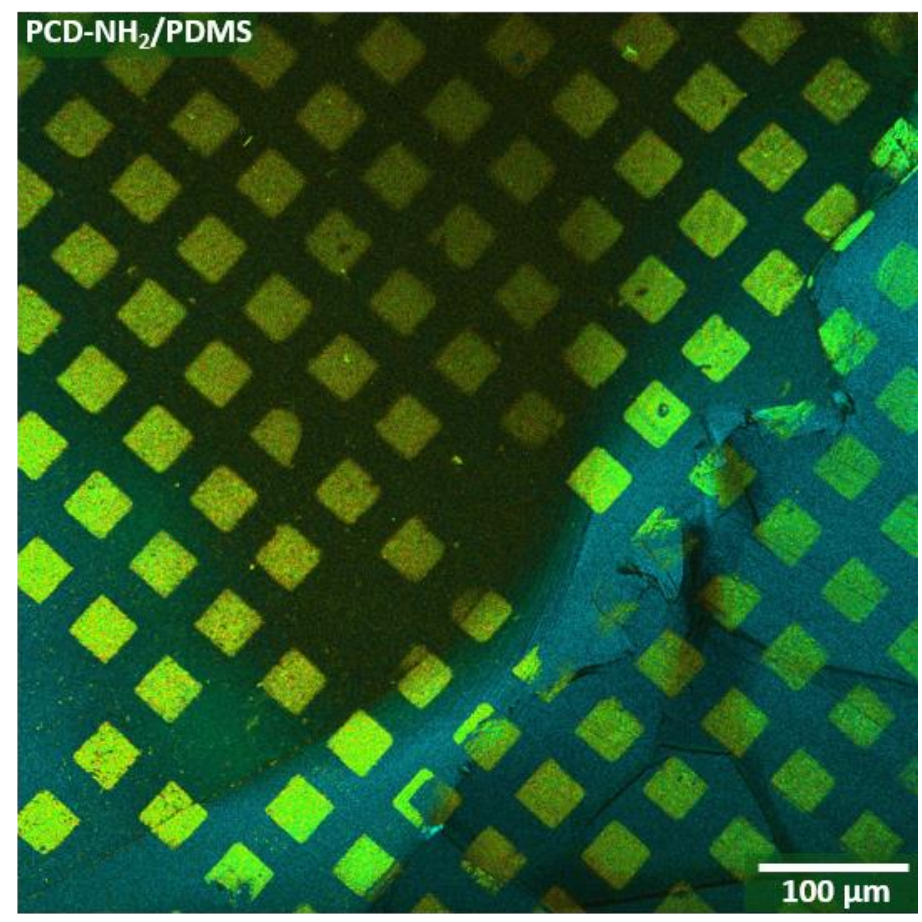

Figure 28. Fluorescence image of square pattern of PCD-NH $/$ PDMS transferred from an area surrounding a large HOPG step edge.

In the main manuscript, we expose striped monolayers of PCD-NH $\mathrm{N}_{2}$ on PDMS to an NHS ester of rhodamine red (RR) in order to functionalize the rows of primary amines in the headgroups. Here (Supplementary Fig. 29), we present results of similar experiments illustrating that the same process can be utilized on dPE/PDMS monolayer. Here, we show large SEM and fluorescence images of dPE on HOPG prior to transfer (Supplementary Fig. 29a,c) and after transfer to PDMS (Supplementary Fig. 29b,d). Supplementary Fig. 29e shows excitation and emission spectra of RR in solution. The main excitation spectrum (green trace) is acquired at $\lambda_{\mathrm{em}}=589 \mathrm{~nm}$; the main emission spectrum (red trace) is collected with $\lambda_{\mathrm{exc}}=$ $560 \mathrm{~nm}$. For comparison, we also show an emission spectrum (grey trace) collected at $\lambda_{\mathrm{exc}}=488 \mathrm{~nm}$, the excitation wavelength used for imaging PCDA and dPE in the manuscript. Supplementary Figs. 29f and $g$ show spectra collected from regions of interest in dPE films on PDMS after exposure to RR and extensive sonication in a mixture of solvents (see Experimental Methods) to remove physisorbed RR. When excited at $488 \mathrm{~nm}$ (the wavelength used to image dPE throughout the manuscript), emission is observed in the spectral region associated with RR (Supplementary Fig. 29f). Overall, the main emission peak appears broadened relative to the peak in solution. When the surface is imaged at an excitation wavelength (561 $\mathrm{nm})$ near the RR excitation peak, the emission spectrum (Supplementary Fig. 29g, blue trace) exhibits a strong peak with a maximum near $580 \mathrm{~nm}$. The blue spectrum shown in Supplementary Fig. $29 \mathrm{~g}$ was collected at $1 \%$ laser power, while PDA monolayers are typically imaged at $100 \%$ laser power throughout the manuscript. 


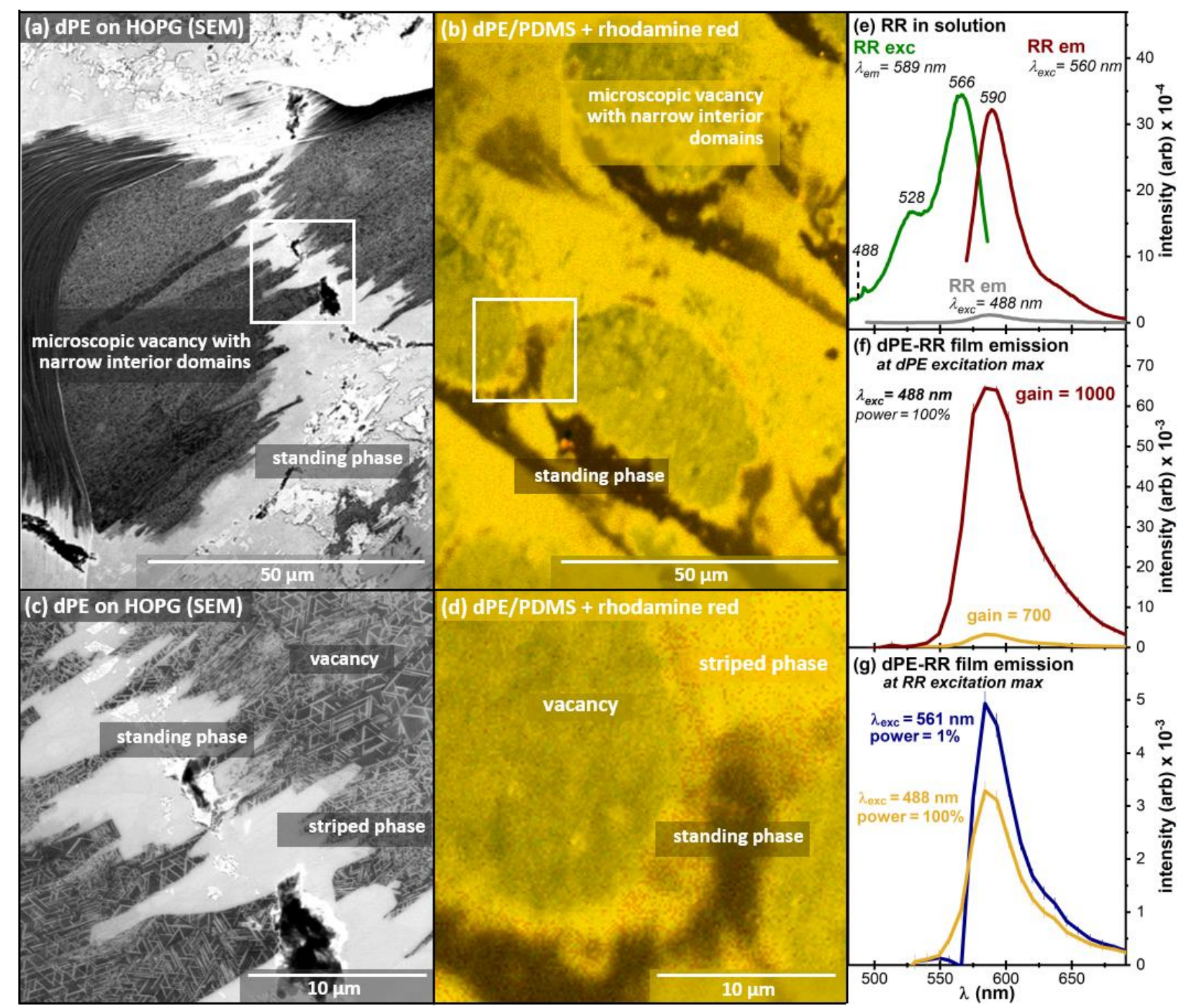

Figure 29. (a) SEM image of dPE/HOPG under transfer conditions utilized for RR reaction experiments. (b) Fluorescence emission image of dPE/PDMS after exposure to RR and cleaning by sonication in solvents as described in the Experimental Methods. (c,d) Insets from regions of $(\mathbf{a}, \mathbf{b})$ highlighted with white boxes. (e) Fluorescence excitation and emission spectra of RR. Excitation spectrum (green) is detected using $\lambda_{\mathrm{em}}=589 \mathrm{~nm}$. Emission spectrum (red) is detected using $\lambda_{\mathrm{exc}}=560 \mathrm{~nm}$; an emission spectrum (grey) was also collected using $\lambda_{\mathrm{exc}}=488 \mathrm{~nm}$. (f) $\mathrm{dPE} / \mathrm{PDMS}+\mathrm{RR}$ film emission spectra acquired at $\lambda_{\mathrm{exc}}=\mathbf{4 8 8} \mathrm{nm}$ (the wavelength utilized for polydiacetylene excitation throughout the manuscript) with detector gains of 700 (gold) and 1000 (red). (g) dPE/PDMS+RR film emission spectra acquired at standard RR excitation wavelength, $1 \%$ of the maximum laser power (blue) and at standard dPE excitation wavelength, at $100 \%$ of the maximum laser power. 


\section{Contact angle titrations.}

In the main manuscript, we present contact angle titrations of monolayers on HOPG. Here, we show a second titration curve acquired on PCDA/PDMS surfaces 46 days after transfer, illustrating that the contact angles are retained for 46 days.

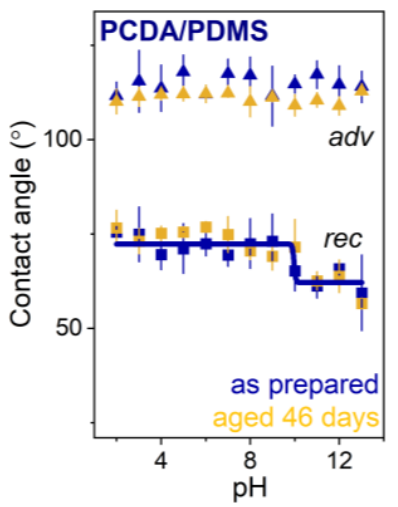

Figure 30. Contact angle titrations for PCDA/PDMS as originally prepared (blue) and after 46 days (gold).

\section{REFERENCES CITED}

1 Davis, T. C., Bang, J. J., Brooks, J. T., McMillan, D. G. \& Claridge, S. A. Hierarchical noncovalent functionalization of 2D materials by controlled Langmuir-Schaefer conversion. Langmuir 34, 1353-1362 (2018).

2 Thakar, R. \& Baker, L. A. Lithography-free production of stamps for microcontact printing of arrays. Anal. Meth. 2, 11801183 (2010).

3 Davis, T. C., Bechtold, J. O., Hayes, T. R., Villarreal, T. A. \& Claridge, S. A. Hierarchically patterned striped phases of phospholipids: Toward controlled presentation of carbohydrates. Faraday Discuss. 219, 229-243 (2019).

$4 \quad$ Hayes, T. R. et al. Multimicrometer noncovalent monolayer domains on layered materials through thermally controlled Langmuir-Schaefer conversion for noncovalent 2D functionalization. ACS Appl. Mater. Interf. 9, 36409-36416 (2017).

$5 \quad$ Walkowiak, J. et al. Pt-catalyzed hydrosilylation of 1,3-diynes with triorganosilanes: Regio- and stereoselective synthesis of mono- or bis-silylated adducts. J. Org. Chem. 84, 2358-2365 (2019).

6 Hamze, A., Provot, O., Alami, M. \& Brion, J. D. Platinum oxide catalyzed hydrosilylation of unsymmetrical internal aryl alkynes under ortho-substituent regiocontrol. Org. Lett. 7, 5625-5628 (2005).

7 Sabourault, N., Mignani, G., Wagner, A. \& Miokowski, C. Platinum oxide (PtO2): A potent hydrosilylation catalyst. Org. Lett. 4, 2117-2219 (2002).

8 Putzien, S., Louis, E. J., Nuyken, O. \& Kuhn, F. E. PtO2 as a "self-dosing" hydrosilylation catalyst. Catal. Sci. Technol. 2 , 725-729 (2012).

$9 \quad$ Halder, A. \& Ravishankar, N. Ultrafine single-crystalline gold nanowire arrays by oriented attachment. Adv. Mater. 19, 1854-1858 (2007).

10 Wang, C., Hu, Y. J., Lieber, C. M. \& Sun, S. H. Ultrathin Au nanowires and their transport properties. J. Am. Chem. Soc. 130, 8902-8903 (2008).

11 Huo, Z. Y., Tsung, C. K., Huang, W. Y., Zhang, X. F. \& Yang, P. D. Sub-two nanometer single crystal Au nanowires. Nano Lett. 8, 2041-2044 (2008).

12 Reiser, B. et al. Multivalent bonds in self-assembled bundles of ultrathin gold nanowires. Phys. Chem. Chem. Phys. 18, 27165-27169 (2016).

13 Lee, J. P., Hwang, H., Chae, S. \& Kim, J.-M. A reversibly mechanochromic conjugated polymer. Chem. Commun. 55, 9395-9398 (2019).

14 Pumtang, S., Siripornnoppakhun, W., Sukwattanasinitt, M. \& Ajavakom, A. Solvent colorimetric paper-based polydiacetylene sensors from diacetylene lipids. J. Colloid Interface Sci. 364, 366-372 (2011).

15 Howarth, N. M., Lindsell, W. E., Murray, E. \& Preston, P. N. Lipophilic peptide nucleic acids containing a 1,3-diyne function: Synthesis, characterization and production of derived polydiacetylene liposomes. Tetrahedron 61, 8875-8887 (2005).

16 Schwartz, J. J., Hohman, J. N., Morin, E. I. \& Weiss, P. S. Molecular flux dependence of chemical patterning by microcontact printing. ACS Appl. Mater. Interf. 5, 10310-10316 (2013).

17 Nečas, D. \& Klapetek, P. Gwyddion: An open-source software for SPM data analysis. Cent. Eur. J. Phys. 10, 181-188 (2012).

18 Chance, R. R., Patel, G. N., Turi, E. A. \& Khanna, Y. P. Energetics of thermal polymerization of a diacetylene crystal. J. Am. Chem. Soc. 100, 1307-1309 (1978).

19 Davis, T. C., Russell, S. R. \& Claridge, S. A. Edge-on adsorption of multi-chain functional alkanes stabilizes noncovalent monolayers on $\mathrm{MoS}_{2}$. Chem. Commun. 54, 11709-11712 (2018).

20 Porter, A. G. et al. 1-nm-wide hydrated dipole arrays regulate AuNW assembly on striped monolayers in nonpolar solvent. Chem 5, 2264-2275 (2019). 
Hsu, M. F., Dufresne, E. R. \& Weitz, D. A. Reverse micelles enable strong electrostatic interactions between colloidal particles in nonpolar solvents. Langmuir 21, 4881-4887 (2005). 